\title{
MULTISTATIONARITY IN STRUCTURED REACTION NETWORKS
}

\author{
ALICIA DICKENSTEIN, MERCEDES PÉREZ MILLÁN, ANNE SHIU, AND XIAOXIAN TANG
}

\begin{abstract}
Many dynamical systems arising in biology and other areas exhibit multistationarity (two or more positive steady states with the same conserved quantities). Although deciding multistationarity for a polynomial dynamical system is an effective question in real algebraic geometry, it is in general difficult to determine whether a given network can give rise to a multistationary system, and if so, to identify witnesses to multistationarity, that is, specific parameter values for which the system exhibits multiple steady states. Here we investigate both problems. First, we build on work of Conradi, Feliu, Mincheva, and Wiuf, who showed that for certain reaction networks whose steady states admit a positive parametrization, multistationarity is characterized by whether a certain "critical function" changes sign. Here, we allow for more general parametrizations, which make it much easier to determine the existence of a sign change. This is particularly simple when the steady-state equations are linearly equivalent to binomials; we give necessary conditions for this to happen, which hold for many networks studied in the literature. We also give a sufficient condition for multistationarity of networks whose steady-state equations can be replaced by equivalent triangular-form equations. Finally, we present methods for finding witnesses to multistationarity, which we show work well for certain structured reaction networks, including those common to biological signaling pathways. Our work relies on results from degree theory, on the existence of explicit rational parametrizations of the steady states, and on the specialization of Gröbner bases.
\end{abstract}

Keywords: reaction network, mass-action kinetics, multistationarity, parametrization, binomial ideal, Brouwer degree, Gröbner basis

\section{INTRODUCTION}

An important problem in many applications is to determine whether a given dynamical system is multistationary, and if so to find witnesses to multistationarity (parameter values for which the system exhibits two or more steady states with the same conserved quantities). Here we resolve these problems for dynamical systems arising under mass-action kinetics from reaction networks with particular structure; for instance, biological signaling networks. Specifically, our main results are criteria for multistationarity and procedures for obtaining witnesses for networks having the following structure:

(A) networks that admit a rational parametrization of the steady states and where the resulting critical function changes sign (Theorem 3.12), and

(B) networks for which the steady-state equations are linearly equivalent to binomial equations (Theorems 4.6 and 5.3) or triangular-form equations (Theorem 6.5).

The critical function in (A) refers to the composition of the steady-state parametrization with the determinant of the Jacobian matrix. Theorem 3.12 therefore generalizes recent results, which rely on degree theory, due to Conradi, Feliu, Mincheva, and Wiuf [4]. Specifically, we consider more general

Date: January 23, 2019. 
steady-state parametrizations since we allow the rate-constant parameters to depend on the steadystate concentrations, and we replace the parameters with "effective parameters", which are usually fewer. We show that the resulting critical functions can be much simpler to analyze than those in [4]. In particular, for "linearly binomial networks" (see Definition 3.4), deciding multistationarity from the critical function can be done by inspection (Theorem 4.6). We give sufficient conditions for a "MESSI network" (see [35]) to be a linearly binomial network, and moreover this transformation of the steady-state equations is explicit (Theorem 5.3). Such networks include many biological signaling pathways. Additionally, we explain how critical functions are related to discriminants (Proposition 3.21), and give conditions that guarantee that triangular-form equations, as in (B) above, exist (Corollary 6.12). This last result relies on prior work on the specialization of Gröbner bases. Finally, we illustrate our results on a number of reaction networks arising in biology. Indeed, our results allow us to investigate multistationarity in biological networks systematically, where previously only ad-hoc methods could be applied.

Our results fit in the context of recent progress on the problems of deciding multistationarity (reviewed in [27]; see also [1, 11]), obtaining witnesses for multistationarity (e.g., [35, 36]), and characterizing parameter regions for multistationarity (e.g., [3, 4, 19, 24, 40, 45]). Indeed, we give new criteria for multistationarity and methods for witnesses, and also show that the multistationary parameter regions arising from degree theory that we describe are open sets and thus full-dimensional (Theorem 4.10).

As mentioned above, one of our criteria for multistationarity involves examining the determinant of the Jacobian matrix. The first such criterion (without composing with a steady-state parametrization) was given by Craciun and Feinberg [8] in the absence of conservation relations, and then was extended by many researchers (e.g., [1, 9, 32, 39, 46]). One version of such a result, a so-called injectivity criterion, says: If every term in the determinant of the Jacobian matrix has the same sign, then the network is not multistationary for any choice of parameters. Also, under some hypotheses, the converse holds [1, 4, 8, 13]; see also the recent paper [33. Here we prove analogous results, after using steady-state parametrizations.

Steady-state parametrizations have already been shown to be useful in analyzing reaction networks [4, 5, 30, 35, 36, 43, and we build on those prior works. Similarly, like many before us, we use degree theory to decide multistationarity (see e.g. [4, 10, 12, ) and develop theory attuned to networks with certain structure [13, 22, 35], including binomials [25, 32, 36].

Given that our work harnesses several techniques that have already been used for analyzing reaction networks - steady-state parametrizations, degree theory, and structured reaction networks - we emphasize that our final results rely on new techniques. Specifically, we introduce the notion of effective parameters (Definition 3.2 to simplify previous approaches for a common class of structured networks, and we use results on specializations of Gröbner bases [29].

Finally, our work is related to the following open question: If a network $G$ admits a positive steady state that is degenerate, does this guarantee that $G$ admits multiple positive steady states? (This question is related to the Nondegeneracy Conjecture [28.) One might hope that perturbing the parameters, i.e., rate constants and conservation-law values, would break apart the degenerate steady state into two or more steady states. Several prior results answered the above question, under some hypotheses, in the affirmative [4, 8, 13, 17, 41. Some of these results also yield procedures for generating a witness to multistationarity. Here, we add new results to this list in Theorems 3.12 , 4.6, and 6.5, see also Procedure 4.7 for linearly binomial networks. 
The outline of our work is as follows. In Section 2, we introduce mass-action kinetics systems and recall a well-known result about Newton polytopes. In Sections 3 and 4 , we consider networks that admit steady-state parametrizations. We show that for linearly binomial networks the effective parameters are recovered from the steady states and there are only $s$ of them, where $s$ is the number of species variables, which is a number typically much smaller than the number of parameters. We prove that multistationarity is guaranteed (by degree theory) when the critical function changes sign. In Section 5, we consider so-called MESSI networks (which describe Modifications of type Enzyme-Substrate or Swap with Intermediates) [35], which include many biological signaling networks. We give sufficient conditions for such networks to be linearly binomial, which generalize Example 2.1 (continued along the paper) and Example 4.8. In Section 6, we consider networks whose steady-state equations can be replaced by equivalent triangular-form equations. We give sufficient conditions for a degenerate steady state of such a network to break into multiple steady states and we show that triangular-form equations exist under general conditions. We end with a Discussion in Section 7. Finally, we include, in three Appendices, proofs of those results which require further background.

\section{BACKGROUND}

In this section, we introduce reaction networks and their mass-action kinetics systems (Section 2.1), and then recall a useful result pertaining to Newton polytopes (Section 2.2).

2.1. Reaction networks. Here we largely follow the notation of Conradi, Feliu, Mincheva, and Wiuf [4]. A reaction network $G$ consists of a set of $s$ species $\left\{X_{1}, X_{2}, \ldots, X_{s}\right\}$ and a set of $m$ reactions:

$$
\sum_{i=1}^{s} \alpha_{i j} X_{i} \rightarrow \sum_{i=1}^{s} \beta_{i j} X_{i}, \quad j=1,2, \ldots, m,
$$

where $\alpha_{i j}$ and $\beta_{i j}$ are non-negative integers. The stoichiometric matrix of $G$, denoted by $N$, is the $s \times m$ matrix with $(i, j)$-entry equal to $\beta_{i j}-\alpha_{i j}$. Let $i m(N)^{\perp}$ denote the orthogonal complement of the image of the stoichiometric matrix $N$, and let $d=s-\operatorname{rank}(N)$. A conservation-law matrix of $G$, denoted by $W$, is any row-reduced $d \times s$-matrix whose rows form a basis of $i m(N)^{\perp}$. If all entries of $W$ are nonnegative and every column of $W$ contains at least one nonzero entry, that is, every species occurs with a positive coefficient in at least one conservation law, then $G$ is conservative.

The concentrations of the species $X_{1}, X_{2}, \ldots, X_{s}$ are denoted by $x_{1}, x_{2}, \ldots, x_{s}$, respectively. The evolution of the concentrations with respect to time is given by a system of ordinary differential equations:

$$
\dot{x}=f(x):=N \cdot v(x),
$$

where $x=\left(x_{1}, x_{2}, \ldots, x_{s}\right)$ and $v: \mathbb{R}_{\geq 0}^{s} \rightarrow \mathbb{R}_{\geq 0}^{m}$ is a reaction rate function. This function, in the case of mass-action kinetics, is given by:

$$
v_{j}(x)=\kappa_{j} x_{1}^{\alpha_{1 j}} x_{2}^{\alpha_{2 j}} \cdots x_{s}^{\alpha_{s j}}, \quad j=1,2, \ldots, m,
$$

where $\kappa_{j} \in \mathbb{R}_{>0}$ is called a reaction rate constant. We can consider the reaction rate constants as parameters $\kappa=\left(\kappa_{1}, \ldots, \kappa_{m}\right)$ and view the polynomials $f_{\kappa, i} \in \mathbb{Q}[\kappa, x]$, for $i=1, \ldots, s$. For ease of notation we will sometimes simply write $f_{i}$ in place of $f_{\kappa, i}$, for $i=1, \ldots, s$.

Our system (1) satisfies $W \dot{x}=W f_{\kappa}(x)=0$, and both the positive orthant $\mathbb{R}_{>0}^{s}$ and its closure $\mathbb{R}_{\geq 0}$ are forward-invariant for the dynamics. Thus, a trajectory $x(t)$ beginning at a nonnegative 
vector $x(0)=x^{0} \in \mathbb{R}_{>0}^{s}$ remains, for all positive time, in the following stoichiometric compatibility class with respect to the total-constant vector $c:=W x^{0} \in \mathbb{R}_{>0}^{d}$ :

$$
\mathcal{S}_{c}:=\left\{x \in \mathbb{R}_{\geq 0}^{s} \mid W x=c\right\},
$$

that is, $\mathcal{S}_{c}$ is also forward-invariant with respect to the dynamics (1). A steady state of (1) is a nonnegative concentration vector $x^{*} \in \mathbb{R}_{>0}^{s}$ at which the ODEs (1) vanish: $f_{\kappa}\left(x^{*}\right)=f\left(x^{*}\right)=0$. We distinguish between positive steady states $x^{*} \in \mathbb{R}_{>0}^{s}$ and boundary steady states $x^{*} \in \mathbb{R}_{>0}^{s} \backslash \mathbb{R}_{>0}^{s}$.

In order to analyze steady states within a stoichiometric compatibility class, we use the conservation laws in place of linearly dependent steady-state equations, as follows. Recall that the conservation-law matrix $W$ is row-reduced. Let $I=\left\{i_{1}, \ldots, i_{d}\right\}$ be the indices of the first nonzero coordinate of the rows of $W$, and assume that $i_{1}<i_{2}<\cdots<i_{d}$. Define the function $f_{c, \kappa}: \mathbb{R}_{\geq 0}^{s} \rightarrow \mathbb{R}^{s}$ by

$$
f_{c, \kappa, i}=f_{c, \kappa}(x)_{i}= \begin{cases}f_{\kappa, i}(x) & \text { if } i \notin I, \\ (W x-c)_{k} & \text { if } i=i_{k} \in I .\end{cases}
$$

This particular choice is needed for the validity of Theorem 3.12 below. We refer to system (2) as the system (1) augmented by conservation laws. For a rate-constant vector $\kappa \in \mathbb{R}_{>0}^{m}$ and a total-constant vector $c \in \mathbb{R}_{>0}^{d}$, we say $x^{*} \in \mathbb{R}_{\geq 0}^{s}$ is a steady state of the network for $\kappa$ and $c$ if it is a root of the augmented system $f_{c, \kappa}\left(x^{*}\right)=0$. Such a steady state $x^{*}$ is nondegenerate if the Jacobian matrix of $f_{c, \kappa}$ at $x^{*}$ has full rank (namely, equal to $s$ ).

A multistationary network admits two or more positive steady states for some rate-constant vector $\kappa$ and total-constant vector $c$. Non-multistationary networks are monostationary.

Example 2.1 (Phosphorylation/dephosphorylation of two substrates). Consider the following network, which is from [4, $\S 6.1$ in Supplementary Information]:

$$
\begin{aligned}
& \mathrm{A}+\mathrm{K} \underset{\kappa_{2}}{\stackrel{\kappa_{1}}{\rightleftharpoons}} \mathrm{AK} \stackrel{\kappa_{3}}{\longrightarrow} \mathrm{A}_{p}+\mathrm{K}, \quad \mathrm{A}_{p}+\mathrm{F} \underset{\kappa_{5}}{\stackrel{\kappa_{4}}{\rightleftharpoons}} \mathrm{A}_{p} \mathrm{~F} \stackrel{\kappa_{6}}{\longrightarrow} \mathrm{A}+\mathrm{F}, \\
& \mathrm{B}+\mathrm{K} \underset{\kappa_{8}}{\stackrel{\kappa_{7}}{\rightleftharpoons}} \mathrm{BK} \stackrel{\kappa_{9}}{\longrightarrow} \mathrm{B}_{p}+\mathrm{K}, \quad \mathrm{B}_{p}+\mathrm{F} \underset{\kappa_{11}}{\stackrel{\kappa_{10}}{\rightleftharpoons}} \mathrm{B}_{p} \mathrm{~F} \stackrel{\kappa_{12}}{\longrightarrow} \mathrm{B}+\mathrm{F} .
\end{aligned}
$$

In this network, phosphorylation and dephosphorylation of two substrates $A$ and $B$ are catalyzed by a kinase $K$ and a phosphatase $F$, respectively.

Following [4], let

$$
\begin{aligned}
& X_{1}=\mathrm{K}, \quad X_{3}=\mathrm{A}, \quad X_{5}=\mathrm{B}, \quad X_{7}=\mathrm{AK}, \quad X_{9}=\mathrm{A}_{p} \mathrm{~F}, \\
& X_{2}=\mathrm{F}, \quad X_{4}=\mathrm{A}_{p}, \quad X_{6}=\mathrm{B}_{p}, \quad X_{8}=\mathrm{BK}, \quad X_{10}=\mathrm{B}_{p} \mathrm{~F} .
\end{aligned}
$$

The system evolves according to the ODEs $\dot{x}=f_{\kappa}(x)$, where the function $f_{\kappa}=\left(f_{1}, \ldots, f_{10}\right)$ arising from mass-action kinetics is as follows:

$$
\begin{array}{ll}
f_{1}=-\kappa_{1} x_{1} x_{3}+\kappa_{2} x_{7}+\kappa_{3} x_{7}-\kappa_{7} x_{1} x_{5}+\kappa_{8} x_{8}+\kappa_{9} x_{8}, & \\
f_{2}=-\kappa_{4} x_{2} x_{4}+\kappa_{5} x_{9}+\kappa_{6} x_{9}-\kappa_{10} x_{2} x_{6}+\kappa_{11} x_{10}+\kappa_{12} x_{10}, & \\
f_{3}=-\kappa_{1} x_{1} x_{3}+\kappa_{2} x_{7}+\kappa_{6} x_{9}, & f_{4}=-\kappa_{4} x_{2} x_{4}+\kappa_{3} x_{7}+\kappa_{5} x_{9}, \\
f_{5}=-\kappa_{7} x_{1} x_{5}+\kappa_{8} x_{8}+\kappa_{12} x_{10}, & f_{6}=-\kappa_{10} x_{2} x_{6}+\kappa_{9} x_{8}+\kappa_{11} x_{10}, \\
f_{7}=\kappa_{1} x_{1} x_{3}-\kappa_{2} x_{7}-\kappa_{3} x_{7}, & f_{8}=\kappa_{7} x_{1} x_{5}-\kappa_{8} x_{8}-\kappa_{9} x_{8}, \\
f_{9}=\kappa_{4} x_{2} x_{4}-\kappa_{5} x_{9}-\kappa_{6} x_{9}, & f_{10}=\kappa_{10} x_{2} x_{6}-\kappa_{11} x_{10}-\kappa_{12} x_{10} .
\end{array}
$$


Letting $c_{1}, c_{2}, c_{3}, c_{4}$ denote the total amounts of $K, F, A$, and $B$, respectively, then the conservation laws are:

$x_{1}+x_{7}+x_{8}=c_{1}, \quad x_{2}+x_{9}+x_{10}=c_{2}, \quad x_{3}+x_{4}+x_{7}+x_{9}=c_{3}, \quad x_{5}+x_{6}+x_{8}+x_{10}=c_{4}$.

Every $x_{i}$ appears in at least one conservation law and all have nonnegative coefficients, so the network is conservative. The resulting conservation-law matrix $W$ is:

$$
\left(\begin{array}{llllllllll}
1 & 0 & 0 & 0 & 0 & 0 & 1 & 1 & 0 & 0 \\
0 & 1 & 0 & 0 & 0 & 0 & 0 & 0 & 1 & 1 \\
0 & 0 & 1 & 1 & 0 & 0 & 1 & 0 & 1 & 0 \\
0 & 0 & 0 & 0 & 1 & 1 & 0 & 1 & 0 & 1
\end{array}\right)
$$

which is already in row-reduced form, and the indices of the first nonzero coordinate of the rows are $1,2,3$, and 5 . So, by (2), the function $f_{c, \kappa}=f_{c, \kappa}(x)$ is as follows:

$$
\begin{array}{ll}
f_{c, \kappa, 1}=x_{1}+x_{7}+x_{8}-c_{1}, & f_{c, \kappa, 2}=x_{2}+x_{9}+x_{10}-c_{2}, \\
f_{c, \kappa, 3}=x_{3}+x_{4}+x_{7}+x_{9}-c_{3}, & f_{c, \kappa, 4}=-\kappa_{4} x_{2} x_{4}+\kappa_{3} x_{7}+\kappa_{5} x_{9}, \\
f_{c, \kappa, 5}=x_{5}+x_{6}+x_{8}+x_{10}-c_{4}, & f_{c, \kappa, 6}=-\kappa_{10} x_{2} x_{6}+\kappa_{9} x_{8}+\kappa_{11} x_{10}, \\
f_{c, \kappa, 7}=\kappa_{1} x_{1} x_{3}-\kappa_{2} x_{7}-\kappa_{3} x_{7}, & f_{c, \kappa, 8}=\kappa_{7} x_{1} x_{5}-\kappa_{8} x_{8}-\kappa_{9} x_{8}, \\
f_{c, \kappa, 9}=\kappa_{4} x_{2} x_{4}-\kappa_{5} x_{9}-\kappa_{6} x_{9}, & f_{c, \kappa, 10}=\kappa_{10} x_{2} x_{6}-\kappa_{11} x_{10}-\kappa_{12} x_{10} .
\end{array}
$$

2.2. Newton polytopes. Consider a real, multivariate polynomial

$$
f=b_{1} x^{\sigma_{1}}+b_{2} x^{\sigma_{2}}+\cdots+b_{\ell} x^{\sigma_{\ell}} \in \mathbb{R}\left[x_{1}, x_{2}, \ldots, x_{s}\right],
$$

where $\sigma_{i} \in \mathbb{Z}^{s}$ are distinct, $x=\left(x_{1}, x_{2}, \ldots, x_{s}\right), x^{\sigma}=\prod_{j=1}^{s} x_{j}^{\sigma_{i j}}$, and we have $b_{i} \neq 0$ for all $i$. The Newton polytope of $f$ is the convex hull of its exponent vectors:

$$
\mathrm{NP}(f):=\operatorname{conv}\left\{\sigma_{1}, \sigma_{2}, \ldots, \sigma_{\ell}\right\} \subseteq \mathbb{R}^{s} .
$$

We will use the following well-known lemma.

Lemma 2.2. For a real, multivariate polynomial $f$ as in (3), if $\sigma_{i}$ is a vertex of $N P(f)$, then there exists $x^{*} \in \mathbb{R}_{>0}^{s}$ such that $f\left(x^{*}\right)$ and $b_{i}$ have the same sign.

Indeed, as $\sigma_{i}$ is a vertex of $\mathrm{NP}(f)$, there exists an integer vector $\eta \in \mathbb{Z}^{s}$ such that the inner product $\eta \cdot x$ is maximized over $\mathrm{NP}(f)$ only at $\sigma_{i}$. Consider the univariate polynomial $g(\lambda)=f\left(\lambda^{\eta_{1}}, \ldots, \lambda^{\eta_{s}}\right)$. The degree of $g$ equals $\eta \cdot \sigma_{i}$ and thus it is clear that when $\lambda$ tends to $+\infty$, the sign of $g$ agrees with the sign of its leading coefficient $b_{i}$. This result is not true for non-vertex exponents, as the univariate polynomial $\lambda^{2}-2 \lambda+1$ shows.

\section{Establishing MUltistationarity USING DEGREE THEORY}

In this section we show that determining whether certain networks are multistationary is equivalent to checking whether their "critical functions" change sign (Theorem 3.12). Such critical functions arise from steady-state parametrizations (Definition 3.6), which generalize those considered by Conradi, Feliu, Mincheva, and Wiuf [4]. Another way we generalize the approach in [4] is by working in terms of certain "effective parameters" rather than the original rate constants. Accordingly, our statements extend results in [4] and our proofs rely heavily on their arguments (which use degree theory). We show in Examples 3.18 and 3.19 , that our critical functions can be simpler and easier to analyze than those from [4]. 
3.1. Parametrizations and critical functions. We will consider simplified versions of $f_{c, \kappa}$ obtained by linear operations. To motivate these "simplified versions", consider from Example 2.1 one coordinate of $f_{c, \kappa}(x)$ :

$$
f_{7}=\kappa_{1} x_{1} x_{3}-\left(\kappa_{2}+\kappa_{3}\right) x_{7} .
$$

We will replace $f_{7}$ by $\frac{1}{\kappa_{1}} f_{7}=x_{1} x_{3}-\left(\frac{\kappa_{2}+\kappa_{3}}{\kappa_{1}}\right) x_{7}$, and then view $a_{1}=\frac{\kappa_{2}+\kappa_{3}}{\kappa_{1}}$ as a new "effective parameter". So we will consider the rational function $a_{1}=a_{1}(\kappa)$ instead of the three original parameters $\kappa_{1}, \kappa_{2}, \kappa_{3}{ }^{1}$. We must require that any resulting reparametrization, which replaces the parameters $\kappa_{i}$, for $i=1, \ldots, m$, by new parameters $a_{j}$, for $j=1, \ldots, \bar{m}$, which are rational functions of them, is surjective (from $\mathbb{R}_{>0}^{m}$ to $\mathbb{R}_{>0}^{\bar{m}}$ ). This surjectivity implies that $\bar{m} \leq m$, and in fact the inequality $\bar{m}<m$ holds in Examples 3.19, 3.1, and 4.8. Indeed, one of our motivations for introducing effective parameters is to reduce the number of parameters and thereby simplify the system.

Next we present an important example to further motivate and clarify the notions of effective parameters and effective steady-state function (which will be defined in Definition 3.2). The network below in Example 3.1 underlies ERK regulation by dual-site phosphorylation by the kinase MEK (denoted by $E$ ) and dephosphorylation by the phosphatase MKP3 $(F)$. Rubinstein, Mattingly, Berezhkovskii, and Shvartsman showed that this network is multistationary, and found witnesses to multistationarity by sampling parameters [37. In this paper we take a more systematic approach to deciding multistationary and to finding a witness to multistationarity, via a simplified system $h_{c, a}(x)$ (see Definition 3.2 and Theorem 3.12 .

Example 3.1 (ERK network). Consider the following network from [37]:

$$
\begin{array}{cc}
\mathrm{S}_{00}+\mathrm{E} \underset{\kappa_{2}}{\stackrel{\kappa_{1}}{\rightleftharpoons}} \mathrm{S}_{00} \mathrm{E} \stackrel{\kappa_{3}}{\longrightarrow} \mathrm{S}_{01} \mathrm{E} \stackrel{\mathrm{k}_{\mathrm{cat}}}{\longrightarrow} \mathrm{S}_{11}+\mathrm{E}, & \mathrm{S}_{11}+\mathrm{F} \underset{\mathrm{l}_{2}}{\stackrel{\mathrm{l}_{1}}{\rightleftharpoons}} \mathrm{S}_{11} \mathrm{~F} \stackrel{\mathrm{l}_{3}}{\longrightarrow} \mathrm{S}_{10} \mathrm{~F} \stackrel{\mathrm{l}_{\text {cat }}}{\longrightarrow} \mathrm{S}_{00}+\mathrm{F}, \\
\mathrm{S}_{01} \mathrm{E} \underset{\mathrm{k}_{\mathrm{on}}}{\stackrel{\mathrm{k}_{\text {off }}}{\rightleftharpoons}} \mathrm{S}_{01}+\mathrm{E}, & \mathrm{S}_{10} \mathrm{~F} \underset{\mathrm{l}_{\mathrm{on}}}{\stackrel{\mathrm{l}_{\text {off }}}{\rightleftharpoons}} \mathrm{S}_{10}+\mathrm{F}, \\
\mathrm{S}_{10}+\mathrm{E} \underset{\mathrm{m}_{1}}{\stackrel{\mathrm{m}_{2}}{\rightleftharpoons}} \mathrm{S}_{10} \mathrm{E} \stackrel{\mathrm{m}_{3}}{\longrightarrow} \mathrm{S}_{11}+\mathrm{E}, & \mathrm{S}_{01}+\mathrm{F} \underset{\mathrm{n}_{2}}{\stackrel{\mathrm{n}_{1}}{\rightleftharpoons}} \mathrm{S}_{01} \mathrm{~F} \stackrel{\mathrm{n}_{3}}{\longrightarrow} \mathrm{S}_{00}+\mathrm{F} .
\end{array}
$$

The rate constants of the network are as follows:

$$
\kappa=\left(\kappa_{1}, \kappa_{2}, \kappa_{3}, k_{\text {cat }}, k_{\text {on }}, k_{\text {off }}, m_{1}, m_{2}, m_{3}, l_{1}, l_{2}, l_{3}, l_{\text {cat }}, l_{\text {on }}, l_{\text {off }}, n_{1}, n_{2}, n_{3}\right) \in \mathbb{R}_{>0}^{18} .
$$

Also, we have $s=12$ species:

$$
\begin{aligned}
& X_{1}=\mathrm{S}_{00}, \quad X_{3}=\mathrm{F}, \quad X_{5}=\mathrm{S}_{10} \mathrm{~F}, \quad X_{7}=\mathrm{S}_{01} \mathrm{E}, \quad X_{9}=\mathrm{S}_{01}, \quad X_{11}=\mathrm{S}_{00} \mathrm{E}, \\
& X_{2}=\mathrm{E}, \quad X_{4}=\mathrm{S}_{11} \mathrm{~F}, \quad X_{6}=\mathrm{S}_{01} \mathrm{~F}, \quad X_{8}=\mathrm{S}_{10} \mathrm{E}, \quad X_{10}=\mathrm{S}_{10}, \quad X_{12}=\mathrm{S}_{11} .
\end{aligned}
$$

From the $d=3$ conservation laws, which arise from the total amounts of substrate $S$, kinase $E$, and phosphatase $F$, respectively:

$$
\begin{aligned}
x_{1}+x_{4}+x_{5}+x_{6}+x_{7}+x_{8}+x_{9}+x_{10}+x_{11}+x_{12} & =c_{1}, \\
x_{2}+x_{7}+x_{8}+x_{11} & =c_{2}, \\
x_{3}+x_{4}+x_{5}+x_{6} & =c_{3},
\end{aligned}
$$

we obtain $f_{c, \kappa}(x)$ :

\footnotetext{
${ }^{1}$ In this example, $\frac{\kappa_{2}+\kappa_{3}}{\kappa_{1}}$ is what is referred to as a $K_{m}$-value in structural biochemistry.
} 


$$
\begin{array}{ll}
f_{c, \kappa, 1}=x_{1}+x_{4}+x_{5}+x_{6}+x_{7}+x_{8}+x_{9}+x_{10}+x_{11}+x_{12}-c_{1}, & f_{c, \kappa, 2}=x_{2}+x_{7}+x_{8}+x_{11}-c_{2}, \\
f_{c, \kappa, 3}=x_{3}+x_{4}+x_{5}+x_{6}-c_{3}, & f_{c, \kappa, 4}=l_{1} x_{3} x_{12}-l_{2} x_{4}-l_{3} x_{4}, \\
f_{c, \kappa, 5}=l_{o n} x_{3} x_{10}+l_{3} x_{4}-l_{c a t} x_{5}-l_{o f f} x_{5}, & f_{c, \kappa, 6}=n_{1} x_{3} x_{9}-n_{2} x_{6}-n_{3} x_{6}, \\
f_{c, \kappa, 7}=k_{o n} x_{2} x_{9}+\kappa_{3} x_{11}-k_{c a t} x_{7}-k_{o f f} x_{7}, & f_{c, \kappa, 8}=m_{2} x_{2} x_{10}-m_{1} x_{8}-m_{3} x_{8}, \\
f_{c, \kappa, 9}=-k_{o n} x_{2} x_{9}-n_{1} x_{3} x_{9}+k_{o f f} x_{7}+n_{2} x_{6}, & \\
f_{c, \kappa, 10}=-l_{o n} x_{3} x_{10}-m_{2} x_{2} x_{10}+m_{1} x_{8}+l_{o f f} x_{5}, & \\
f_{c, \kappa, 11}=\kappa_{1} x_{2} x_{1}-\kappa_{2} x_{11}-\kappa_{3} x_{11}, & \\
f_{c, \kappa, 12}=-l_{1} x_{3} x_{12}+k_{c a t} x_{7}+m_{3} x_{8}+l_{2} x_{4} . &
\end{array}
$$

We introduce the following choice of 13 effective parameters:

$$
\begin{aligned}
& \bar{a}_{1}=\frac{l_{c a t}}{k_{c a t}}, \quad \bar{a}_{2}=\frac{m_{3}}{l_{c a t}}, \quad \bar{a}_{3}=\frac{l_{3}}{l_{c a t}}, \quad \bar{a}_{4}=\frac{n_{3}}{k_{c a t}}, \quad \bar{a}_{5}=\frac{\kappa_{3}}{k_{c a t}}, \quad \bar{a}_{6}=\frac{m_{3}}{l_{\text {can }}}, \quad \bar{a}_{7}=\frac{l_{\text {off }}}{l_{\text {on }}}, \\
& \bar{a}_{8}=\frac{n_{3}}{k_{o n}}, \quad \bar{a}_{9}=\frac{k_{o f f}}{k_{o n}}, \quad \bar{a}_{10}=\frac{\kappa_{1}}{\kappa_{2}+\kappa_{3}}, \quad \bar{a}_{11}=\frac{m_{2}}{m_{1}+m_{3}}, \quad \bar{a}_{12}=\frac{l_{1}}{l_{2}+l_{3}}, \quad \bar{a}_{13}=\frac{n_{1}}{n_{2}+n_{3}} .
\end{aligned}
$$

Note that the resulting map $\bar{a}: \mathbb{R}_{>0}^{18} \rightarrow \mathbb{R}_{>0}^{13}$ given by $\kappa \mapsto \bar{a}(\kappa)$ is surjective.

Definition 3.2. Let $G$ be a network with $m$ reactions and $s$ species and let $\dot{x}=f_{\kappa}(x)$ denote the resulting mass-action system. Denote by $W$ a row-reduced conservation-law matrix and by $I$ the set of indices of the first nonzero coordinates of its rows, as in 2.1 . Enumerate the complement of $I$ :

$$
[s] \backslash I=\left\{j_{1}<j_{2}<\cdots<j_{s-d}\right\} .
$$

We say that $\bar{a}_{1}(\kappa), \bar{a}_{2}(\kappa), \ldots, \bar{a}_{\bar{m}}(\kappa) \in \mathbb{Q}(\kappa)$ form a set of effective parameters for $G$ if the following hold:

(i) $\bar{a}_{i}\left(\kappa^{*}\right)$ is defined for all $\kappa^{*} \in \mathbb{R}_{>0}^{m}$ and, moreover, $\bar{a}_{i}\left(\kappa^{*}\right)>0$ for every $i=1,2, \ldots, \bar{m}$,

(ii) the following reparametrization map is surjective:

$$
\begin{aligned}
\bar{a}: \mathbb{R}_{>0}^{m} & \rightarrow \mathbb{R}_{>0}^{\bar{m}} \\
\kappa & \mapsto\left(\bar{a}_{1}(\kappa), \bar{a}_{2}(\kappa), \ldots, \bar{a}_{\bar{m}}(\kappa)\right),
\end{aligned}
$$

(iii) there exists an $(s-d) \times(s-d)$ matrix $M(\kappa)$ with entries in $\mathbb{Q}(\kappa):=\mathbb{Q}\left(\kappa_{1}, \kappa_{2}, \ldots, \kappa_{m}\right)$ such that:

(a) for all $\kappa^{*} \in \mathbb{R}_{>0}^{m}$, the matrix $M\left(\kappa^{*}\right)$ is defined and, moreover, $\operatorname{det} M\left(\kappa^{*}\right)>0$, and

(b) letting $\left(\bar{h}_{j_{\ell}}\right)$ denote the functions obtained from $\left(f_{j_{\ell}}\right)$ via the linear operations defined by $M(\kappa)$, as follows:

$$
\left(\bar{h}_{j_{1}}, \bar{h}_{j_{2}}, \ldots, \bar{h}_{j_{s-d}}\right)^{\top}:=\quad M(\kappa)\left(f_{j_{1}}, f_{j_{2}}, \ldots, f_{j_{s-d}}\right)^{\top},
$$

every nonconstant coefficient in every $\bar{h}_{j_{\ell}}$ is equal to a rational-number multiple of some $\bar{a}_{i}(\kappa)$. In particular, $\bar{h}_{j_{\ell}}$ in $\mathbb{Q}(\kappa)[x]$.

Given such an effective parametrization, we consider for $\ell=1,2, \ldots, s-d$, polynomials $h_{j_{\ell}}=$ $h_{j_{\ell}}(a, x) \in \mathbb{Q}\left[a_{1}, a_{2}, \ldots, a_{\bar{m}}\right][x]$ (here, the $a_{i}$ 's are indeterminates) such that:

$$
\bar{h}_{j_{\ell}}=\left.h_{j_{\ell}}\right|_{a_{1}=\bar{a}_{1}(\kappa), \ldots, a_{\bar{m}}=\bar{a}_{\bar{m}}(\kappa)} \text {. }
$$


For $i=1,2, \ldots, s$ and any choice of $c \in \mathbb{R}_{>0}^{d}$ and $a \in \mathbb{R}_{>0}^{\bar{m}}$, set

$$
h_{c, a, i}=h_{c, a}(x)_{i}:= \begin{cases}h_{i}(a, x) & \text { if } i \notin I \\ (W x-c)_{k} & \text { if } i=i_{k} \in I .\end{cases}
$$

We say that the function $h_{c, a}: \mathbb{R}_{>0}^{s} \rightarrow \mathbb{R}^{s}$ is an effective steady-state function of $G$.

Remark 3.3. The choice of effective parameters is not unique. In fact, we can simply choose the effective parameters to be the $\kappa_{i}$ 's (that is, $\bar{a}_{i}\left(\kappa^{*}\right):=\kappa_{i}^{*}$ for all $i$ ), but in our examples we will instead choose the nonconstant coefficients (or $\mathbb{Q}$-multiples of them) of the $\bar{h}_{j}$ 's. Indeed, there are usually fewer such coefficients than $\kappa_{i}$ 's (see e.g. Examples 3.19, 3.1, and 4.8). However, we do not have an algorithm for determining a best choice of effective parameters.

Definition 3.4. A network is linearly binomial if there exist binomials $\bar{h}_{j_{1}}, \bar{h}_{j_{2}}, \ldots, \bar{h}_{j_{s-d}}$ and a matrix $M(\kappa)$ as in Definition 3.2 , such that equality (8) holds.

The networks in Examples 3.19 and 4.8 are linearly binomial. We will abstract the features that imply this property in Theorem 5.3 .

Remark 3.5. Every linearly binomial network is a "binomial network" as defined by Sadeghimanesh and Feliu [38].

Definition 3.6. Let $G$ be a network with $m$ reactions, $s$ species, and row-reduced conservation-law matrix $W$. Let $f_{c, \kappa}$ arise from $G$ and $W$ as in (2). Suppose that $h_{c, a}$ is an effective steady-state function of $G$, as in (10), arising from a matrix $M(\kappa)$, as in (8), a reparametrization map $\bar{a}$, as in (7), and polynomials $h_{j_{\ell}}$ 's as in (9) as in Definition 3.2 .

We say that the positive steady states of $G$ admit a positive parametrization with respect to $h_{c, a}$ if there exists a function:

$$
\begin{aligned}
\phi: \mathbb{R}_{>0}^{\hat{m}} \times \mathbb{R}_{>0}^{\hat{s}} & \rightarrow \mathbb{R}_{>0}^{\bar{m}} \times \mathbb{R}_{>0}^{s} \\
(\hat{a}, \hat{x}) & \mapsto \phi(\hat{a}, \hat{x})
\end{aligned}
$$

for some $\hat{m} \leq \bar{m}$ and $\hat{s} \leq s$, such that:

(i) $\phi(\hat{a}, \hat{x})$ extends the vector $(\hat{a}, \hat{x})$. More precisely, there exists a natural projection $\pi$ : $\mathbb{R}_{>0}^{\bar{m}} \times \mathbb{R}_{>0}^{s} \rightarrow \mathbb{R}_{>0}^{\hat{m}} \times \mathbb{R}_{>0}^{\hat{s}}$ such that $\pi \circ \phi$ is equal to the identity map.

(ii) Consider any $(a, x) \in \mathbb{R}_{>0}^{\bar{m}} \times \mathbb{R}_{>0}^{s}$. Then, the equality $h_{i}(a, x)=0$ holds for every $i \notin I$ if and only if there exists $\left(\hat{a}^{*}, \hat{x}^{*}\right) \in \mathbb{R}_{>0}^{\hat{m}} \times \mathbb{R}_{>0}^{\hat{s}}$ such that $(a, x)=\phi\left(\hat{a}^{*}, \hat{x}^{*}\right)$.

Note that given any $(a, x) \in \mathbb{R}_{>0}^{\bar{m}} \times \mathbb{R}_{>0}^{s}$, with $(a, x)=\phi(\hat{a}, \hat{x})$, if we set

$$
c=W x,
$$

then $h_{c, a}(x)=0$, where $h_{c, a}$ is the effective steady state function in Definition 3.2 .

Moreover, we can summarize the information in Definition 3.6 by asking that the diagram below commutes, where the maps $\mu(\kappa, x):=(\bar{a}(\kappa), x)$ and $\phi$ are surjective:

$$
\begin{aligned}
&\left\{\left(\kappa^{*}, x^{*}\right) \in \mathbb{R}_{>0}^{m} \times \mathbb{R}_{>0}^{s}:\left.f_{i}\right|_{\kappa=\kappa^{*}, x=x^{*}}=0 \text { for all } i \notin I\right\} \\
& \mathbb{R}_{>0}^{\hat{m}} \times \mathbb{R}_{>0}^{\hat{s}} \stackrel{\phi}{\longrightarrow} \mathbb{R}_{>0}^{\bar{m}} \times \mathbb{R}_{>0}^{s}
\end{aligned}
$$


Example 3.7 (ERK network, continued). Let

$$
M(\kappa)=\left(\begin{array}{ccccccccc}
\frac{1}{l_{2}+l_{3}} & 0 & 0 & 0 & 0 & 0 & 0 & 0 & 0 \\
0 & \frac{1}{l_{c a t}} & 0 & 0 & \frac{1}{l_{c a t}} & 0 & \frac{1}{l_{c a t}} & 0 & 0 \\
0 & 0 & \frac{1}{n_{2}+n_{3}} & 0 & 0 & 0 & 0 & 0 & 0 \\
0 & 0 & \frac{1}{k_{c a t}} & \frac{1}{k_{c a t}} & 0 & \frac{1}{k_{c a t}} & 0 & 0 & 0 \\
0 & 0 & 0 & 0 & \frac{1}{m_{1}+m_{3}} & 0 & 0 & 0 & 0 \\
0 & 0 & \frac{1}{k_{o n}} & 0 & 0 & \frac{1}{k_{o n}} & 0 & 0 & 0 \\
0 & 0 & 0 & 0 & \frac{1}{l_{l n}} & 0 & \frac{1}{l_{o n}} & 0 & 0 \\
0 & 0 & 0 & 0 & 0 & 0 & 0 & \frac{1}{\kappa_{2}+\kappa_{3}} & 0 \\
\frac{1}{k_{c a t}} & \frac{1}{k_{c a t}} & 0 & 0 & \frac{1}{k_{c a t}} & 0 & \frac{1}{k_{c a t}} & 0 & \frac{1}{k_{c a t}}
\end{array}\right)
$$

It is straightforward to check that $\operatorname{det} M(\kappa)>0$ for all $\kappa \in \mathbb{R}_{>0}^{18}$. From the effective parameters (5) and equations (8)-(10), the resulting system $h_{c, a}(x)$ is:

$$
\begin{array}{ll}
h_{c, a, 1}=x_{1}+x_{4}+x_{5}+x_{6}+x_{7}+x_{8}+x_{9}+x_{10}+x_{11}+x_{12}-c_{1}, & h_{c, a, 2}=x_{2}+x_{7}+x_{8}+x_{11}-c_{2}, \\
h_{c, a, 3}=x_{3}+x_{4}+x_{5}+x_{6}-c_{3}, & h_{c, a, 4}=\underline{a_{12}} x_{3} x_{12}-x_{4}, \\
h_{c, a, 5}=\underline{a_{3}} x_{4}-x_{5}-a_{2} x_{8}, & h_{c, a, 6}=\underline{a_{13}} x_{3} x_{9}-x_{6}, \\
h_{c, a, 7}=\underline{a_{5}} x_{11}-a_{4} x_{6}-x_{7}, & h_{c, a, 8}=\underline{a_{11}} x_{2} x_{10}-x_{8} \\
h_{c, a, 9}=\underline{a_{9}} x_{7}-x_{2} x_{9}-a_{8} x_{6}, & h_{c, a, 10}=\underline{a_{7}} x_{5}-x_{3} x_{10}-a_{6} x_{8}, \\
h_{c, a, 11}=\bar{a}_{10} x_{1} x_{2}-x_{11}, & \\
h_{c, a, 12}=x_{7}-\underline{a_{1}} x_{5} . &
\end{array}
$$

Let $\hat{a}=\left(a_{2}, a_{4}, a_{6}, a_{8}\right)$ and $\hat{x}=x$. By solving the non-conservation-law equations $h_{c, a}(x)_{i}=0$ (for $i=4, \ldots, 12)$, for the underlined unknowns $a_{1}, a_{3}, a_{5}, a_{7}, a_{9}, a_{10}, a_{11}, a_{12}, a_{13}$, we obtain the positive parametrization $\phi: \mathbb{R}_{>0}^{16} \rightarrow \mathbb{R}_{>0}^{13} \times \mathbb{R}_{>0}^{12}$ with respect to the effective steady-state function $h_{c, a}$, where $\phi(\hat{a}, \hat{x})\left(=\phi\left(a_{2}, a_{4}, a_{6}, a_{8} ; x\right)\right)$ is defined as

$$
\left(\frac{x_{7}}{x_{5}}, a_{2}, \frac{a_{2} x_{8}+x_{5}}{x_{4}}, a_{4}, \frac{a_{4} x_{6}+x_{7}}{x_{11}}, a_{6}, \frac{a_{6} x_{8}+x_{3} x_{10}}{x_{5}}, a_{8}, \frac{a_{8} x_{6}+x_{2} x_{9}}{x_{7}}, \frac{x_{11}}{x_{1} x_{2}}, \frac{x_{8}}{x_{2} x_{10}}, \frac{x_{4}}{x_{3} x_{12}}, \frac{x_{6}}{x_{3} x_{9}} ; x\right) .
$$

We will use this information in Example 3.18 below.

We need one more definition in order to state Theorem 3.12 .

Definition 3.8. Under the notation and hypotheses of Definition 3.6, assume that the steady states of $G$ admit a positive parametrization with respect to $h_{c, a}$. For such a positive parametrization $\phi$, the critical function $C: \mathbb{R}_{>0}^{\hat{m}} \times \mathbb{R}_{>0}^{\hat{s}} \rightarrow \mathbb{R}$ is given by:

$$
C(\hat{a}, \hat{x})=\left.\left(\operatorname{det} \operatorname{Jac}\left(h_{c, a}\right)\right)\right|_{(a, x)=\phi(\hat{a}, \hat{x})},
$$

where $\operatorname{Jac}\left(h_{c, a}\right)$ denotes the Jacobian matrix of $h_{c, a}$ with respect to $x$.

Remark 3.9. Assume $\left(a^{*}, x^{*}\right)=\phi\left(\hat{a}^{*}, \hat{x}^{*}\right)$. It follows that $C\left(\hat{a}^{*}, \hat{x}^{*}\right)=0$ if and only if $x^{*}$ is a degenerate steady state of any mass-action system defined by network $G$ for the total-constant vector $c^{*}=W x^{*}$ and a choice of rate-constant vector $\kappa^{*}$ for which $a^{*}=\bar{a}\left(\kappa^{*}\right)$. Here, $\bar{a}\left(\kappa^{*}\right)$ refers to the map (7).

Remark 3.10 (Comparison with [4]). The parametrizations considered by Conradi, Feliu, Mincheva, and Wiuf did not allow the reaction rates $\kappa_{i}$ to depend on the $x_{i}$ 's [4]. Specifically, their parametrizations have the form $(\kappa, \hat{x}) \mapsto(\kappa,(\hat{x}, \Phi(\kappa, \hat{x}))$. The resulting critical functions are 
denoted by " $a(\hat{x})$ " in their work (notice that " $a(\hat{x})$ " also depends on $\kappa$, and the " $a$ " is a different notion from our $a$ in $h_{c, a}$ ); see Examples 3.18 and 3.19 . Additionally, the critical functions in [4] simply arise from the case when the matrix $M(\kappa)$ in (8) is the identity matrix (and the "reparametrization" map $\bar{a}$, in (7), is the identity map). In summary, we allow for more general positive parametrizations and critical functions than in [4], and hence Theorem 3.12 below generalizes [4, Theorem 1]; our proof is just a translation of their arguments to our setting.

Remark 3.11 (Existence of steady-state parametrizations). Steady-state parametrizations exist for many biological signaling networks [16, 35, 43. They can be computed by following one of the procedures in the references above, or, as suggested in [4, 5], by using computer-algebra software to solve the steady-state equations for all but $d$ variables (see, e.g., Example 3.19), eliminating if possible all intermediates. (Here $d$ is the number of conservation laws.)

A network is dissipative if for all choices of rate constants and stoichiometric compatibility classes $\mathcal{S}_{c}$, there exists a compact subset of $\mathcal{S}_{c}$ which every trajectory beginning in $\mathcal{S}_{c}$ eventually enters. Every conservative network is dissipative [4, pg. 6].

Theorem 3.12. Under the notation and hypotheses of Definitions 3.6 and 3.8, assume also that $G$ is a dissipative network without boundary steady states in any compatibility class.

(A) Multistationarity. $G$ is multistationary if there exists $\left(\hat{a}^{*}, \hat{x}^{*}\right) \in \mathbb{R}_{>0}^{\hat{m}} \times \mathbb{R}_{>0}^{\hat{s}}$ such that

$$
\operatorname{sign}\left(C\left(\hat{a}^{*}, \hat{x}^{*}\right)\right)=(-1)^{\operatorname{rank}(N)+1},
$$

where $N$ denotes the stoichiometric matrix of $G$.

(B) Witness to multistationarity. Every $\left(\hat{a}^{*}, \hat{x}^{*}\right) \in \mathbb{R}_{>0}^{\hat{m}} \times \mathbb{R}_{>0}^{\hat{s}}$ with $\operatorname{sign}\left(C\left(\hat{a}^{*}, \hat{x}^{*}\right)\right)=$ $(-1)^{\operatorname{rank}(N)+1}$ yields a witness to multistationarity $\left(\kappa^{*}, c^{*}\right)$ as follows. Let $\left(a^{*}, x^{*}\right)=$ $\phi\left(\hat{a}^{*}, \hat{x}^{*}\right)$. Let $c^{*}=W x^{*}$ (so, $c^{*}$ is the total-constant vector defined by $x^{*}$, where $W$ is the conservation-law matrix), and let $\kappa^{*} \in \mathbb{R}_{>0}^{m}$ be such that $\bar{a}\left(\kappa^{*}\right)=a^{*}$.

(C) Monostationarity. $G$ is monostationary if $\operatorname{sign}(C(\hat{a}, \hat{x}))=(-1)^{\operatorname{rank}(N)}$ for all $\left(\hat{a}^{*}, \hat{x}^{*}\right) \in$ $\mathbb{R}_{>0}^{\hat{m}} \times \mathbb{R}_{>0}^{\hat{s}}$.

Proof. We begin with (A) and (B). Assume that $\left(\hat{a}^{*}, \hat{x}^{*}\right) \in \mathbb{R}_{>0}^{\hat{m}} \times \mathbb{R}_{>0}^{\hat{s}} \operatorname{satisfies} \operatorname{sign}\left(C\left(\hat{a}^{*}, \hat{x}^{*}\right)\right)=$ $(-1)^{\operatorname{rank}(N)+1}$. That is, $\left.\operatorname{sign}\left(\operatorname{det} \operatorname{Jac}\left(h_{c, a}\right)\right)\right|_{(a, x)=\phi\left(\hat{a}^{*}, \hat{x}^{*}\right)}=(-1)^{\operatorname{rank}(N)+1}$.

By [4, Theorem 1], $G$ is multistationary if there exist $\kappa^{*} \in \mathbb{R}_{>0}^{m}$ and $x^{*} \in \mathbb{R}_{>0}^{s}$ such that

(i) $\left.f_{i}\right|_{\kappa=\kappa^{*}, x=x^{*}}=0$ for all $i \notin I$, and

(ii) $\left.\operatorname{sign}\left(\operatorname{det} \operatorname{Jac}\left(f_{c, \kappa}\right)\right)\right|_{\kappa=\kappa^{*}, x=x^{*}}=(-1)^{\operatorname{rank}(N)+1}$.

Moreover, in this case, [4, Theorem 1] gives the following witness to multistationarity: $c^{*}=W x^{*}$ and $\kappa^{*}$. To use this result, let $\left(a^{*}, x^{*}\right)=\phi\left(\hat{a}^{*}, \hat{x}^{*}\right)$, and then define $c^{*}=W x^{*}$ and pick $\kappa^{*} \in \mathbb{R}_{>0}^{m}$ such that $\bar{a}\left(\kappa^{*}\right)=a^{*}$. Then we only need to show that conditions (i) and (ii) above hold.

To see (i), recall that the positive-determinant matrix $M(\kappa)$ transforms the $f_{i}$ 's, for $i \notin I$, to the $\bar{h}_{i}$ 's, as in (8). So, we need to show that $\left.\bar{h}_{i}\right|_{x=x^{*}, \kappa=\kappa^{*}}=0$ if $i \notin I$. Indeed,

$$
\left.\bar{h}_{i}\right|_{x=x^{*}, \kappa=\kappa^{*}}=\left.h_{i}\right|_{x=x^{*}, a=\bar{a}\left(\kappa^{*}\right)}=\left.h_{i}\right|_{(a, x)=\phi\left(\hat{a}^{*}, \hat{x}^{*}\right)}=0,
$$

where the final equality comes from requirement (ii) for a positive parametrization (in Definition 3.6.). 
For (ii), we first note that (8) implies the following:

$$
\left.\left(h_{c, a, 1}, h_{c, a, 2}, \ldots, h_{c, a, s}\right)^{\top}\right|_{a=\bar{a}}=\widetilde{M}(\kappa)\left(f_{c, \kappa, 1}, f_{c, \kappa, 2}, \ldots, f_{c, \kappa, s}\right)^{\top},
$$

where $\widetilde{M}(\kappa)$ is the $(s \times s)$-matrix obtained from $M(\kappa)$, in (8), by inserting rows and columns corresponding to the indices $i_{1}, i_{2}, \ldots, i_{d}$ such that row $i_{k}$ and column $i_{k}$ are both the canonical basis vector $e_{i_{k}}$, for $k=1,2, \ldots, d$. Thus, $\operatorname{det} \widetilde{M}(\kappa)=\operatorname{det} M(\kappa)>0$ for any $\kappa \in \mathbb{R}_{>0}^{m}$, and so 12 ) yields:

$$
\begin{aligned}
\left.\operatorname{sign}\left(\operatorname{det} \operatorname{Jac}\left(f_{c, \kappa}\right)\right)\right|_{\kappa=\kappa^{*}, x=x^{*}} & =\left.\operatorname{sign}\left(\operatorname{det} \operatorname{Jac}\left(h_{c, a}\right)\right)\right|_{(a, x)=\left(\bar{a}\left(\kappa^{*}\right), x^{*}\right)=\phi\left(\hat{a}^{*}, \hat{x}^{*}\right)} \\
& =\operatorname{sign}\left(C\left(\hat{a}^{*}, \hat{x}^{*}\right)\right) \\
& =(-1)^{\operatorname{rank}(N)+1}
\end{aligned}
$$

where the final equality is by hypothesis. Thus, (ii) holds.

For $(\mathrm{C})$, assume that $\operatorname{sign}(C(\hat{a}, \hat{x}))=(-1)^{\operatorname{rank}(N)}$ for all $\left(\hat{a}^{*}, \hat{x}^{*}\right) \in \mathbb{R}_{>0}^{\hat{m}} \times \mathbb{R}_{>0}^{\hat{s}}$. Suppose that $\kappa^{*} \in \mathbb{R}_{>0}^{m}$ and $x^{*} \in \mathbb{R}_{>0}^{s}$ are such that $\left.f_{i}\right|_{\kappa=\kappa^{*}, x=x^{*}}=0$ for all $i \notin I$. By [4, Theorem 1], we only need to show the following:

$$
\left.\operatorname{sign}\left(\operatorname{det} \operatorname{Jac}\left(f_{c, \kappa}\right)\right)\right|_{\kappa=\kappa^{*}, x=x^{*}}=(-1)^{\operatorname{rank}(N)} .
$$

To this end, let $\left(\hat{a}^{*}, \hat{x}^{*}\right)=\pi \circ \mu\left(\kappa^{*}, x^{*}\right)$, so that (using the commutative diagram following Definition 3.6) we have $\phi\left(\hat{a}^{*}, \hat{x}^{*}\right)=\left(\bar{a}\left(\kappa^{*}\right), x^{*}\right)$. We now verify equality 13):

$$
\begin{aligned}
\left.\operatorname{sign}\left(\operatorname{det} \operatorname{Jac}\left(f_{c, \kappa}\right)\right)\right|_{\kappa=\kappa^{*}, x=x^{*}} & =\left.\operatorname{sign}\left(\operatorname{det} \operatorname{Jac}\left(h_{c, a}\right)\right)\right|_{(a, x)=\left(\bar{a}\left(\kappa^{*}\right), x^{*}\right)=\phi\left(\hat{a}^{*}, \hat{x}^{*}\right)} \\
& =\operatorname{sign}\left(C\left(\hat{a}^{*}, \hat{x}^{*}\right)\right) \\
& =(-1)^{\operatorname{rank}(N)} .
\end{aligned}
$$

Remark 3.13. Theorem 3.12(B) suggests a procedure for finding a witness to multistationarity, which relies on picking some $\left(\hat{a}^{*}, \hat{x}^{*}\right)$ with $\operatorname{sign}\left(C\left(\hat{a}^{*}, \hat{x}^{*}\right)\right)=(-1)^{\operatorname{rank}(N)+1}$. There is no general method for picking such a vector $\left(\hat{a}^{*}, \hat{x}^{*}\right)$, but we can sometimes accomplish this via the Newton polytope of $C(\hat{a}, \hat{x})$, namely, when one of the vertices corresponds to a coefficient of $C(\hat{a}, \hat{x})$ with the desired sign $(-1)^{\operatorname{rank}(N)+1}$ (recall Lemma 2.2). Indeed, we will see in Section 4 that this approach always succeeds for linearly binomial networks.

Remark 3.14. Theorem 3.12 can guarantee multiple steady states, but does not say anything about whether they are stable [4]. Also in that theorem, "monostationarity" in part (C) can be strengthened to state the existence of a unique positive steady state in each compatibility class [4, Theorem 1].

Remark 3.15. We use $M(\kappa)$ to denote, as in (8), the matrix transforming the $f_{i}$ 's to the $\bar{h}_{i}$ 's, while the authors of [4] use " $M(x)$ " to denote the matrix $\operatorname{Jac}\left(f_{c, \kappa}\right)$, after substituting the parametrization.

Remark 3.16. Our strategy for constructing a useful matrix $M(\kappa)$ as in (8), is to perform linear operations on the $f_{i}(x)$ 's to obtain as many binomials as possible (see, e.g., Example 3.1). Additionally, we will show in Theorem 5.3 that for many biological signaling networks, a suitable $M(\kappa)$ exists so that in the resulting system $h_{c, a}$, every non-conservation-law equation is a binomial. 
Remark 3.17. Theorem 3.12 relies on degree theory, which is why two key hypotheses are required: being dissipative and having no boundary steady states. A discussion on how to verify these two hypotheses is in [4, pp. 11-12]. In the examples below, the networks are conservative and thus dissipative, and checking that there are no boundary steady states can be done using, for instance, results from [42] or [35, Theorem 3.13].

3.2. Examples. We present two examples. In the first one, we will see that the critical function arising from $h_{c, a}$ in Example 3.7 is simpler than that allowed by Conradi, Feliu, Mincheva, and Wiuf [4]. We will also see in this example how to use Theorem 3.12 to obtain a witness to multistationarity.

Example 3.18 (ERK network, continued). The critical function $C(\hat{a}, \hat{x})$ derived by the positive parametrization in Example 3.7 (recall that $\left.(\hat{a}, \hat{x})=\left(a_{2}, a_{4}, a_{6}, a_{8}, x\right)\right)$ is a rational function, where the denominator is the monomial $x_{1} x_{2} x_{3} x_{5} x_{9} x_{10} x_{12}$, and the numerator has total degree 11 and 704 terms.

This network is conservative (see (4)) and hence dissipative. It is straightforward to check (for instance, using criteria in [42]) that it has no boundary steady states. In fact, the ERK network is a MESSI network (see the definition in Section 5) and the absence of boundary steady states is a direct and easy consequence of Theorem $3.13 \mathrm{in} \mathrm{[35.} \mathrm{So} \mathrm{we} \mathrm{can} \mathrm{apply} \mathrm{Theorem} 3.12$ to find a witness to multistationarity. Accordingly, we compute $\operatorname{rank}(N)=s-d=12-3=9$, so the sign of interest is $(-1)^{\operatorname{rank}(N)+1}=1$. Thus, we must find $\left(\hat{a}^{*}, \hat{x}^{*}\right)$ for which $C\left(\hat{a}^{*}, \hat{x}^{*}\right)>0$. We find the following such point by using a vertex of the Newton polytope of the numerator of $C$ that corresponds to a monomial with positive coefficient (recall Remark 3.13), for instance:

$$
\left(\hat{a}^{*}, \hat{x}^{*}\right)=\left(a_{2}^{*}, a_{4}^{*}, a_{6}^{*}, a_{8}^{*}, x^{*}\right)=\left(\frac{1}{10}, 10,10, \frac{1}{10}, \frac{1}{10}, 10, \frac{1}{10}, 10,10,10, \frac{1}{10}, 10,10,10,10, \frac{1}{10}\right) .
$$

Letting $\left(a^{*}, x^{*}\right)=\phi\left(\hat{a}^{*}, \hat{x}^{*}\right)$, we have

$$
a^{*}=\left(\frac{1}{100}, \frac{1}{10}, \frac{11}{10}, 10, \frac{1001}{100}, 10, \frac{101}{10}, \frac{1}{10}, 1010,10, \frac{1}{10}, 1000,10\right),
$$

and

$$
c^{*}:=W x^{*}=\left(\frac{703}{10}, \frac{301}{10}, \frac{301}{10}\right) .
$$

Thus, by Theorem 3.12 , we obtain a witness to multistationarity via any choice of $\kappa^{*}$ for which $\bar{a}\left(\kappa^{*}\right)=a^{*}$, where $\bar{a}$ is defined in equation (5). One such $\kappa^{*}$ is as follows:

$$
\begin{gathered}
\kappa^{*}=\left(\frac{1001}{10}, \frac{1001}{100}, \frac{1001}{100}, 1,100,101000, \frac{1}{1000}, \frac{1}{10000}, \frac{1}{1000}, 11, \frac{11}{1000}, \frac{11}{1000},\right. \\
\left.\frac{1}{100}, \frac{1}{10000}, \frac{101}{100000}, 200,10,10\right) .
\end{gathered}
$$




\begin{tabular}{lll}
\hline & $C$ & $\widetilde{C}$ \\
\hline Numerator & & \\
$\quad$ total degree & 11 & 47 \\
number of terms & 704 & 246,232 \\
Denominator & & \\
total degree & 7 & 33 \\
number of terms & 1 & 1750 \\
\hline
\end{tabular}

TABle 1. Comparison for Example 3.1 of the critical functions $C$ and $\widetilde{C}$. We see that $C$ is simpler and hence easier to analyze. In particular, its denominator is a monomial and thus is easily seen to be positive on the positive orthant.

To confirm this witness, using $a^{*}$ and $c^{*}$ above, we approximately solve the polynomial system $\left.h_{c, a}\right|_{(a, c)=\left(a^{*}, c^{*}\right)}=0$ and find seven real solutions for $x$. Three of these are positive steady states:

$$
\begin{aligned}
& x^{(1)} \approx(1.18,2.25,0.46,1.54,1.56,26.55,0.016,1.31,5.81,5.81,26.52,0.0034), \\
& x^{(2)}=(0.1,10,0.1,10,10,10,0.1,10,10,10,10,0.1), \quad \text { and } \\
& x^{(3)} \approx(0.0027,14.56,0.0037,14.87,14.85,0.38,0.15,15.00,10.30,10.30,0.39,4.06) .
\end{aligned}
$$

With an eye toward comparing our approach to that of [4], we now obtain another critical function, using the procedures in [4]. Recall from Remark 3.10 that the parametrization in [4] has the form $(\kappa, \hat{x}) \mapsto\left(\kappa,(\hat{x}, \Phi(\kappa, \hat{x}))\right.$. Applying their method here, we first solve for 9 of the variables $x_{i}$ (namely, those with $i=4, \ldots, 12)$ in the 9 non-conservation-law equations $f_{c, \kappa, i}=0$, for $i=4, \ldots, 12$. This yields a steady-state parametrization:

$$
\psi: \mathbb{R}_{>0}^{21} \rightarrow \mathbb{R}_{>0}^{18} \times \mathbb{R}_{>0}^{12}
$$

of the form $\psi\left(\kappa, x_{1}, x_{2}, x_{3}\right)=(\kappa, x)$. Substituting this parametrization into $\operatorname{det} \operatorname{Jac}\left(f_{c, \kappa}\right)$ gives a critical function which we denote by $\widetilde{C}(v)$ (here we are writing $v=\left(\kappa, x_{1}, x_{2}, x_{3}\right)$ ). The denominator of $\widetilde{C}(v)$ is a polynomial in $\mathbb{Q}[v]$ that is positive for every $\kappa \in \mathbb{R}_{>0}^{18}$ and every $\left(x_{1}, x_{2}, x_{3}\right) \in \mathbb{R}_{>0}^{3}$, and has total degree 33 and 1750 terms. The numerator, also a polynomial in $\mathbb{Q}[v]$, has total degree 47 and 246232 terms.

As seen in Table 1, which compares the critical functions $C$ and $\widetilde{C}$, our critical function $C$ is simpler (fewer variables, with both numerator and denominator having lower degree and fewer terms). Moreover, the fact that the denominator of $C$ is a monomial makes it apparent that this denominator is positive on the positive orthant. In contrast, the denominator of $\widetilde{C}$ has 1750 terms (which nonetheless are all positive).

Example 3.19 (Phosphorylation of two substrates, continued). Revisiting the network in Example 2.1. we exhibit two critical functions $C$ and $\widetilde{C}$ : one that arises from a simplification $h_{c, a}$, and then the one that Conradi, Feliu, Mincheva, and Wiuf presented [4]. We will see again that $C$ is much simpler than $\widetilde{C}$. 
The first critical function. Consider the following upper-triangular matrix:

$$
M(\kappa)=\left(\begin{array}{cccccc}
\frac{1}{\kappa_{3}} & 0 & 0 & 0 & \frac{1}{\kappa_{3}} & 0 \\
0 & \frac{1}{\kappa_{9}} & 0 & 0 & 0 & \frac{1}{\kappa_{9}} \\
0 & 0 & \frac{1}{\kappa_{1}} & 0 & 0 & 0 \\
0 & 0 & 0 & \frac{1}{\kappa_{7}} & 0 & 0 \\
0 & 0 & 0 & 0 & \frac{1}{\kappa_{4}} & 0 \\
0 & 0 & 0 & 0 & 0 & \frac{1}{\kappa_{10}}
\end{array}\right) .
$$

Note that $\operatorname{det} M(\kappa)>0$ for all $\kappa \in \mathbb{R}_{>0}^{10}$. Following (8)-(10), the resulting system is

$$
\begin{array}{lll}
h_{c, a, 1}=x_{1}+x_{7}+x_{8}-c_{1}, & h_{c, a, 2}=x_{2}+x_{9}+x_{10}-c_{2}, \\
h_{c, a, 3}=x_{3}+x_{4}+x_{7}+x_{9}-c_{3}, & h_{c, a, 4}=x_{7}-a_{1} x_{9}, \\
h_{c, a, 5}=x_{5}+x_{6}+x_{8}+x_{10}-c_{4}, & h_{c, a, 6}=x_{8}-a_{2} x_{10}, \\
h_{c, a, 7}=x_{1} x_{3}-a_{3} x_{7}, & & h_{c, a, 8}=x_{1} x_{5}-a_{4} x_{8}, \\
h_{c, a, 9}=x_{2} x_{4}-a_{5} x_{9}, & h_{c, a, 10}=x_{2} x_{6}-a_{6} x_{10},
\end{array}
$$

where the effective parameters are:

$$
\bar{a}_{1}=\frac{\kappa_{6}}{\kappa_{3}}, \quad \bar{a}_{2}=\frac{\kappa_{12}}{\kappa_{9}}, \quad \bar{a}_{3}=\frac{\kappa_{2}+\kappa_{3}}{\kappa_{1}}, \quad \bar{a}_{4}=\frac{\kappa_{8}+\kappa_{9}}{\kappa_{7}}, \quad \bar{a}_{5}=\frac{\kappa_{5}+\kappa_{6}}{\kappa_{4}}, \quad \bar{a}_{6}=\frac{\kappa_{11}+\kappa_{12}}{\kappa_{10}} .
$$

Notice that the map $\mathbb{R}_{>0}^{12} \rightarrow \mathbb{R}_{>0}^{6}$ given by $\kappa \mapsto \bar{a}(\kappa)$ is surjective. The Jacobian matrix of $h_{c, a}$ is

$$
\operatorname{Jac}\left(h_{c, a}\right)=\left(\begin{array}{cccccccccc}
1 & 0 & 0 & 0 & 0 & 0 & 1 & 1 & 0 & 0 \\
0 & 1 & 0 & 0 & 0 & 0 & 0 & 0 & 1 & 1 \\
0 & 0 & 1 & 1 & 0 & 0 & 1 & 0 & 1 & 0 \\
0 & 0 & 0 & 0 & 0 & 0 & 1 & 0 & -a_{1} & 0 \\
0 & 0 & 0 & 0 & 1 & 1 & 0 & 1 & 0 & 1 \\
0 & 0 & 0 & 0 & 0 & 0 & 0 & 1 & 0 & -a_{2} \\
x_{3} & 0 & x_{1} & 0 & 0 & 0 & -a_{3} & 0 & 0 & 0 \\
x_{5} & 0 & 0 & 0 & x_{1} & 0 & 0 & -a_{4} & 0 & 0 \\
0 & x_{4} & 0 & x_{2} & 0 & 0 & 0 & 0 & -a_{5} & 0 \\
0 & x_{6} & 0 & 0 & 0 & x_{2} & 0 & 0 & 0 & -a_{6}
\end{array}\right) .
$$

By solving the equations $h_{c, a, 4}=h_{c, a, 6}=h_{c, a, 7}=h_{c, a, 8}=h_{c, a, 9}=h_{c, a, 10}=0$ in the unknowns $a_{1}, a_{2}, a_{3}, a_{4}, a_{5}, a_{6}$, we obtain

$$
a_{1}=\frac{x_{7}}{x_{9}}, \quad a_{2}=\frac{x_{8}}{x_{10}}, \quad a_{3}=\frac{x_{1} x_{3}}{x_{7}}, \quad a_{4}=\frac{x_{1} x_{5}}{x_{8}}, \quad a_{5}=\frac{x_{2} x_{4}}{x_{9}}, \quad a_{6}=\frac{x_{2} x_{6}}{x_{10}} .
$$

This yields a steady-state parametrization $\phi: \mathbb{R}_{>0}^{10} \rightarrow \mathbb{R}_{>0}^{6} \times \mathbb{R}_{>0}^{10}$, where $\hat{a}=\emptyset$ and $\hat{x}=x$, so we denote $\phi(\hat{a}, \hat{x})$ by $\phi(x)=\left(a_{1}, \ldots, a_{6}, x\right)$, which is defined as follows:

$$
\left(a_{1}=\frac{x_{7}}{x_{9}}, a_{2}=\frac{x_{8}}{x_{10}}, a_{3}=\frac{x_{1} x_{3}}{x_{7}}, a_{4}=\frac{x_{1} x_{5}}{x_{8}}, a_{5}=\frac{x_{2} x_{4}}{x_{9}}, a_{6}=\frac{x_{2} x_{6}}{x_{10}} ; x\right) .
$$

We substitute the parametrization $\phi(x)$ into $\operatorname{det} \operatorname{Jac}\left(h_{c, a}\right)$ to obtain $C(x)=-\frac{x_{1} x_{2}}{x_{9} x_{10}} B(x)$, where $B(x)$ is the following polynomial:

$$
\begin{gathered}
x_{1} x_{10} x_{2} x_{3}+x_{1} x_{10} x_{2} x_{4}+x_{1} x_{10} x_{2} x_{7}+x_{1} x_{10} x_{2} x_{9}+x_{1} x_{10} x_{3} x_{6}+x_{1} x_{10} x_{4} x_{6}+x_{1} x_{10} x_{4} x_{9}+ \\
x_{1} x_{10} x_{6} x_{7}+x_{1} x_{10} x_{6} x_{9}+x_{1} x_{2} x_{3} x_{5}+x_{1} x_{2} x_{3} x_{6}+x_{1} x_{2} x_{3} x_{8}+x_{1} x_{2} x_{4} x_{5}+x_{1} x_{2} x_{4} x_{6}+x_{1} x_{2} x_{4} x_{8}+ \\
x_{1} x_{2} x_{5} x_{7}+x_{1} x_{2} x_{5} x_{9}+x_{1} x_{2} x_{6} x_{7}+x_{1} x_{2} x_{6} x_{9}+x_{1} x_{2} x_{7} x_{8}+x_{1} x_{2} x_{8} x_{9}+x_{1} x_{4} x_{5} x_{9}+x_{1} x_{4} x_{6} x_{9}+ \\
x_{1} x_{4} x_{8} x_{9}+x_{10} x_{2} x_{3} x_{7}+x_{10} x_{3} x_{6} x_{7}-x_{10} x_{4} x_{5} x_{7}+x_{2} x_{3} x_{5} x_{7}+x_{2} x_{3} x_{5} x_{8}+x_{2} x_{3} x_{6} x_{7}+x_{2} x_{3} x_{7} x_{8}+ \\
x_{2} x_{4} x_{5} x_{8}+x_{2} x_{5} x_{7} x_{8}+x_{2} x_{5} x_{8} x_{9}-x_{3} x_{6} x_{8} x_{9}+x_{4} x_{5} x_{8} x_{9} .
\end{gathered}
$$

We see that $B(x)$ is square-free and homogeneous, so every monomial of $B(x)$ defines a vertex of $\mathrm{NP}(B(x))$. So, as $B(x)$ has both positive and negative terms (underlined), we conclude from Lemma 2.2 that $B(x)$, and thus $C(x)$ too, changes sign. So, by Theorem 3.12 (this network is 
conservative and hence dissipative, and has no boundary steady states [4, 35]), the network is multistationary. We will see that such properties of $B(x)$ - having square-free monomials and homogeneous - come from the fact that every non-conservation-law $h_{c, a, i}$ is a binomial (Theorem 4.6).

The second critical function (from [4]). Using the equations for $f_{c, \kappa}$ given in Example 2.1. the resulting Jacobian matrix is as follows:

$$
\operatorname{Jac}\left(f_{c, \kappa}\right)=\left(\begin{array}{cccccccccc}
1 & 0 & 0 & 0 & 0 & 0 & 1 & 1 & 0 & 0 \\
0 & 1 & 0 & 0 & 0 & 0 & 0 & 0 & 1 & 1 \\
0 & 0 & 1 & 1 & 0 & 0 & 1 & 0 & 1 & 0 \\
0 & -\kappa_{4} x_{4} & 0 & -\kappa_{4} x_{2} & 0 & 0 & \kappa_{3} & 0 & \kappa_{5} & 0 \\
0 & 0 & 0 & 0 & 1 & 1 & 0 & 1 & 0 & 1 \\
0 & -\kappa_{10} x_{6} & 0 & 0 & 0 & -\kappa_{10} x_{2} & 0 & \kappa_{9} & 0 & \kappa_{11} \\
\kappa_{1} x_{3} & 0 & \kappa_{1} x_{1} & 0 & 0 & 0 & -\kappa_{2}-\kappa_{3} & 0 & 0 & 0 \\
\kappa_{7} x_{5} & 0 & 0 & 0 & \kappa_{7} x_{1} & 0 & 0 & -\kappa_{8}-\kappa_{9} & 0 & 0 \\
0 & \kappa_{4} x_{4} & 0 & \kappa_{4} x_{2} & 0 & 0 & 0 & 0 & -\kappa_{5}-\kappa_{6} & 0 \\
0 & \kappa_{10} x_{6} & 0 & 0 & 0 & \kappa_{10} x_{2} & 0 & 0 & 0 & -\kappa_{11}-\kappa_{12}
\end{array}\right) .
$$

In [4], a steady state parametrization of the form $(\kappa, \hat{x}) \mapsto\left(\kappa,(\hat{x}, \Phi(\kappa, \hat{x}))\right.$, where $\hat{x}=\left(x_{1}, x_{2}, x_{3}, x_{5}\right)$, is derived as follows. The equations $f_{c, \kappa, i}=0(i=4,6,7,8,9,10)$ are solved for the unknowns $x_{i}$ $(i=4,6,7,8,9,10)$, which yields a steady-state parametrization,

$$
\psi: \mathbb{R}_{>0}^{16} \longrightarrow \mathbb{R}_{>0}^{12} \times \mathbb{R}_{>0}^{10}
$$

where $\psi\left(\kappa ; x_{1}, x_{2}, x_{3}, x_{5}\right)$ is defined as

$$
\left(\kappa ; x_{1}, x_{2}, x_{3}, \frac{\kappa_{1} \kappa_{3}\left(\kappa_{5}+\kappa_{6}\right) x_{1} x_{3}}{\kappa_{4} \kappa_{6}\left(\kappa_{2}+\kappa_{3}\right) x_{2}}, x_{5}, \frac{\left(\kappa_{11}+\kappa_{12}\right) \kappa_{7} \kappa_{9} x_{1} x_{5}}{\left(\kappa_{8}+\kappa_{9}\right) \kappa_{10} \kappa_{12} x_{2}}, \frac{\kappa_{1} x_{1} x_{3}}{\kappa_{2}+\kappa_{3}}, \frac{\kappa_{7} x_{1} x_{5}}{\kappa_{8}+\kappa_{9}}, \frac{\kappa_{1} \kappa_{3} x_{1} x_{3}}{\left(\kappa_{2}+\kappa_{3}\right) \kappa_{6}}, \frac{\kappa_{1} \kappa_{7} \kappa_{9} x_{1} x_{5}}{\left(\kappa_{8}+\kappa_{9}\right) \kappa_{12}}\right) .
$$

Substituting $(\kappa, x)=\psi\left(\kappa, x_{1}, x_{2}, x_{3}, x_{5}\right)$ into $\operatorname{det} \operatorname{Jac}\left(f_{c, \kappa}\right)$ yields the critical function $\widetilde{C}\left(\kappa ; x_{1}, x_{2}, x_{3}, x_{5}\right)$, which is exactly the function " $a(\hat{x})$ " in [4, $\S 6$ in Supplementary Information]. This critical function $\widetilde{C}$ is a rational function, and the denominator is $\left(\kappa_{2}+\kappa_{3}\right) \kappa_{6}\left(\kappa_{8}+\kappa_{9}\right) \kappa_{12} x_{2}$ and thus is positive for every choice of positive $\left(\kappa ; x_{1}, x_{2}, x_{3}, x_{5}\right) \in \mathbb{R}_{>0}^{16}$. The numerator is a polynomial in $\mathbb{Q}\left[\kappa ; x_{1}, x_{2}, x_{3}, x_{5}\right]$ with total degree 15 and 225 terms. Conradi, Feliu, Mincheva, and Wiuf showed that $\widetilde{C}$ changes sign, and so again by Theorem 3.12 (or [4, Corollary 2]) the network is multistationary.

We compare the two critical functions $C$ and $\widetilde{C}$ described above:

(1) $C$ is simpler than $\widetilde{C}$. The numerator of $C$ has 36 terms while that of $\widetilde{C}$ has 225 terms.

(2) Unlike the numerator of $\widetilde{C}$, the numerator of $C$ (namely, $B(x)$ ) is square-free and homogeneous. So, to conclude that the network is multistationary, we only had to examine the signs in $B(x)$, whereas in [4] a more careful examination of the Newton polytope of the numerator of $\widetilde{C}$ had to be undertaken.

(3) The critical function $\widetilde{C}$ contains the rate-constant vector $\kappa$, so the authors of [4] used the structure of $\widetilde{C}$ to derive necessary and sufficient conditions on $\kappa$ for multistationarity - in other words, they found the multistationary region of parameter space. In contrast, $C$ does not contain $\kappa$, so we can not readily use $C$ to find the multistationary region. Nonetheless, we can use $C$ to find a witness to multistationarity (by Theorem 3.12).

3.3. Critical functions and discriminants. For readers familiar with real algebraic geometry, we now explain the relationship between a critical function $C(\hat{a}, \hat{x})$ and the mixed discriminant of the polynomial equation system $h_{c, a}(x)=0$ [18]. Let $I$ be the ideal in the polynomial ring 
$\mathbb{Q}\left[c, a, x_{1}, x_{1}^{-1}, \ldots, x_{s}, x_{s}^{-1}, y_{1}, y_{1}^{-1}, \ldots, y_{s}, y_{s}^{-1}\right]$ generated by $h_{c, a, 1}(x), \ldots, h_{c, a, s}(x)$ and the equations expressing that $y=\left(y_{1}, \ldots, y_{s}\right)$ lies in the kernel of the Jacobian matrix $\operatorname{Jac}\left(h_{c, a}\right)$ with respect to the $x$ variables. Note that the vanishing of these equations for given values $\left(c^{*}, a^{*}\right)$ of $(c, a)$ at $x^{*}, y^{*}$ imply that $\operatorname{det}\left(\operatorname{Jac}\left(h_{c^{*}, a^{*}}\right)\right)\left(x^{*}\right)=0$ and so $x^{*}$ is a non-simple common root of $h_{c^{*}, a^{*}}$. If the elimination ideal $I \cap \mathbb{Q}[c, a]$ has codimension one, we call any generator of this ideal a mixed discriminant of the family $h_{c, a}(x)$, and we denote it by $D(c, a)$. So, $D\left(c^{*}, a^{*}\right)=0$ whenever $h_{c^{*}, a^{*}}$ has a multiple root over $\mathbb{C}^{*}$ where all maximal minors of size $(s-1)$ of the Jacobian matrix are nonzero. As a real positive root $x^{*}$ of $h_{c^{*}, a^{*}}(x)$ is a real positive root of $f_{k^{*}}(x)=0$ when $\bar{a}\left(k^{*}\right)=a^{*}$, and the matrix $M\left(\kappa^{*}\right)$ is invertible, such a positive multiple root $x^{*}$ of $h_{c^{*}, a^{*}}$ is a degenerate steady state of the dynamical system $\dot{x}=f_{\kappa^{*}}(x)$, and in this case $D\left(c^{*}, a^{*}\right)=0$.

Assume that the codimension of the elimination ideal is one and consider the discriminant locus, i.e., the set of pairs of vectors of parameters $\left(c^{*}, a^{*}\right)$ where the discriminant vanishes $\left(D\left(c^{*}, a^{*}\right)=0\right)$. Consider also the critical locus, i.e., the set of pairs $(\hat{a}, \hat{x})$ where the critical function vanishes $(C(\hat{a}, \hat{x})=0)$. We relate these two conditions in Proposition 3.21 below. We begin with a simple example.

Example 3.20. Consider the following network [26, where we set two of the rate constants to 1 to simplify the analyses:

$$
0 \stackrel{1}{\underset{\kappa}{\rightleftharpoons}} \mathrm{A}, \quad 2 \mathrm{~A} \stackrel{1}{\longrightarrow} 3 \mathrm{~A} .
$$

The resulting mass-action kinetics ODE is:

$$
\frac{d x_{A}}{d t}=x_{A}^{2}-\kappa x_{A}+1,
$$

and the discriminant equals $\kappa^{2}-4$. We obtain, from the ODE (16), the steady-state parametrization $\phi: \mathbb{R}_{>0} \rightarrow \mathbb{R}_{>0}^{2}$ given by:

$$
x_{A} \mapsto\left(x_{A}, \kappa=\frac{x_{A}^{2}+1}{x_{A}}\right) .
$$

(Here, $\bar{a}$ is the identity map $\bar{a}(\kappa)=\kappa$, and $\hat{a}=\emptyset, \hat{x}=x_{A}$.) This steady-state parametrization is depicted in Figure 1, along with the unique degenerate steady state (when $\left(x_{A}, \kappa\right)=(1,2)$ ) and the corresponding projections onto the critical locus $\left(x_{A}=1\right)$ and onto the discriminant locus $(\kappa=2)$. The second point on the discriminant locus, $\kappa=-2$, is not shown.

We see in Figure 1 that the critical locus is a projection of the degenerate steady states, and the discriminant locus is a subset of a similar projection. The first observation - viewing the critical locus as a projection of the degenerate steady states - gives an intrinsic definition of the critical locus, which does not depend on the specific parametrization. As for the second observation, pertaining to the discriminant locus, we generalize this in Proposition 3.21 .

Proposition $3.21(C=0$ implies $D=0)$. Consider a network $G$ with conservation-law matrix $W$ and simplified system $h_{c, a}(x)$ as in Definition 3.2. Assume $I \cap \mathbb{Q}[c, a]$ has codimension one, and let $D(c, a)$ denote the mixed discriminant. Consider, as in Definition 3.6, a positive parametrization $\phi: \mathbb{R}_{>0}^{\hat{m}} \times \mathbb{R}_{>0}^{\hat{s}} \rightarrow \mathbb{R}_{>0}^{\bar{m}} \times \mathbb{R}_{>0}^{s}$, denoted by $(\hat{a}, \hat{x}) \mapsto \phi(\hat{a}, \hat{x})$, with respect to $h_{c, a}$. Let $C(\hat{a}, \hat{x})$ denote the resulting critical function. Then, for $\left(\hat{a}^{*}, \hat{x}^{*}\right) \in \mathbb{R}_{>0}^{\hat{m}} \times \mathbb{R}_{>0}^{\hat{s}}$, if $C\left(\hat{a}^{*}, \hat{x}^{*}\right)=0$ and any 


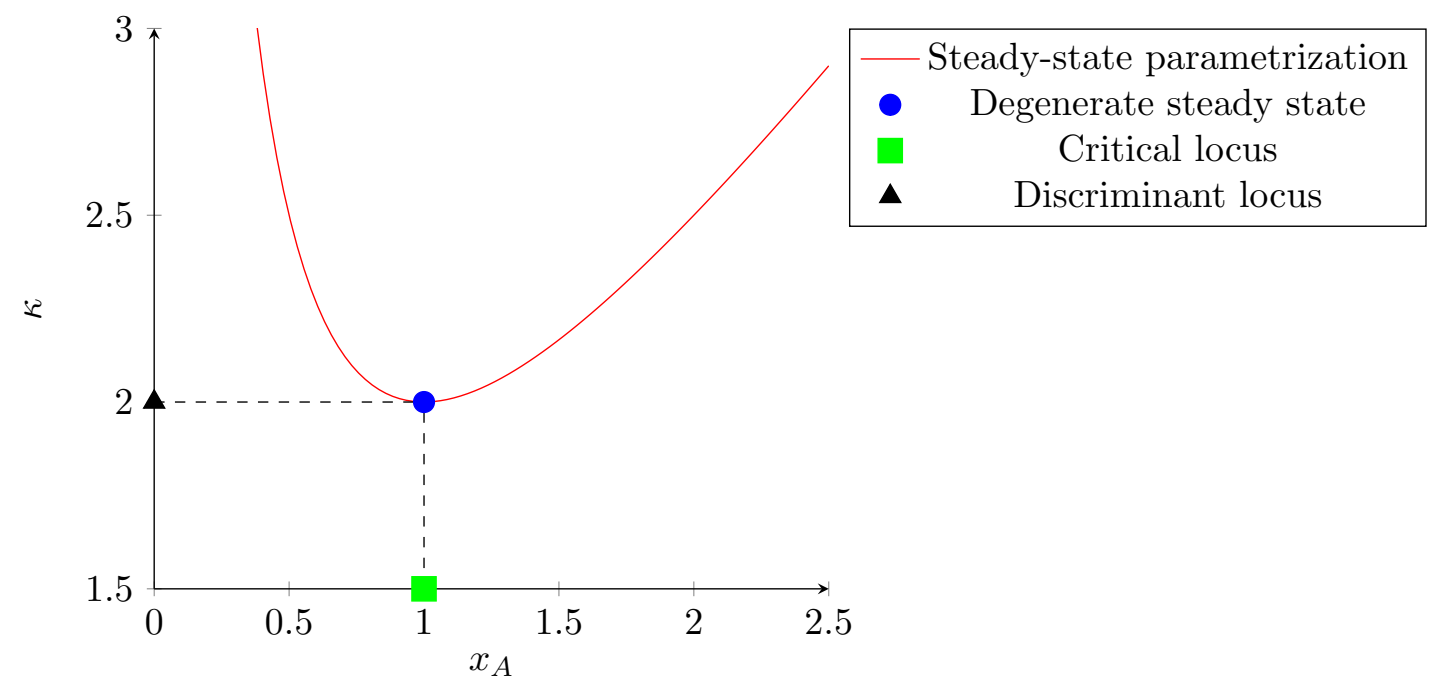

Figure 1. The image of the steady-state parametrization (17) for network (15). Also shown is the unique degenerate steady state and its projections to the critical locus (where $C=0$ ) and discriminant locus (where $D=0$ ).

minor of the Jacobian matrix $\operatorname{Jac}\left(h_{\hat{c}^{*}, \hat{a}^{*}}\right)\left(\hat{x}^{*}\right)$ of size $(s-1)$ is nonzero, then $D\left(c^{*}, a^{*}\right)=0$, where $\left(a^{*}, x^{*}\right)=\phi\left(\hat{a}^{*}, \hat{x}^{*}\right)$ and $c^{*}=W x^{*}$.

Proof. If $C\left(\hat{a}^{*}, \hat{x}^{*}\right)=0$, then, by definition, $\left.\left(\operatorname{det} \operatorname{Jac}\left(h_{c, a}\right)\right)\right|_{(a, x)=\phi\left(\hat{a}^{*}, \hat{x}^{*}\right)}=0$. Let $\left(a^{*}, x^{*}\right)=$ $\phi\left(\hat{a}^{*}, \hat{x}^{*}\right)$. Then, for $c^{*}=W x^{*}$, the system $h_{c^{*}, a^{*}}=0$ has a multiple root (namely, $\left.x^{*}\right)$. So, by the definition of the mixed discriminant and the assumptions about the Jacobian matrix $\operatorname{Jac}\left(h_{\hat{c}^{*}, \hat{a}^{*}}\right)\left(\hat{x}^{*}\right)$, we have $D\left(c^{*}, a^{*}\right)=0$.

The converse of Proposition 3.21 does not hold, as we see in the following example.

Example 3.22 (Converse of Proposition 3.21 is false). We revisit the network in Examples 2.1 and 3.19. For the following choice of $\kappa^{*}$ and $c^{*}$ :

$$
\begin{aligned}
\kappa^{*} & =\left(1, \frac{1}{20}, \frac{1}{20}, 1, \frac{19}{2}, \frac{1}{2}, 1,5,5,6, \frac{926}{823}, \frac{4630}{823}\right) \quad \text { and } \\
c^{*} & =\left(12, \frac{2675}{926}, 22, \frac{11935}{926}\right),
\end{aligned}
$$

there are two positive steady states:

$$
\begin{aligned}
& x^{(1)}=\left(1,1,1,10,10,1,10,1,1, \frac{823}{926}\right) \text { and } \\
& x^{(2)} \approx(2.48,0.60,0.31,13.08,6.84,2.81,7.82,1.70,0.78,1.51) .
\end{aligned}
$$

The first steady state, $x^{(1)}$, is degenerate, while the second, $x^{(2)}$, is nondegenerate. Thus, for the parametrization (14), which we denote by $x \mapsto \phi(x)$, it follows that $C\left(x^{(1)}\right)=0$ (because $x^{(1)}$ is 
degenerate). Let $\left(a^{(1)}, x^{(1)}\right)=\phi\left(x^{(1)}\right)$ and $\left(a^{(2)}, x^{(2)}\right)=\phi\left(x^{(2)}\right)$. So,

$$
D\left(W x^{(2)}, a^{(2)}\right)=D\left(W x^{(1)}, a^{(1)}\right)=D\left(c^{*}, a^{(1)}\right)=0,
$$

where we are using the fact that $a^{(1)}=a^{(2)}$ and $W x^{(1)}=W x^{(2)}=c^{*}$. However, the second steady state, $x^{(2)}$, is nondegenerate, so although $D\left(W x^{(2)}, a^{(2)}\right)=0$, we nonetheless have $C\left(x^{(2)}\right) \neq 0$.

\section{Multistationarity FOR Linearly Binomial Networks}

This section focuses on a class of networks that appear surprisingly often in applications. We called these networks linearly binomial networks in Definition 3.4 because their steady-state equations are equivalent to binomial equations, and these binomial equations are related to the polynomials arising from mass-action kinetics linearly via multiplication by a matrix $M(\kappa)$. We prove for linearly binomial networks that multistationarity can be decided simply from inspecting the critical function (Theorem 4.6) and that the regions of parameter space allowing for multistationarity that we find via degree theory and the use of effective parameters are full-dimensional (Theorem 4.10).

It follows from Definition 3.4 that linearly binomial networks are the networks for which the augmented system $f_{c, \kappa}$ can be simplified to some $h_{c, a}$, as in 10 , with the following form:

$$
h_{c, a}(x)_{i}= \begin{cases}x^{\gamma_{k}}-a_{k} \cdot x^{\delta_{k}} & \text { if } \left.i=j_{k} \in\left\{j_{1}, j_{2}, \ldots, j_{s-d}\right\} \quad \text { (i.e., } i \notin I\right) \\ (W x-c)_{k} & \text { if } i=i_{k} \in I .\end{cases}
$$

where for $k=1,2, \ldots, s-d$, the coefficient $a_{k}$ is an indeterminate arising from an effective parameter $\bar{a}_{i}(\kappa)$, and both $x^{\gamma_{k}}$ and $x^{\delta_{k}}$ are monomials. The steady-state equations can be transformed into binomial equations by linear operations, as in (8), via a matrix $M(\kappa)$ which has positive determinant for all $\kappa \in \mathbb{R}_{>0}^{m}$.

Remark 4.1. Mass-action systems arising from linearly binomial networks have "toric steady states", as defined in [36], for every choice of positive rate constants. For networks with toric steady states, analyzing and finding witnesses for multistationarity has been investigated [32, 35, 36].

We use the binomials in (18) to solve for the indeterminates $a_{k}$ :

$$
a_{k}=x^{\gamma_{k}-\delta_{k}}, \quad k=1,2, \ldots, s-d,
$$

and this yields a steady-state parametrization $\phi(\hat{a}, \hat{x})$, where $\hat{a}=\emptyset$ and $\hat{x}=x$ :

$$
\begin{aligned}
\phi: \mathbb{R}_{>0}^{s} & \rightarrow \mathbb{R}_{>0}^{s-d} \times \mathbb{R}_{>0}^{s} \\
(\hat{a}, \hat{x}) & \mapsto\left(\phi_{a}(x), x\right)
\end{aligned}
$$

given by $\phi_{a}(x):=\left(x^{\gamma_{1}-\delta_{1}}, x^{\gamma_{2}-\delta_{2}}, \ldots, x^{\gamma_{s-d}-\delta_{s-d}}\right)$. The parametrization $\phi(\hat{a}, \hat{x})$, which we denote by $\phi(x)$, yields the following critical function:

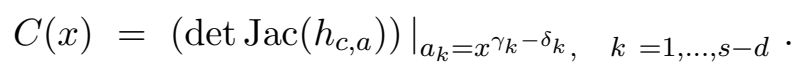

Example 4.2 (Phosphorylation of two substrates, continued). The network describing phosphorylation/dephosphorylation of two substrates, from Examples 2.1 and 3.19, is linearly binomial. This can be seen from the system $h_{c, a}$ given in Example 3.19. Moreover, the steady-state parametrization given there in (14), has the form in 20).

Example 4.3 (ERK network, continued). The ERK network from Example 3.1 is MESSI (see Section 5.1 but not linearly binomial. This result can be checked using results from [35]. 
4.1. Establishing multistationarity using the critical function. For linearly binomial networks, we show that the critical function $C(x)$ has the same sign as a homogeneous polynomial $B(x)$ with square-free monomials (Lemma 4.4). It follows that every monomial of $B(x)$ defines a vertex of the Newton polytope, so establishing multistationarity for a linearly binomial network is easy: simply check whether $B(x)$ has coefficients of both signs (Theorem 4.6).

Recall that a polynomial $g(x)$ is homogeneous of degree $d$ if $g(\lambda x)=\lambda^{d} g(x)$ for all constants $\lambda \in \mathbb{C}$.

Lemma 4.4. For a linearly binomial network $G$, the critical function $C(x)$ in (20) has the form

$$
C(x)=\frac{x^{\alpha}}{x^{\beta}} \cdot B(x),
$$

where $x^{\alpha}$ and $x^{\beta}$ are monomials, and $B(x)$ is either the zero polynomial or a homogeneous polynomial of degree $d=s-\operatorname{rank}(N)$ such that all monomials have exponents vectors with coordinates 0,1 . Here, $s$ denotes the number of species, and $N$ denotes the stoichiometric matrix of $G$.

Proof. First we reorder the polynomials $h_{c, a}$ as $b_{1}, b_{2}, \ldots, b_{s}$ such that the first $s-d$ polynomials are the binomials:

$$
b_{k}=x^{\gamma_{k}}-a_{k} \cdot x^{\delta_{k}}, \quad k=1,2, \ldots, s-d,
$$

and the remaining $d$ polynomials are the linear equations from the conservation laws:

$$
b_{s-d+\ell}=(W x-c)_{\ell}, \quad \ell=1,2, \ldots, d .
$$

As $b$ is obtained by reordering the polynomials $h_{c, a}$, we have that

$$
\operatorname{det} \operatorname{Jac}\left(h_{c, a}\right)=(-1)^{\mu} \cdot \operatorname{det} \operatorname{Jac}(b),
$$

for some integer $\mu$. So, the critical function, as in (20), is:

$$
C(x)=\left.\left(\operatorname{det} \operatorname{Jac}\left(h_{c, a}\right)\right)\right|_{a_{k}=x^{\gamma_{k}-\delta_{k}}}=\left.(-1)^{\mu} \cdot(\operatorname{det} \operatorname{Jac}(b))\right|_{a_{k}=x^{\gamma_{k}}-\delta_{k}} .
$$

We first describe the first $s-d$ rows of $\operatorname{Jac}(b)$. For every $k=1,2, \ldots, s-d$ and for every $r=$ $1,2, \ldots, s$, the entry in the $k$-th row and the $r$-th column of $\operatorname{Jac}(b)$ is

$$
\frac{\partial b_{k}}{\partial x_{r}}=\gamma_{k, r} \frac{x^{\gamma_{k}}}{x_{r}}-a_{k} \delta_{k, r} \frac{x^{\delta_{k}}}{x_{r}} .
$$

Thus, the entry in the $k$-th row and the $r$-th column of $\left.\operatorname{Jac}(b)\right|_{a_{k}=x^{\gamma_{k}-\delta_{k}}}$ is

$$
\left.\frac{\partial b_{k}}{\partial x_{r}}\right|_{a_{k}=x^{\gamma_{k}-\delta_{k}}}=\frac{x^{\gamma_{k}}}{x_{r}}\left(\gamma_{k, r}-\delta_{k, r}\right) .
$$

As for the last $d$ rows of $\operatorname{Jac}(b)$, this submatrix is exactly $W$, the conservation-law matrix. So,

$$
\left.\operatorname{Jac}(b)\right|_{a_{k}=x^{\gamma_{k}-\delta_{k}}}=\left(\begin{array}{ccc}
\frac{x^{\gamma_{1}}}{x_{1}}\left(\gamma_{1,1}-\delta_{1,1}\right) & \ldots & \frac{x^{\gamma_{1}}}{x_{s}}\left(\gamma_{1, s}-\delta_{1, s}\right) \\
\vdots & \ddots & \vdots \\
\frac{x^{\gamma_{s-d}}}{x_{1}}\left(\gamma_{s-d, 1}-\delta_{s-d, 1}\right) & \ldots & \frac{x^{\gamma_{s-d}}}{x_{s}}\left(\gamma_{s-d, s}-\delta_{s-d, s}\right) \\
& W &
\end{array}\right),
$$


and hence,

$$
\begin{aligned}
& \left.(\operatorname{det} \operatorname{Jac}(b))\right|_{a_{k}=x^{\gamma_{k}-\delta_{k}}}=\prod_{k=1}^{s-d} x^{\gamma_{k}} \operatorname{det}\left(\begin{array}{ccc}
\frac{1}{x_{1}}\left(\gamma_{1,1}-\delta_{1,1}\right) & \ldots & \frac{1}{x_{s}}\left(\gamma_{1, s}-\delta_{1, s}\right) \\
\vdots & \ddots & \vdots \\
\frac{1}{x_{1}}\left(\gamma_{s-d, 1}-\delta_{s-d, 1}\right) & \ldots & \frac{1}{x_{s}}\left(\gamma_{s-d, s}-\delta_{s-d, s}\right) \\
\hline & W &
\end{array}\right) \\
& =\frac{\prod_{k=1}^{s-d} x^{\gamma_{k}}}{\prod_{r=1}^{s} x_{r}} \operatorname{det}\left(\begin{array}{ccc}
\left(\gamma_{1,1}-\delta_{1,1}\right) & \cdots & \left(\gamma_{1, s}-\delta_{1, s}\right) \\
\vdots & \ddots & \vdots \\
\left(\gamma_{s-d, 1}-\delta_{s-d, 1}\right) & \cdots & \left(\gamma_{s-d, s}-\delta_{s-d, s}\right) \\
& W x &
\end{array}\right)
\end{aligned}
$$

where $W x$ denotes the $d \times s$ matrix obtained from $W$ by multiplying the $j$-th column by $x_{j}$, for every $j=1,2, \ldots, s$.

Therefore, in the determinant in (22), each term is a product of $d$ distinct $x_{i}$ 's times a number, that is, we get a homogeneous polynomial $\widetilde{B}(x)$ of degree $d$ where each monomial has exponents vectors with coordinates 0,1 , as stated. Hence,

$$
C(x)=\left.(-1)^{\mu} \cdot(\operatorname{det} \operatorname{Jac}(b))\right|_{a_{k}=x^{\gamma_{k}-\delta_{k}}}=(-1)^{\mu} \cdot \frac{\Pi_{k=1}^{s-d} x^{\gamma_{k}}}{\prod_{r=1}^{s} x_{r}} \widetilde{B}(x) .
$$

Then, letting $B(x)=(-1)^{\mu} \widetilde{B}(x)$, it follows that $B(x)$ is as in 21 and is homogeneous of degree $d$ with each monomial has exponents vectors with coordinates 0,1 .

Notice that $C(x)$ and $B(x)$, as in $(21)$, have the same sign for every $x \in \mathbb{R}_{>0}^{s}$. This observation motivates the following definition.

Definition 4.5. The critical polynomial of a linearly binomial network is the polynomial $B(x)$ in (21).

In the remainder of this section, we make the following assumption: $B(x)$ is not the zero polynomial. Indeed, we do not know of any network for which the critical function is the zero function. For such a network, every steady state (in every compatibility class and for every choice of rate constants) would be degenerate. We suspect that no such networks exist, and in fact the non-existence of such networks is implied by the (open) Nondegeneracy Conjecture [28].

Theorem 4.6 (Multistationarity for linearly binomial networks). Let $G$ be a linearly binomial network that is dissipative and has no boundary steady states in any compatibility class. Let $B(x)$ denote its critical polynomial. Then:

(A) Multistationarity. $G$ is multistationary if and only if $B(x)$ has a coefficient with sign equal to $(-1)^{\operatorname{rank}(N)+1}$, where $N$ denotes the stoichiometric matrix of $G$.

(B) Witness to multistationarity. Every $x^{*} \in \mathbb{R}_{>0}^{s}$ with $\operatorname{sign}\left(B\left(x^{*}\right)\right)=(-1)^{\operatorname{rank}(N)+1}$ yields a witness to multistationarity $\left(\kappa^{*}, c^{*}\right)$ as follows: let $c^{*}=W x^{*}$ (where $W$ is the conservationlaw matrix), and let $\kappa^{*} \in \mathbb{R}_{>0}^{m}$ be such that $\bar{a}\left(\kappa^{*}\right)=\phi_{a}\left(x^{*}\right)$ (where $\phi_{a}$ is as in (19)). 
Proof. Part (B) follows directly from Theorem 3.12 and Lemma 4.4 .

For the forward direction of part (A), we proceed by contrapositive. Assume that every coefficient of $B(x)$ has sign $(-1)^{\operatorname{rank}(N)}$ (at least one such coefficient exists, as $B(x)$ is nonzero). So, $B(x)$, and thus $C(x)$ as well (by Lemma 4.4), has $\operatorname{sign}(-1)^{\operatorname{rank}(N)}$ for all $x \in \mathbb{R}_{>0}^{s}$. Thus, by Theorem 3.12 . $G$ is monostationary.

For the backward direction of part (A), assume that $B(x)$ has a coefficient with sign equal to $(-1)^{\operatorname{rank}(N)+1}$. By Lemma $4.4, B(x)$ is homogeneous with square-free monomials, so every monomial of $B(x)$ defines a vertex of the Newton polytope. Then, by Lemma 2.2 , there exists $x^{*} \in \mathbb{R}_{>0}^{s}$ with $\operatorname{sign}\left(B\left(x^{*}\right)\right)=(-1)^{\operatorname{rank}(N)+1}$. By again appealing to Lemma 4.4, we conclude that $C(x)$ also takes that sign, so, by Theorem 3.12 , $G$ is multistationary.

Theorem 4.6 and its proof yield the following procedure for obtaining, for linearly binomial networks, a witness to multistationarity.

Procedure 4.7 (Witness to multistationarity for linearly binomial networks).

Input: A binomial network that is dissipative and has no boundary steady states, given by equations $h_{c, a}(x)$ as in (18), arising from a reparametrization map $\bar{a}$ as in (7), with conservation-law matrix $W$ and steady-state parametrization $\phi(x)=\left(\phi_{a}(x), x\right)$ as in (19).

Output: "No" if $G$ is not multistationary; otherwise, a witness to multistationarity.

Steps:

(1) Does the critical polynomial $B(x)$ (Definition 3.8) have a coefficient with sign equal to $(-1)^{\operatorname{rank}(N)+1}$ (e.g., if $B(x)$ has both positive and negative coefficients)? If not, return "No". If yes, pick any $x^{*} \in \mathbb{R}_{>0}^{s} \operatorname{such}$ that $\operatorname{sign}\left(B\left(x^{*}\right)\right)=(-1)^{\operatorname{rank}(N)+1}$.

(2) Let $c^{*}:=W x^{*}$.

(3) Pick any $\kappa^{*} \in \mathbb{R}_{>0}^{m}$ such that $\bar{a}\left(\kappa^{*}\right)=\phi_{a}\left(x^{*}\right)$.

(4) Return $\left(\kappa^{*}, c^{*}\right)$.

Recall from Remark 3.13 that one way to complete Step (1) (in the "yes" case) follows the proof of Lemma 2.2. Namely, choose a monomial $x^{\alpha}$ of $B(x)$ with coefficient having sign $(-1)^{\mathrm{rank}(N)+1}$, and then pick $x^{*} \in \mathbb{R}_{>0}^{s}$ as follows: let $\lambda \gg 1$ and $x_{i}^{*}=\lambda$ when $i \in \alpha$ and $x_{i}^{*}=1$ when $i \notin \alpha$. It follows that $\left(x^{*}\right)^{\alpha} \gg\left(x^{*}\right)^{\beta}$ for every other monomial $x^{\beta}$ in $B(x)$, and hence $\operatorname{sign}\left(B\left(x^{*}\right)\right)=(-1)^{\operatorname{rank}(N)+1}$.

We demonstrate Procedure 4.7 in the following example.

Example 4.8. Consider the following network from [14, Figure 1(k)]:

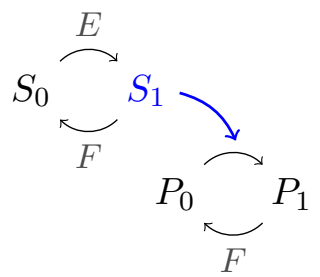

$$
\begin{aligned}
\mathrm{S}_{0}+\mathrm{E} \underset{\kappa_{2}}{\stackrel{\kappa_{1}}{\rightleftharpoons}} \mathrm{ES}_{0} \stackrel{\kappa_{3}}{\longrightarrow} \mathrm{S}_{1}+\mathrm{E}, \quad \mathrm{S}_{1}+\mathrm{F} \underset{\kappa_{5}}{\stackrel{\kappa_{4}}{\rightleftharpoons}} \mathrm{FS}_{1} \stackrel{\kappa_{6}}{\longrightarrow} \mathrm{S}_{0}+\mathrm{F} \\
\mathrm{P}_{0}+\mathrm{S}_{1} \underset{\kappa_{7}}{\stackrel{\kappa_{8}}{\rightleftharpoons}} \mathrm{S}_{1} \mathrm{P}_{0} \stackrel{\kappa_{9}}{\longrightarrow} \mathrm{P}_{1}+\mathrm{S}_{1}, \quad \mathrm{P}_{1}+\mathrm{F} \underset{\kappa_{11}}{\stackrel{\kappa_{10}}{\rightleftharpoons}} \mathrm{FP}_{1} \stackrel{\kappa_{12}}{\longrightarrow} \mathrm{P}_{0}+\mathrm{F}
\end{aligned}
$$

This network describes a "cascade motif" with two layers; each layer is a "one-site modification cycle", and the same phosphatase $(F)$ acts in each layer [14, Figure 1(k)].

The network has $s=10$ species:

$$
\begin{array}{lllll}
X_{1}=\mathrm{S}_{0}, & X_{3}=\mathrm{S}_{1}, & X_{5}=\mathrm{P}_{0}, & X_{7}=\mathrm{P}_{1}, & X_{9}=\mathrm{E}, \\
X_{2}=\mathrm{ES}_{0}, & X_{4}=\mathrm{FS}_{1}, & X_{6}=\mathrm{S}_{1} \mathrm{P}_{0}, & X_{8}=\mathrm{FP}_{1}, & X_{10}=\mathrm{F} .
\end{array}
$$


There are $d=4$ conservation laws, which arise from the total amounts of substrate $S$, enzyme $E$, enzyme $F$, and product $P$, respectively:

$$
x_{1}+x_{2}+x_{3}+x_{4}+x_{6}=c_{1}, x_{2}+x_{9}=c_{2}, x_{4}+x_{8}+x_{10}=c_{3}, x_{5}+x_{7}+x_{6}+x_{8}=c_{4} .
$$

The resulting augmented system is

$$
\begin{aligned}
f_{c, \kappa, 1} & =x_{1}+x_{2}+x_{3}+x_{4}+x_{6}-c_{1}, \\
f_{c, \kappa, 2} & =x_{2}+x_{9}-c_{2}, \\
f_{c, \kappa, 3} & =\kappa_{3} x_{2}-\kappa_{4} x_{3} x_{10}+\kappa_{5} x_{4}-\kappa_{7} x_{3} x_{5}+\kappa_{8} x_{6}+\kappa_{9} x_{6}, \\
f_{c, \kappa, 4} & =x_{4}+x_{8}+x_{10}-c_{3}, \\
f_{c, \kappa, 5} & =x_{5}+x_{7}+x_{6}+x_{8}-c_{4}, \\
f_{c, \kappa, 6} & =\kappa_{7} x_{3} x_{5}-\kappa_{8} x_{6}-\kappa_{9} x_{6}, \\
f_{c, \kappa, 7} & =-\kappa_{10} x_{7} x_{10}+\kappa_{11} x_{8}+\kappa_{9} x_{6}, \\
f_{c, \kappa, 8} & =\kappa_{10} x_{7} x_{10}-\kappa_{11} x_{8}-\kappa_{12} x_{8}, \\
f_{c, \kappa, 9} & =-\kappa_{1} x_{1} x_{9}+\kappa_{2} x_{2}+\kappa_{3} x_{2}, \\
f_{c, \kappa, 10} & =-\kappa_{4} x_{3} x_{10}+\kappa_{5} x_{4}+\kappa_{6} x_{4}-\kappa_{10} x_{7} x_{10}+\kappa_{11} x_{8}+\kappa_{12} x_{8} .
\end{aligned}
$$

Consider the following upper-triangular matrix:

$$
M(\kappa)=\left(\begin{array}{cccccc}
\frac{1}{\kappa_{3}} & \frac{1}{\kappa_{3}} & 0 & -\frac{1}{\kappa_{3}} & 0 & -\frac{1}{\kappa_{3}} \\
0 & \frac{1}{\kappa_{7}} & 0 & 0 & 0 & 0 \\
0 & 0 & \frac{1}{\kappa_{9}} & \frac{1}{\kappa_{9}} & 0 & 0 \\
0 & 0 & 0 & \frac{1}{\kappa_{10}} & 0 & 0 \\
0 & 0 & 0 & 0 & \frac{1}{\kappa_{1}} & 0 \\
0 & 0 & 0 & 0 & 0 & \frac{1}{\kappa_{4}}
\end{array}\right) .
$$

Note that $\operatorname{det} M(\kappa)>0$ for all $\kappa \in \mathbb{R}_{>0}^{12}$. Following (8)-(10), the resulting system is:

$$
\begin{array}{lll}
h_{c, a, 1}=x_{1}+x_{2}+x_{3}+x_{4}+x_{6}-c_{1}, & h_{c, a, 2}=x_{2}+x_{9}-c_{2}, & h_{c, a, 3}=x_{2}-a_{1} x_{4}, \\
h_{c, a, 4}=x_{4}+x_{8}+x_{10}-c_{3}, & h_{c, a, 5}=x_{5}+x_{7}+x_{6}+x_{8}-c_{4}, & h_{c, a, 6}=x_{3} x_{5}-a_{2} x_{6}, \\
h_{c, a, 7}=x_{6}-a_{3} x_{8}, & h_{c, a, 8}=x_{7} x_{10}-a_{4} x_{8}, & h_{c, a, 9}=-x_{1} x_{9}+a_{5} x_{2}, \\
h_{c, a, 10}=-x_{3} x_{10}+a_{6} x_{4} & &
\end{array}
$$

where the effective parameters are as follows:

$$
\bar{a}_{1}=\frac{\kappa_{6}}{\kappa_{3}}, \quad \bar{a}_{2}=\frac{\kappa_{8}+\kappa_{9}}{\kappa_{7}}, \quad \bar{a}_{3}=\frac{\kappa_{12}}{\kappa_{9}}, \quad \bar{a}_{4}=\frac{\kappa_{11}+\kappa_{12}}{\kappa_{10}}, \quad \bar{a}_{5}=\frac{\kappa_{2}+\kappa_{3}}{\kappa_{1}}, \quad \bar{a}_{6}=\frac{\kappa_{5}+\kappa_{6}}{\kappa_{4}} .
$$

The associated map $\mathbb{R}_{>0}^{12} \rightarrow \mathbb{R}_{>0}^{6}$ given by $\kappa \mapsto \bar{a}(\kappa)$ is surjective.

Note that the non-conservation-law equations in $h_{c, a}$ are binomials, so the network is linearly binomial. Hence, as in (19), we use the binomial equations $h_{c, a, 3}=h_{c, a, 6}=h_{c, a, 7}=h_{c, a, 8}=h_{c, a, 9}=$ $h_{c, a, 10}=0$ to solve for the $a_{k}$ 's:

$$
a_{1}=\frac{x_{2}}{x_{4}}, \quad a_{2}=\frac{x_{3} x_{5}}{x_{6}}, \quad a_{3}=\frac{x_{6}}{x_{8}}, \quad a_{4}=\frac{x_{7} x_{10}}{x_{8}}, \quad a_{5}=\frac{x_{1} x_{9}}{x_{2}}, \quad a_{6}=\frac{x_{3} x_{10}}{x_{4}} .
$$

Substituting (25) into det $\operatorname{Jac}\left(h_{c, a}\right)$ - that is, considering the parametrization $x \mapsto\left(\phi_{a}(x), x\right)$ where $\phi_{a}(x)$ is given by 25 - yields the following critical function $C(x)$, as in 20):

$$
C(x)=\frac{x_{3} x_{10}}{x_{4} x_{8}} B(x),
$$

where $B(x)$ is the following critical polynomial: 


$$
\begin{gathered}
x_{1} x_{10} x_{2} x_{5}+x_{1} x_{10} x_{2} x_{6}+x_{1} x_{10} x_{2} x_{7}+x_{1} x_{10} x_{2} x_{8}+x_{1} x_{10} x_{5} x_{9}+x_{1} x_{10} x_{6} x_{9}+x_{1} x_{10} x_{7} x_{9}+ \\
x_{1} x_{10} x_{8} x_{9}-x_{1} x_{2} x_{5} x_{8}+x_{1} x_{2} x_{7} x_{8}-x_{1} x_{5} x_{8} x_{9}+x_{1} x_{7} x_{8} x_{9}+x_{10} x_{2} x_{5} x_{9}+x_{10} x_{2} x_{6} x_{9}+x_{10} x_{2} x_{7} x_{9}+ \\
x_{10} x_{2} x_{8} x_{9}+x_{10} x_{3} x_{5} x_{9}+x_{10} x_{3} x_{6} x_{9}+x_{10} x_{3} x_{7} x_{9}+x_{10} x_{3} x_{8} x_{9}+x_{10} x_{4} x_{5} x_{9}+x_{10} x_{4} x_{6} x_{9}+ \\
x_{10} x_{4} x_{7} x_{9}+x_{10} x_{4} x_{8} x_{9}+x_{10} x_{5} x_{6} x_{9}-x_{2} x_{5} x_{8} x_{9}+x_{2} x_{7} x_{8} x_{9}+x_{3} x_{4} x_{5} x_{9}+x_{3} x_{4} x_{6} x_{9}+x_{3} x_{4} x_{7} x_{9}+ \\
x_{3} x_{4} x_{8} x_{9}+x_{3} x_{7} x_{8} x_{9}+x_{4} x_{5} x_{6} x_{9}-x_{4} x_{5} x_{8} x_{9}-x_{4} x_{6} x_{7} x_{9}+x_{4} x_{7} x_{8} x_{9} .
\end{gathered}
$$

Consistent with Lemma 4.5. $B(x)$ is homogeneous with total degree $d=4$ and square-free monomials.

From the conservation laws (23), we see that this network is conservative and hence dissipative. It is also straightforward to check (for instance, using criteria in [42, 35]) that it has no boundary steady states. Thus, we can follow Procedure 4.7 to find a witness as follows:

Step 1. We compute the following sign:

$$
(-1)^{\operatorname{rank}(N)+1}=(-1)^{s-d+1}=(-1)^{10-4+1}=-1 .
$$

We see that $B(x)$ has five (underlined) terms with the above negative sign; one such term is:

$$
-x_{1} x_{2} x_{5} x_{8} .
$$

Accordingly, define $x^{*} \in \mathbb{R}^{10}$ with coordinates $\lambda$ (in indices $1,2,5$, and 8) and 1 (all others):

$$
x^{*}=(\lambda, \lambda, 1,1, \lambda, 1,1, \lambda, 1,1) .
$$

Then we have

$$
B\left(x^{*}\right)=-\lambda^{4}+\lambda^{3}+7 \lambda^{2}+14 \lambda+5 .
$$

It follows that $B\left(x^{*}\right)<0$ if $\lambda$ is larger than the largest positive root of the polynomial (27). There are many well-known upper bounds for the real roots of a univariate polynomial. Here, we use an elementary bound, the sum of the absolute values of all coefficients:

$$
1+1+7+14+5=28 \text {. }
$$

Let $\lambda=29$; then, $\left.B\left(x^{*}\right)\right|_{\lambda=29}=-676594<0$.

Step 2. To solve for $c^{*}$, we substitute $\left.x^{*}\right|_{\lambda=29}$, as in (26), into equation (23), which yields:

$$
c^{*}=(61,30,31,60)
$$

Steps 3-4. We substitute $\left.x^{*}\right|_{\lambda=29}$, as in (26), into equation (25). This yields:

$$
\text { and } \phi_{a}\left(x^{*}\right)=(29,29,1 / 29,1 / 29,1,1) \text {. }
$$

Finally, we choose $\kappa^{*}$ for which $\bar{a}\left(\kappa^{*}\right)=\phi_{a}\left(x^{*}\right)$, as in (24):

$$
\kappa^{*}=(2,1,1,30,1,29,1,28,1,2,1 / 29,1 / 29) .
$$

So, $\left(\kappa^{*}, c^{*}\right)$ is a witness to multistationarity.

4.2. Open multistationarity regions in parameter space. We saw in Procedure 4.7 that, for linearly binomial networks, finding $x^{*}$ with $\operatorname{sign}\left(B\left(x^{*}\right)\right)=(-1)^{\operatorname{rank}(N)+1}$ allows us to obtain a witness to multistationarity $\left(\kappa^{*}, c^{*}\right)$ satisfying $\varphi\left(\kappa^{*}, c^{*}\right)=\psi\left(x^{*}\right)$ for the maps:

$$
\begin{aligned}
& \mathbb{R}_{>0}^{s} \stackrel{\psi}{\rightarrow} \mathbb{R}_{>0}^{s-d} \times \mathbb{R}_{>0}^{d} \stackrel{\varphi}{\leftarrow} \mathbb{R}_{>0}^{m} \times \mathbb{R}_{>0}^{d} \\
& x \mapsto \quad(a, c) \quad \longleftrightarrow(\kappa, c),
\end{aligned}
$$


where $\psi: x \mapsto(a, c)$ is given by:

$$
\begin{cases}a_{k}=\psi_{k}=x^{\gamma_{k}-\delta_{k}}, & k=1,2, \ldots, s-d \\ c_{k}=\psi_{s-d+k}=(W x)_{k}, & k=1,2, \ldots, d\end{cases}
$$

and $\varphi$ is given by

$$
\varphi(\kappa, c):=(\bar{a}(\kappa), c) .
$$

Therefore, the region in the parameter space $\mathbb{R}_{>0}^{m} \times \mathbb{R}_{>0}^{d}$ of the $(\kappa, c)$ 's where degree theory guarantees multistationarity is

$$
\varphi^{-1}(\psi(U))
$$

where

$$
U=\left\{x \in \mathbb{R}_{>0}^{s} \mid \operatorname{sign}(B(x))=(-1)^{\operatorname{rank}(N)+1}\right\} .
$$

We will show that the set $\psi(U)$ is open (Theorem 4.10), and thus so is $\varphi^{-1}(\psi(U)$ ), our region of interest (Corollary 4.11). For "typical" networks, the multistationarity regions of parameter space are full-dimensional. The interpretation for applications is that multistationarity persists under small perturbations of the rate constants and the total-constant values (equivalently, initial values). Such robustness properties are desirable in biological systems. In our setting, it is not immediately evident that we find an open region in the parameter space of the variables $(c, \kappa)$ because we detect a multistationarity region in the $x$ space and not in the parameter space.

Before stating the results in this section, we show in Figure 2 a "slice" of the set $U$, as in (31), arising from the network in Example 4.8. More precisely, starting with the critical polynomial $B$ from that example, we display the (open) region where $B<0$, under the following specialization:

$$
x_{3}=x_{4}=1, \quad x_{5}=x_{8}=6, \quad x_{6}=x_{7}=1, \quad x_{9}=x_{10}=1 .
$$

This region, in $\left(x_{1}, x_{2}\right)$-space, is guaranteed by degree theory to yield multistationarity.

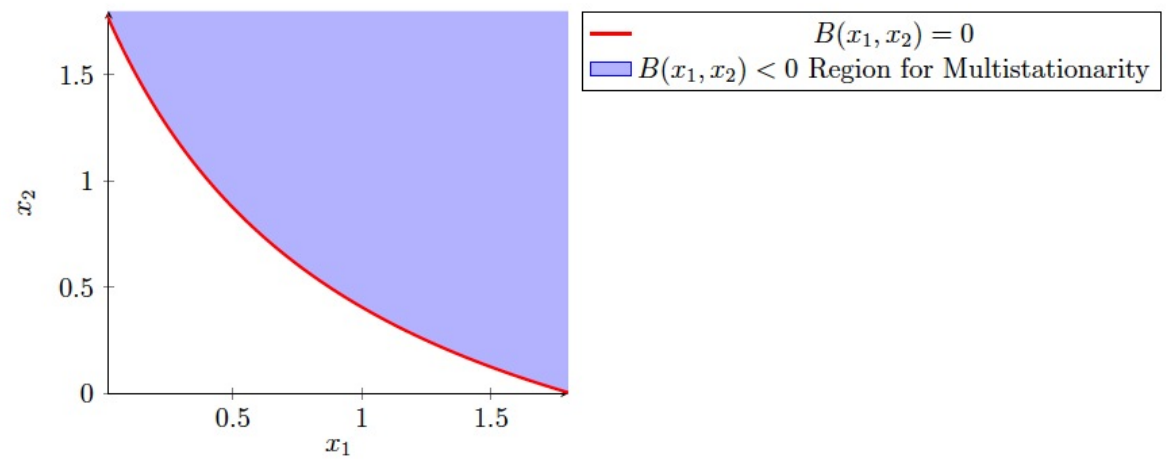

Figure 2. The shaded region depicts a "slice" of the multistationarity region $U$, as in (31), for the network in Example 4.8. The slice arises from the specialization (32). 
Lemma 4.9. Let $G$ be a linearly binomial network with critical polynomial $B(x)$ and map $\psi$ as in (28). Then for every $x^{*} \in \mathbb{R}_{>0}^{s}$, we have $\left.\operatorname{det} \mathrm{Jac} \psi\right|_{x=x^{*}} \neq 0$ if and only if $B\left(x^{*}\right) \neq 0$.

Proof. It is straightforward to verify, using equations (18) and (28), the following equality:

$$
\operatorname{Jac} \psi= \pm\left.\left(\frac{1}{\Pi_{k=1}^{s-d} x^{\delta_{k}}}\right) \cdot \operatorname{Jac}\left(h_{c, a}\right)\right|_{a_{k}=x^{\gamma_{k}-\delta_{k}}}, \quad k=1,2, \ldots, s-d .
$$

Thus, for every $y \in \mathbb{R}_{>0}^{s}$ we obtain the first equality here:

$\left.\operatorname{det} \operatorname{Jac} \psi\right|_{x=y}= \pm\left.\left(\frac{1}{\Pi_{k=1}^{s-d} y^{\delta_{k}}}\right) \cdot\left(\left.\operatorname{det}\left(\operatorname{Jac}\left(h_{c, a}\right)\right)\right|_{a_{k}=x^{\gamma_{k}-\delta_{k}}}\right)\right|_{x=y}= \pm \frac{C(y)}{\Pi_{k=1}^{s-d} y^{\delta_{k}}}= \pm \frac{y^{\alpha}}{y^{\beta}} \cdot \frac{B(y)}{\Pi_{k=1}^{s-d} y^{\delta_{k}}}$,

and the second and third equalities arise, respectively, from equation $(20)$ and Lemma 4.4 . Thus, for every $y \in \mathbb{R}_{>0}^{s}$, we have $\left.\operatorname{det} \operatorname{Jac} \psi\right|_{x=y} \neq 0$ if and only if $B(y) \neq 0$.

Theorem 4.10. Let $G$ be a linearly binomial network with critical polynomial $B(x)$ and map $\psi$ as in (28). Let $U$ be as in (31). Then $\psi(U)$ is an open set in the $(c, a)$-space $\mathbb{R}_{>0}^{s}$.

Proof. Note that $B\left(x^{*}\right) \neq 0$ for any $x^{*} \in U$. So, we deduce from Lemma 4.9 that the Jacobian of $\psi$ never vanishes on $U$. The result now follows from the Inverse Function Theorem.

Corollary 4.11. Let $G$ be a linearly binomial network, with maps $\psi$ and $\varphi$ as in (28)-(29). Then the multistationarity region of parameter space $\varphi^{-1}(\psi(U))$ as in $(30)$, is an open set in the $(\kappa, c)$ space $\mathbb{R}_{>0}^{m} \times \mathbb{R}_{>0}^{d}$.

Proof. The map $\varphi$ is continuous, and, by Theorem 4.10, $\psi(U)$ is open. Hence, $\varphi^{-1}(\psi(U))$ is open.

\section{Many MESSI NeTWORKS ARE Linearly BinOMial NETWORKS}

In this section, we show that a class of networks that includes many biological signaling networks are linearly binomial networks (Theorem 5.3). These networks are so-called MESSI networks [35], whose definition we recall below.

5.1. Definition of MESSI systems. A chemical reaction network with species set $\mathscr{S}$ is a MESSI network if there is a partition

$$
\mathscr{S}=\mathscr{S}^{(0)} \bigsqcup \mathscr{S}^{(1)} \bigsqcup \mathscr{S}^{(2)} \bigsqcup \cdots \bigsqcup \mathscr{S}^{(m)},
$$

where $m \geq 1$ and $\bigsqcup$ denotes disjoint union, such that the complexes and reactions satisfy the conditions below. Species in $\mathscr{S}^{(0)}$ and $\mathscr{S}_{1}:=\mathscr{S} \backslash \mathscr{S}^{(0)}$ are called, respectively, intermediate and core. Complexes are also partitioned into two disjoint sets: intermediate complexes and core complexes [15]. Each intermediate complex consists of a unique intermediate species.

Core complexes satisfy the following two conditions:

(i) They are molecular or bimolecular and consist of either one or two core species.

(ii) If the core complex consists of two species $X_{i}, X_{j}$, they must belong to different sets $\mathscr{S}^{(\alpha)}, \mathscr{S}^{(\beta)}$ (with $\alpha \neq \beta$ and $\alpha, \beta \geq 1$ ). 
We say that complex $y$ reacts to complex $y^{\prime}$ via intermediates if either $y \rightarrow y^{\prime}$ or there exists a path of reactions from $y$ to $y^{\prime}$ only through intermediate complexes. This is denoted by $y \rightarrow \circ y^{\prime}$. The intermediate complexes of a MESSI network satisfy moreover the following condition. For every intermediate complex $y$, there exist core complexes $y^{\prime}$ and $y^{\prime \prime}$ such that $y^{\prime} \rightarrow_{\circ} y$ and $y \rightarrow \circ y^{\prime \prime}$.

The reactions of MESSI networks are constrained by the following rules:

(i) If three species are related by $X_{i}+X_{j} \rightarrow_{\circ} X_{k}$ or $X_{k} \rightarrow_{\circ} X_{i}+X_{j}$, then $X_{k}$ is an intermediate species.

(ii) If two core species $X_{i}, X_{j}$ are related by $X_{i} \rightarrow_{\circ} X_{j}$, then there exists $\alpha \geq 1$ such that both belong to $\mathscr{S}^{(\alpha)}$.

(iii) If $X_{i}+X_{j} \rightarrow \circ X_{k}+X_{\ell}$, then there exist $\alpha \neq \beta$ such that $X_{i}, X_{k} \in \mathscr{S}^{(\alpha)}, X_{j}, X_{\ell} \in \mathscr{S}^{(\beta)}$ or $X_{i}, X_{\ell} \in \mathscr{S}^{(\alpha)}, X_{j}, X_{k} \in \mathscr{S}^{(\beta)}$.

The partition (33) defines a MESSI structure on the network. A MESSI system is the mass-action kinetics dynamical system (1) associated with a MESSI network.

5.2. The associated digraphs. We now present the digraphs $G_{1}, G_{2}^{\circ}$, and $G_{E}$ associated to a MESSI network $G$ through an example. For the actual definition we refer the reader to [35].

Example 5.1 (Two-layer cascade, continued). Recall the two-layer cascade network in Example 4.8. We consider the following partition $\mathscr{S}^{(0)}=\left\{E S_{0}, F S_{1}, S_{1} P_{0}, F P_{1}\right\}$ (intermediate species), and $\mathscr{S}^{(1)}=\left\{S_{0}, S_{1}\right\}, \mathscr{S}^{(2)}=\left\{P_{0}, P_{1}\right\}, \mathscr{S}^{(3)}=\{E\}, \mathscr{S}^{(4)}=\{F\}$ (partition of the core species). The intermediate complexes correspond to the intermediate species, and the remaining complexes are core complexes. This partition defines a MESSI structure in the network.

We first define a digraph $G_{1}$ by keeping the core complexes as vertices and considering the edges $y \rightarrow y^{\prime}$ if $y \rightarrow_{\circ} y^{\prime}$ in $G$. The labels assigned to these edges, $\boldsymbol{\tau}(\kappa)$, are rational functions of the original rate constants $\kappa$, following [15, Theorem 3.1]:

$$
\begin{aligned}
\boldsymbol{\tau}: \mathbb{R}_{>0}^{m} & \rightarrow \mathbb{R}_{>0}^{r} \\
\kappa & \mapsto\left(\tau_{1}(\kappa), \tau_{2}(\kappa), \ldots, \tau_{r}(\kappa)\right),
\end{aligned}
$$

where $r$ denotes the number of edges in $G_{1}$. We then define a new digraph $G_{2}$ where we "hide" the concentrations of some of the species in the labels. We keep all monomolecular reactions $X_{i} \rightarrow X_{j}$ and for each reaction $X_{i}+X_{\ell} \stackrel{\tau}{\longrightarrow} X_{j}+X_{m}$, with $X_{i}, X_{j} \in \mathscr{S}^{(\alpha)}, X_{\ell}, X_{m} \in \mathscr{S}^{(\beta)}$, we consider two reactions $X_{i} \stackrel{\tau x_{\ell}}{\longrightarrow} X_{j}$ and $X_{\ell} \stackrel{\tau x_{i}}{\longrightarrow} X_{m}$. We obtain a multidigraph $M G_{2}$ that may contain loops or parallel edges between some pairs of nodes (i.e., directed edges with the same source and target nodes). We define the digraph $G_{2}$ by collapsing into one edge all parallel edges in $M G_{2}$ and we define the labels of each edge as the sum of the labels of the corresponding collapsed edges in $M G_{2}$. Note that these labels might depend on some of the concentrations. We will moreover denote by $G_{2}^{\circ}$ the digraph obtained from $G_{2}$ by deleting loops and isolated nodes. We finally define the associated digraph $G_{E}$. The set of vertices of $G_{E}$ equals $\left\{\mathscr{S}^{(\alpha)} \mid \alpha \geq 1\right\}$. The pair $\left(\mathscr{S}^{(\alpha)}, \mathscr{S}^{(\beta)}\right)$ is an edge of $G_{E}$ when there is a species in $\mathscr{S}^{(\alpha)}$ in a label of an edge in $G_{2}^{\circ}$ between (distinct) species of $\mathscr{S}^{(\beta)}$.

The graphs $G_{1}, G_{2}^{\circ}$, and $G_{E}$ associated to this network are the following: 


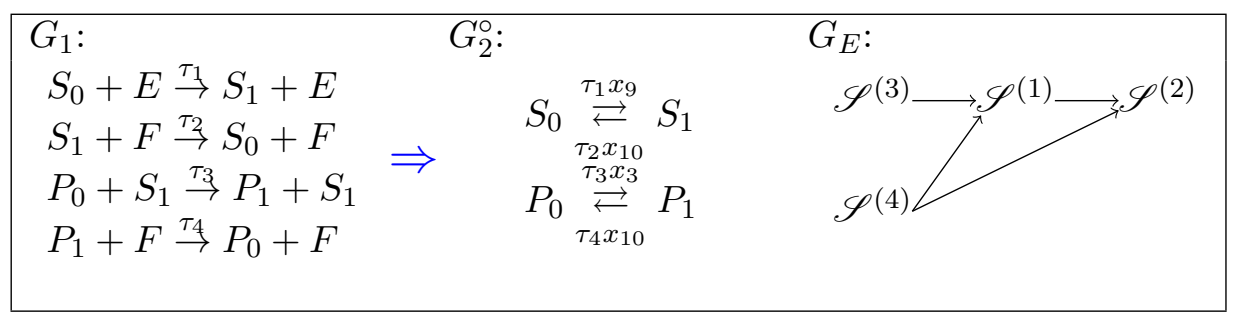

Here, as defined in (34), $\boldsymbol{\tau}: \mathbb{R}_{>0}^{12} \rightarrow \mathbb{R}_{>0}^{4}$ is

$$
\tau_{1}=\frac{\kappa_{1}}{\kappa_{2}+\kappa_{3}}, \quad \tau_{2}=\frac{\kappa_{4}}{\kappa_{5}+\kappa_{6}}, \quad \tau_{3}=\frac{\kappa_{7}}{\kappa_{8}+\kappa_{9}}, \quad \tau_{4}=\frac{\kappa_{10}}{\kappa_{11}+\kappa_{12}} .
$$

Remark 5.2. An important fact is that for any MESSI network with digraph $G$, once the edges in $G_{1}$ are labeled with the constants $\boldsymbol{\tau}(\kappa)$, the steady states of the mass-action chemical reaction system defined by $G$ and those of $G_{1}$ are in one-to-one correspondence. Moreover, $G_{1}$ and $G_{2}^{\circ}$, together with the corresponding equations of the intermediate species, define the whole variety of steady states of $G$.

5.3. Main result: conditions for MESSI linearly binomial networks. In this section we give sufficient conditions on a MESSI system that ensure that the network is linearly binomial (Definition 3.4). We define a further condition $(\mathcal{C})$ : For every intermediate complex $y$ there exists a unique core complex $y^{\prime}$ such that $y^{\prime} \rightarrow \circ y$ in $G$.

Theorem 5.3. Let $G$ be the underlying digraph of a MESSI system with $m$ reactions ( $m$ directed edges). Assume that $G$ satisfies condition $(\mathcal{C})$ and that the associated digraph $G_{E}$ has no directed cycles, the underlying undirected graph of the associated graph $G_{2}^{\circ}$ is a forest (an acyclic graph), and $M G_{2}$ has no parallel edges. Then, there exist:

(1) $\bar{a}_{1}(\kappa), \bar{a}_{2}(\kappa), \ldots, \bar{a}_{\bar{m}}(\kappa) \in \mathbb{Q}(\kappa)$ such that $\bar{a}_{i}\left(\kappa^{*}\right)$ is defined and, moreover, $\bar{a}_{i}\left(\kappa^{*}\right)>0$ for every $i=1,2, \ldots, \bar{m}$ and for all $\kappa^{*} \in \mathbb{R}_{>0}^{m}$, and

(2) an $(s-d) \times(s-d)$ matrix $M(\kappa)$ with $\operatorname{det} M\left(\kappa^{*}\right)>0$ for all $\kappa^{*} \in \mathbb{R}_{>0}^{m}$,

such that the functions

$$
\left(\bar{h}_{j_{1}}, \bar{h}_{j_{2}}, \ldots, \bar{h}_{j_{s-d}}\right)^{\top}:=\quad M(\kappa)\left(f_{j_{1}}, f_{j_{2}}, \ldots, f_{j_{s-d}}\right)^{\top}
$$

are binomials, where $f$ is the polynomial system obtained from the ODEs (1) of the network $G$, and every nonconstant coefficient in $\bar{h}_{j_{l}}$ is equal to a rational-number multiple of some $\bar{a}_{i}(\kappa)$. Therefore, if the map $\bar{a}$ is surjective, then $\bar{a}$ is a reparametrization map, as in (7), and $\bar{h}_{j_{1}}, \ldots, \bar{h}_{j_{s-d}}$ is an effective steady-state function $h_{c, a}(x)$ of $G$, as in (9)-(10), and so $G$ is a linearly binomial network.

We prove Theorem 5.3 in Appendix A.

Remark 5.4. The extra hypothesis in Theorem 5.3 that guarantees that $G$ is a linearly binomial network - namely, the condition that the map $\bar{a}$ is surjective - holds for every example we have examined. See, for instance, Examples 3.19 and 4.8 .

Example 5.5 (Two-layer cascade, continued). Recall the two-layer cascade network in Examples 4.8 and 5.1 . Notice that $G$ satisfies condition $(\mathcal{C})$ and that the associated digraph $G_{E}$ has no directed cycles (see Example 5.1). Moreover, the underlying undirected graph of the associated graph $G_{2}^{\circ}$ is a forest:

$$
S_{0}-S_{1} \quad P_{0}-P_{1}
$$


and $M G_{2}$ has no parallel edges. Hence, Theorem 5.3 applies, and in fact the resulting binomials are the ones we computed in Example 4.8 .

\section{EStablishing MULTistationarity USING TRIANGULAR FORMS}

For networks that are non-dissipative or have boundary steady states, Theorems 3.12 and 4.6 do not apply. Accordingly, in this section, we propose a method to analyze such networks. The main idea is to find a degenerate positive steady state $x^{*}$ and then perturb the corresponding parameters. The hope is that $x^{*}$ will break into two distinct positive steady states. We prove that this approach will succeed under certain conditions (Theorem 6.5). Specifically, we require that the steady-state equations admit a "triangular form" (Definition 6.2). Finally, we show that such a triangular form exists whenever the steady-state equations form a "general zero-dimensional system" (Corollary 6.12).

Before stating our results, we introduce our running example, which we first show has boundary steady states (so, our earlier results do not apply).

Example 6.1 (Calvin cycle). Consider the following "elementary mode" of the Calvin cycle network proposed in [23, Fig. 4]:

$$
\begin{array}{ll}
\mathrm{RuBP}+\mathrm{E}_{1} \stackrel{\kappa_{1}}{\longrightarrow} \mathrm{RuBPE}_{1} \stackrel{\kappa_{2}}{\longrightarrow} 2 \cdot \mathrm{PGA}+\mathrm{E}_{1}, & \mathrm{PGA}+\mathrm{E}_{2} \stackrel{\kappa_{3}}{\longrightarrow} \mathrm{PGAE}_{2} \stackrel{\kappa_{4}}{\longrightarrow} \mathrm{DPGA}+\mathrm{E}_{2}, \\
\mathrm{DPGA}+\mathrm{E}_{3} \stackrel{\kappa_{5}}{\longrightarrow} \mathrm{DPGAE}_{3} \stackrel{\kappa_{6}}{\longrightarrow} \mathrm{GAP}+\mathrm{E}_{3}, & 5 \cdot \mathrm{GAP}+\mathrm{E}_{4} \stackrel{\kappa_{7}}{\longrightarrow} \mathrm{GPAE}_{4} \stackrel{\kappa_{8}}{\longrightarrow} 3 \cdot \mathrm{Ru}_{5} \mathrm{P}+\mathrm{E}_{4}, \\
\mathrm{Ru}_{5} \mathrm{P}+\mathrm{E}_{5} \stackrel{\kappa_{9}}{\longrightarrow} \mathrm{Ru}_{5} \mathrm{PE}_{5} \stackrel{\kappa_{10}}{\longrightarrow} \mathrm{RuBP}+\mathrm{E}_{5}, & \mathrm{GAP}+\mathrm{E}_{7} \stackrel{\kappa_{11}}{\longrightarrow} \mathrm{GAPE}_{7} \stackrel{\kappa_{12}}{\longrightarrow} \mathrm{E}_{7} .
\end{array}
$$

This network is obtained by shutting down 9 transporter reactions from the original Calvin cycle network (see " $v_{1}^{E M}$ " in [23, page 218]). Let

$$
\begin{array}{llllll}
X_{1}=\mathrm{RuBP}, & X_{2}=\mathrm{E}_{1}, & X_{3}=\mathrm{RuBPE}_{1}, & X_{4}=\mathrm{PGA}, & X_{5}=\mathrm{E}_{2}, & X_{6}=\mathrm{PAGE}_{2}, \\
X_{7}=\mathrm{DPGA}, & X_{8}=\mathrm{E}_{3}, & X_{9}=\mathrm{DPGAE}_{3}, & X_{10}=\mathrm{GAP}, & X_{11}=\mathrm{E}_{4}, & X_{12}=\mathrm{GAPE}_{4}, \\
X_{13}=\mathrm{Ru}_{5} \mathrm{P}, & X_{14}=\mathrm{E}_{5}, & X_{15}=\mathrm{Ru}_{5} \mathrm{PE}_{5}, & X_{16}=\mathrm{E}_{7}, & X_{17}=\mathrm{GAPE}_{7} . &
\end{array}
$$

The function $f_{c, \kappa}(x)$ is

$$
\begin{array}{ll}
f_{c, \kappa, 1}=-\kappa_{1} x_{1} x_{2}+\kappa_{10} x_{15}, & f_{c, \kappa, 2}=x_{2}+x_{3}-c_{1} \\
f_{c, \kappa, 3}=\kappa_{1} x_{1} x_{2}-\kappa_{2} x_{3}, & f_{c, \kappa, 4}=2 \kappa_{2} x_{3}-\kappa_{3} x_{4} x_{5}, \\
f_{c, \kappa, 5}=x_{5}+x_{6}-c_{2}, & f_{c, \kappa, 6}=\kappa_{3} x_{4} x_{5}-\kappa_{4} x_{6} \\
f_{c, \kappa, 7}=\kappa_{4} x_{6}-\kappa_{5} x_{7} x_{8}, & f_{c, \kappa, 8}=x_{8}+x_{9}-c_{3}, \\
f_{c, \kappa, 9}=\kappa_{5} x_{7} x_{8}-\kappa_{6} x_{9}, & f_{c, \kappa, 10}=\kappa_{6} x_{9}-5 \kappa_{7} x_{10}^{5} x_{11}-\kappa_{11} x_{10} x_{16}, \\
f_{c, \kappa, 11}=x_{11}+x_{12}-c_{4}, & f_{c, \kappa, 12}=\kappa_{7} x_{10}^{5} x_{11}-\kappa_{8} x_{12}, \\
f_{c, \kappa, 31}=3 \kappa_{8} x_{12}-\kappa_{9} x_{13} x_{14}, & f_{c, \kappa, 14}=x_{14}+x_{15}-c_{5}, \\
f_{c, \kappa, 15}=\kappa_{9} x_{13} x_{14}-\kappa_{10} x_{15}, & f_{c, \kappa, 16}=x_{16}+x_{17}-c_{6}, \\
f_{c, \kappa, 17}=\kappa_{11} x_{10} x_{16}-\kappa_{12} x_{17} . &
\end{array}
$$

If we set $x_{1}=x_{3}=x_{4}=x_{6}=x_{7}=x_{9}=x_{10}=x_{12}=x_{13}=x_{15}=x_{17}=0$, the polynomials $f_{c, \kappa, i}$ (for $i=1,2, \ldots, 17$ ) above become

$$
0, x_{2}-c_{1}, 0,0, x_{5}-c_{2}, 0,0, x_{8}-c_{3}, 0,0, x_{11}-c_{4}, 0,0, x_{14}-c_{5}, 0, x_{16}-c_{6}, 0 .
$$

Thus, for any rate-constant vector $\kappa$ and for any total-constant vector $c$, we have the following boundary steady state:

$$
\left(0, c_{1}, 0,0, c_{2}, 0,0, c_{3}, 0,0, c_{4}, 0,0, c_{5}, 0, c_{6}, 0\right)
$$


Thus, Theorem 3.12 does not apply to the Calvin cycle network.

6.1. Steady-state equations that admit a triangular form. In this section, we investigate multistationarity for networks whose steady-state equations admit a triangular form (Theorem 6.5).

Definition 6.2. Let $G$ be a reaction network with $s$ species. The steady-state equations of $G$ admit a triangular form if there exists a system $h_{c, a}(x)=0 \sqrt{10}$ for $G$ and there exist functions $T_{1}, T_{2}, \ldots, T_{s}$ of the following form:

$$
\begin{aligned}
T_{s} & =\theta_{s}\left(c, a, x_{s}\right) \\
T_{s-1} & =x_{s-1}-\theta_{s-1}\left(c, a, x_{s}\right) \\
T_{s-2} & =x_{s-2}-\theta_{s-2}\left(c, a, x_{s-1}, x_{s}\right) \\
& \vdots \\
T_{1} & =x_{1}-\theta_{1}\left(c, a, x_{2}, \ldots, x_{s}\right)
\end{aligned}
$$

such that:

(i) each $\theta_{i}: \mathbb{R}_{>0}^{d} \times \mathbb{R}_{>0}^{\bar{m}} \times \mathbb{R}_{>0}^{s-1} \rightarrow \mathbb{R}$ is a $C^{2}$-function, $\theta_{s}$ does not depend on $x_{1}, x_{2}, \ldots, x_{s-1}$, and (for $1 \leq i \leq s-1$ ) $\theta_{i}$ does not depend on $x_{1}, x_{2}, \ldots, x_{i}$; and

(ii) there exists a variety $\mathcal{W} \subsetneq \mathbb{C}^{d} \times \mathbb{C}^{\bar{m}}$ such that for all $\left(c^{*}, a^{*}\right) \in \mathbb{R}_{>0}^{d} \times \mathbb{R}_{>0}^{\bar{m}} \backslash \mathcal{W}$, the positive zeros of $h_{c^{*}, a^{*}}$ coincide with the positive zeros of the system

$$
T_{s}\left(c^{*}, a^{*}, x_{s}\right), \ldots, T_{1}\left(c^{*}, a^{*}, x_{1}, x_{2}, \ldots, x_{s}\right) .
$$

We recall the standard notion of singular point and we state a useful lemma (Lemma 6.4) that we will prove in Appendix B using the Implicit Function Theorem.

Definition 6.3. Consider a polynomial $f \in \mathbb{R}[b, z]$, where $(b, z) \in \mathbb{R}^{n} \times \mathbb{R}$. We say $\left(b^{*}, z^{*}\right) \in \mathbb{R}^{n} \times \mathbb{R}$ is a singular point of $f$ if $f\left(b^{*}, z^{*}\right)=0, \frac{\partial f}{\partial z}\left(b^{*}, z^{*}\right)=0$, and $\frac{\partial f}{\partial b_{i}}\left(b^{*}, z^{*}\right)=0$, for all $i=1, \ldots, n$. We say $\left(b^{*}, z^{*}\right)$ is a regular point of $f$ if $f\left(b^{*}, z^{*}\right)=0$ and $\left(b^{*}, z^{*}\right)$ is not a singular point of $f$. Given a univariate polynomial $f \in \mathbb{R}[z]$, we say that $z^{*} \in \mathbb{R}$ is a multiplicity-2 solution of $f(z)=0$ if $f\left(z^{*}\right)=0, \frac{d f}{d z}\left(z^{*}\right)=0$, and $\frac{d^{2} f}{d z^{2}}\left(z^{*}\right) \neq 0$.

Lemma 6.4. Consider a $C^{2}$-function $f: \mathbb{R}^{n+1} \rightarrow \mathbb{R}$. Assume that $\left(a^{*}, z^{*}\right)=\left(a_{1}^{*}, a_{2}^{*}, \ldots, a_{n}^{*}, z\right) \in$ $\mathbb{R}^{n+1}$ satisfies the following:

(1) $z^{*} \in \mathbb{R}$ is a multiplicity-2 solution of $f\left(a^{*}, z\right)=0$, and

(2) there exists an index $\ell \in[n]$ such that $\frac{\partial f}{\partial a_{\ell}}\left(a^{*}, z^{*}\right) \neq 0\left(\right.$ i.e. $\left(a^{*}, z^{*}\right)$ is a regular point of $\left.f\right)$. Then for every $\epsilon>0$, there exists $\delta>0$ such that for all $\delta^{\prime} \in(0, \delta)$, for either $a_{\ell}^{* *}=a_{\ell}^{*}-\delta^{\prime}$ or $a_{\ell}^{* *}=a_{\ell}^{*}+\delta^{\prime}$, the equation $f\left(a^{* *}, z\right)=0$, where we set $a_{i}^{* *}=a_{i}^{*}$ for all $i \neq \ell$, has two distinct real solutions $z^{(1)}$ and $z^{(2)}$, for which $\left|z^{(1)}-z^{*}\right|<\epsilon$ and $\left|z^{(2)}-z^{*}\right|<\epsilon$.

We are ready to present a result that uses the existence of a triangular form to find witnesses of multistationarity.

Theorem 6.5 (Multistationarity when steady-state equations admit a triangular form). Let $G$ be a reaction network with s species. Suppose that the steady-state equations of $G$ admit a simplified system $h_{c, a}$, a triangular form via functions $T_{1}, T_{2}, \ldots, T_{s}$, and a variety $\mathcal{W} \subsetneq \mathbb{C}^{d} \times \mathbb{C}^{\bar{m}}$ (as in Definition 6.2). Fix a total-constant vector $c^{*} \in \mathbb{R}_{>0}^{d}$, effective parameters $a^{*} \in \mathbb{R}_{>0}^{\bar{m}}$, and $x^{*} \in \mathbb{R}_{>0}^{s}$. Assume that: 
(1) $T_{1}\left(c^{*}, a^{*}, x_{2}^{*}, \ldots, x_{s}^{*}\right)=T_{2}\left(c^{*}, a^{*}, x_{3}^{*}, \ldots, x_{s}^{*}\right)=\cdots=T_{s}\left(c^{*}, a^{*}, x_{s}^{*}\right)=0$ (i.e., $x^{*}$ is a positive steady state of the system defined by $G, c^{*}$, and any $\kappa^{*}$ for which $\left.\bar{a}\left(\kappa^{*}\right)=a^{*}\right)$,

(2) $x_{s}^{*}$ is a multiplicity-2 solution of $T_{s}\left(c^{*}, a^{*}, x_{s}\right)=0$,

(3) there exists an index $i \in\{1,2, \ldots, \bar{m}\}$ such that $\left(a_{i}^{*}, x_{s}^{*}\right)$ is a regular point of

$$
T_{s}\left(c^{*} ; a_{1}^{*}, \ldots, a_{i-1}^{*}, a_{i}, a_{i+1}^{*}, \ldots, a_{\bar{m}}^{*}, x_{s}\right)=0,
$$

(4) $\left(c^{*}, a^{*}\right) \notin \mathcal{W}$.

Then $G$ is multistationary. Moreover, a witness to multistationarity is guaranteed as follows: there exists $\delta>0$ such that for all $\delta^{\prime} \in(0, \delta)$, for either $a_{i}^{* *}=a_{i}^{*}-\delta^{\prime}$ or $a_{i}^{* *}=a_{i}^{*}+\delta^{\prime}$, the mass-action system given by $G, c^{*}$, and any $\kappa^{*}$ for which $\bar{a}\left(\kappa^{*}\right)=\left(a_{1}^{*}, \ldots, a_{i-1}^{*}, a_{i}^{* *}, a_{i+1}^{*}, \ldots, a_{\bar{m}}^{*}\right)$ has at least two positive steady states.

Proof. Straightforward from Lemma 6.4 and Definition 6.2.

Remark 6.6. Theorem 6.5 suggests a procedure for finding a witness to multistationarity for networks that admit a triangular form. Namely, find a degenerate positive steady state $x^{*}$ and associated values for $c^{*}$ and $a^{*}$, and then perturb some coordinate of $a^{*}$ by a small amount. See Example 6.13. However, in practice this perturbation method could fail if we can obtain only approximations for $x^{*}, c^{*}$, and $a^{*}$, and if the multistationary region in the space of $a^{*}$ 's is too small for such approximations to find a witness. Hence, our approach is most promising when $x^{*}, c^{*}$, and $a^{*}$ can be exactly chosen with rational-number coordinates (as in Example 6.13). Indeed, for linearly binomial networks, this is easier as it is enough to find a solution $x^{*}$ of $B\left(x^{*}\right)=0$, and the critical function $B$ has the nice properties detailed in Lemma 4.4. Nevertheless, for this case, we gave other methods for obtaining witnesses to multistationarity.

6.2. Sufficient conditions for a triangular form. Theorem 6.5 gave an approach to multistationarity for networks whose steady-state equations admit a triangular form. In turn, Corollary6.12 below guarantees that a network admits such a triangular form as long as the steady-state equations form a "general zero-dimensional system".

Definition 6.7. A set of $s$ polynomials

$$
H=\left\{h_{1}, h_{2}, \ldots, h_{s}\right\} \subseteq \mathbb{C}\left[a_{1}, a_{2}, \ldots a_{n}, x_{1}, x_{2}, \ldots, x_{s}\right]=\mathbb{C}[a, x]
$$

forms a general zero-dimensional system if there exists a variety $\mathcal{W} \subsetneq \mathbb{C}^{n}$ such that for any $a^{*}=$ $\left(a_{1}^{*}, a_{2}^{*}, \ldots, a_{n}^{*}\right) \in \mathbb{C}^{n} \backslash \mathcal{W}$, the system $\left.h_{1}\right|_{a=a^{*}}=\left.h_{2}\right|_{a=a^{*}}=\ldots=\left.h_{s}\right|_{a=a^{*}}=0$ satisfies:

(A1) the number of complex solutions is finite and nonzero;

(A2) for any distinct complex solutions $x^{*}=\left(x_{1}^{*}, \ldots, x_{s}^{*}\right)$ and $y^{*}=\left(y_{1}^{*}, \ldots, y_{s}^{*}\right), x_{s}^{*} \neq y_{s}^{*}$;

(A3) the ideal $\mathcal{I}\left(\left\{\left.h_{1}\right|_{a=a^{*}},\left.h_{2}\right|_{a=a^{*}}, \ldots,\left.h_{s}\right|_{a=a^{*}}\right\}\right)$ is radical.

Remark 6.8. When (A1) holds, the assumption (A3) implies that each complex solution $x^{*}=$ $\left(x_{1}^{*}, x_{2}^{*}, \ldots, x_{s}^{*}\right)$ has multiplicity 1 [6, Page 150, Corollary 2.6].

Remark 6.9. It is computationally expensive to verify whether a given system is general zerodimensional. In practice, one can take a heuristic approach: pick random values of $a_{1}, a_{2}, \ldots, a_{n}$, solve approximately for the resulting complex solutions, and then check whether conditions (A1)(A3) in Definition 6.7 are satisfied. If this is the case, then we assume that the given system is general zero-dimensional and we try to look for the triangular form described in Theorem 6.11. 
Remark 6.10 (Relation to Shape Lemma). Theorem 6.11 can be viewed as a more general version of the Shape Lemma. The original Shape Lemma 2] pertains to a zero-dimensional ideal arising from a polynomial system without parameters. Later, a version for systems involving parameters was given by geometric resolutions [20, 21. The main difference between Theorem 6.11 and the results in [20, 21] is that in Theorem 6.11, a triangular system representing the solution set of a general zero-dimensional ideal is selected from a Gröbner basis, whereas in [20, 21, a triangular system is computed by an interpolation idea.

Theorem 6.11. Suppose that $h_{1}, h_{2}, \ldots, h_{s} \in \mathbb{C}[a, x]$ form a general zero-dimensional system. Let $\mathcal{G}$ be a Gröbner basis of the following ideal with respect to the lexicographic order $a_{n}<\cdots<a_{2}<$ $a_{1}<x_{s}<\cdots<x_{2}<x_{1}$ :

$$
\mathcal{I}\left(\left\{h_{1}, h_{2}, \ldots, h_{s}\right\}\right) \subseteq \mathbb{C}[a, x] .
$$

Then there exist $g_{1}, g_{2}, \ldots, g_{s} \in \mathcal{G}$ such that:

(1) $g_{1}, g_{2}, \ldots, g_{s}$ have the following triangular form:

$$
\begin{aligned}
g_{s} & =Q_{s, N} x_{s}^{N}+Q_{s, N-1} x_{s}^{N-1}+\ldots+Q_{s, 1} x_{s}+Q_{s, 0}, \\
g_{s-1} & =Q_{s-1} x_{s-1}+R_{s-1}, \\
& \vdots \\
g_{1} & =Q_{1} x_{1}+R_{1},
\end{aligned}
$$

where $N>0$ and, for all $i \in\{0,1, \ldots, N\}$ and $j \in\{1,2, \ldots, s-1\}$, we have:

$$
Q_{s, i} \in \mathbb{C}[a], \quad Q_{j} \in \mathbb{C}[a], \quad R_{j} \in \mathbb{C}\left[a, x_{j+1}, \ldots, x_{s}\right] .
$$

(2) For any $a^{*} \in \mathbb{C}^{n} \backslash V\left(Q_{s, N} Q_{1} Q_{2} \cdots Q_{s-1}\right)$, the set $\left\{\left.g_{1}\right|_{a=a^{*}},\left.g_{2}\right|_{a=a^{*}},\left.\ldots g_{s}\right|_{a=a^{*}}\right\}$ is a Gröbner basis of the following ideal with respect to the lexicographic order $x_{s}<\cdots<x_{2}<x_{1}$ :

$$
\mathcal{I}\left(\left\{\left.h_{1}\right|_{a=a^{*}},\left.h_{2}\right|_{a=a^{*}}, \ldots,\left.h_{s}\right|_{a=a^{*}}\right\}\right) \subseteq \mathbb{C}\left[x_{1}, x_{2}, \ldots, x_{s}\right] .
$$

The proof of Theorem 6.11 is in Appendix C.

Corollary 6.12. Let $G$ be a reaction network with s species and a simplified system $h_{c, a}(x)$. If $h_{c, a, 1}, h_{c, a, 2}, \ldots, h_{c, a, s}$ form a general zero-dimensional system, then the steady-state equations of $G$ admit a triangular form.

Proof. Straightforward from Definition 6.2. Theorem 6.11, and the fact that if $\left(c^{*}, a^{*}\right)$ is not in $W$, then the same is true for any sufficiently small perturbation of $\left(c^{*}, a^{*}\right)$.

We end this section by showing, through the Calvin cycle example, how to use Theorem 6.5 to find a witness to multistationarity.

Example 6.13 (Calvin cycle, continued). We return to the network in Example 6.1, which is known to be multistationarity [23]. Here we find a witness to multistationarity. 
Consider the following upper-triangular matrix:

$$
M(\kappa)=\left(\begin{array}{ccccccccccc}
1 & 1 & 0 & 0 & 0 & 0 & 0 & 0 & 0 & 0 & 0 \\
0 & \frac{1}{\kappa_{1}} & 0 & 0 & 0 & 0 & 0 & 0 & 0 & 0 & 0 \\
0 & 0 & 1 & 1 & 0 & 0 & 0 & 0 & 0 & 0 & 0 \\
0 & 0 & 0 & \frac{1}{\kappa_{3}} & 0 & 0 & 0 & 0 & 0 & 0 & 0 \\
0 & 0 & 0 & 0 & 1 & 1 & 0 & 0 & 0 & 0 & 0 \\
0 & 0 & 0 & 0 & 0 & \frac{1}{\kappa_{5}} & 0 & 0 & 0 & 0 & 0 \\
0 & 0 & 0 & 0 & 0 & 0 & 1 & 5 & 0 & 0 & 1 \\
0 & 0 & 0 & 0 & 0 & 0 & 0 & \frac{1}{\kappa_{7}} & 0 & 0 & 0 \\
0 & 0 & 0 & 0 & 0 & 0 & 0 & 0 & 1 & 1 & 0 \\
0 & 0 & 0 & 0 & 0 & 0 & 0 & 0 & 0 & \frac{1}{\kappa_{9}} & 0 \\
0 & 0 & 0 & 0 & 0 & 0 & 0 & 0 & 0 & 0 & \frac{1}{\kappa_{11}}
\end{array}\right) .
$$

Note that $\operatorname{det} M(\kappa)>0$ for $\kappa \in \mathbb{R}_{>0}^{10}$. Let the effective parameters be:

$$
\begin{aligned}
& \bar{a}_{1}=\kappa_{2}, \quad \bar{a}_{2}=\kappa_{4}, \quad \bar{a}_{3}=\kappa_{6}, \quad \bar{a}_{4}=\kappa_{8}, \quad \bar{a}_{5}=\kappa_{10}, \quad \bar{a}_{6}=\kappa_{12}, \\
& \bar{a}_{7}=\frac{\kappa_{2}}{\kappa_{1}}, \quad \bar{a}_{8}=\frac{\kappa_{4}}{\kappa_{3}}, \quad \bar{a}_{9}=\frac{\kappa_{6}}{\kappa_{5}}, \quad \bar{a}_{10}=\frac{\kappa_{8}}{\kappa_{7}}, \quad \bar{a}_{11}=\frac{\kappa_{10}}{\kappa_{9}}, \quad \bar{a}_{12}=\frac{\kappa_{12}}{\kappa_{11}} .
\end{aligned}
$$

From the above effective parameters (35) and equations (8)-(10), the resulting system $h_{c, a}(x)$ is:

$$
\begin{array}{ll}
h_{c, a, 1}=-a_{1} x_{3}+a_{5} x_{15}, & h_{c, a, 2}=x_{2}+x_{3}-c_{1} \\
h_{c, a, 3}=x_{1} x_{2}-a_{7} x_{3}, & h_{c, a, 4}=2 a_{1} x_{3}-a_{2} x_{6} \\
h_{c, a, 5}=x_{5}+x_{6}-c_{2}, & h_{c, a, 6}=x_{4} x_{5}-a_{8} x_{6} \\
h_{c, a, 7}=a_{2} x_{6}-a_{3} x_{9}, & h_{c, a, 8}=x_{8}+x_{9}-c_{3} \\
h_{c, a, 9}=x_{7} x_{8}-a_{9} x_{9}, & h_{c, a, 10}=a_{3} x_{9}-5 a_{4} x_{12}-a_{6} x_{17}, \\
h_{c, a, 11}=x_{11}+x_{12}-c_{4}, & h_{c, a, 12}=x_{10}^{5} x_{11}-a_{10} x_{12}, \\
h_{c, a, 13}=3 a_{4} x_{12}-a_{5} x_{15}, & h_{c, a, 14}=x_{14}+x_{15}-c_{5} \\
h_{c, a, 15}=x_{13} x_{14}-a_{11} x_{15}, & h_{c, a, 16}=x_{16}+x_{17}-c_{6}, \\
h_{c, a, 17}=x_{10} x_{16}-a_{12} x_{17} . &
\end{array}
$$

With an eye toward applying Theorem 6.5, we find a triangular form for our steady-state equations, as follows. Following the heuristic proposed in Remark 6.9, we verified for several values of the $a_{i}$ 's and $c_{i}$ 's that (A1), (A2), and (A3) in Definition 6.7 are satisfied. Hence, we assume that the $h_{c, a, i}$ 's form a general zero-dimensional system. If so, Corollary 6.12 would imply that the steady-state equations admit a triangular form. More precisely, we would obtain such a triangular set as a subset of a Gröbner basis (by Theorem 6.11).

Accordingly, we compute a Gröbner basis of the generated ideal $\left\langle h_{c, a, i}\right\rangle$ with respect to the lexicographic order $c<a<x_{17}<\ldots<x_{1}$, via Maple 34, which consists of 86 polynomials. Following the proof of Theorem 6.11 (see Appendix C), we select for each $i=1,2, \ldots, 17$, some $g_{i}$ among these 86 polynomials for which the leading monomial of $g_{i}$, where $g_{i}$ viewed in $\mathbb{Q}\left(a_{i}, c_{i}\right)[x]$, has the form $x_{i}^{N_{i}}$ for some $N_{i} \geq 0$. This yields the following 17 polynomials $g_{i}$, which form a 
triangular set:

$$
\begin{aligned}
g_{17}= & a_{6}\left(a_{10}-a_{12}^{5}\right) x_{17}^{6}+\left(a_{4} a_{12}^{5} c_{4}-5 a_{6} a_{10} c_{6}\right) x_{17}^{5}+10 a_{6} a_{10} c_{6}^{2} x_{17}^{4} \\
& -10 a_{6} a_{10} c_{6}^{3} x_{17}^{3}+5 a_{6} a_{10} c_{6}^{4} x_{17}^{2}-a_{6} a_{10} c_{6}^{5} x_{17}, \\
g_{16}= & x_{16}+x_{17}-c_{6} \\
g_{15}= & a_{5} x_{15}-3 a_{6} x_{17} \\
g_{14}= & x_{14}+x_{15}-c_{5} \\
\vdots & \\
g_{3}= & a_{1} x_{3}-3 a_{6} x_{17} \\
g_{2}= & x_{2}+x_{3}-c_{1} \\
g_{1}= & Q_{1}(a, c) x_{1}+R_{1}\left(a, c, x_{17}\right),
\end{aligned}
$$

where $Q_{1}(a, c)$ and $R_{1}\left(a, c, x_{17}\right)$ are polynomials. So, by Theorem 6.11 (2), the steady-state equations admit the following triangular form:

$$
\begin{gathered}
T_{17}=g_{17}\left(a, c, x_{17}\right), T_{16}=x_{16}-\left(c_{6}-x_{17}\right), T_{15}=x_{15}-3 \frac{a_{6}}{a_{5}} x_{17}, T_{14}=x_{14}-\left(c_{5}-x_{15}\right) \\
\cdots \\
T_{3}=x_{3}-3 \frac{a_{6}}{a_{1}} x_{17}, T_{2}=x_{2}-\left(c_{1}-x_{3}\right), T_{1}=x_{1}-\frac{R_{1}\left(a, c, x_{17}\right)}{Q_{1}(a, c)} .
\end{gathered}
$$

This triangular form is valid as long as the leading coefficients of $g_{i}$ 's do not vanish. In other words, the variety $\mathcal{W}$, as in Definition 6.2 can be defined by the vanishing set of those coefficients.

Next, we aim to find a degenerate positive steady state $x^{*}$ and corresponding parameters $\left(a^{*}, c^{*}\right)$. Let $\hat{a}=a_{6}$ and $\hat{x}=x$. The idea is to compute the critical function $C(\hat{a}, \hat{x})$ and then find a positive point where the function vanishes. By solving the equations $h_{c, a, 1}=h_{c, a, 3}=h_{c, a, 4}=h_{c, a, 6}=$ $h_{c, a, 7}=h_{c, a, 8}=h_{c, a, 9}=h_{c, a, 10}=h_{c, a, 12}=h_{c, a, 13}=h_{c, a, 15}=h_{c, a, 17}=0$ in the unknowns $a_{1}, a_{2}, a_{3}, a_{4}, a_{5}, a_{7}, a_{8}, a_{9}, a_{10}, a_{11}, a_{12}$, we obtain:

$$
\begin{aligned}
& a_{1}=\frac{3 a_{6} x_{17}}{x_{3}}, \quad a_{2}=\frac{6 a_{6} x_{17}}{x_{6}}, \quad a_{3}=\frac{6 a_{6} x_{17}}{x_{9}}, \quad a_{4}=\frac{a_{6} x_{17}}{x_{12}}, \quad a_{5}=\frac{3 a_{6} x_{17}}{x_{15}}, \\
& a_{7}=\frac{x_{1} x_{2}}{x_{3}}, \quad a_{8}=\frac{x_{4} x_{5}}{x_{6}}, \quad a_{9}=\frac{x_{7} x_{8}}{x_{9}}, \quad a_{10}=\frac{x_{10}^{5} x_{11}}{x_{12}}, \quad a_{11}=\frac{x_{13} x_{14}}{x_{15}}, \quad a_{12}=\frac{x_{10} x_{16}}{x_{17}} .
\end{aligned}
$$

Thus, we obtain the following steady-state parametrization (outside $\mathcal{W}$ ) $\phi: \mathbb{R}_{>0}^{18} \rightarrow \mathbb{R}_{>0}^{12} \times \mathbb{R}_{>0}^{17}$, where $\phi(\hat{a}, \hat{x})=\phi\left(a_{6} ; x\right)$ is defined as

$$
\left(\frac{3 a_{6} x_{17}}{x_{3}}, \frac{6 a_{6} x_{17}}{x_{6}}, \frac{6 a_{6} x_{17}}{x_{9}}, \frac{a_{6} x_{17}}{x_{12}}, \frac{3 a_{6} x_{17}}{x_{15}}, a_{6}, \frac{x_{1} x_{2}}{x_{3}}, \frac{x_{4} x_{5}}{x_{6}}, \frac{x_{7} x_{8}}{x_{9}}, \frac{x_{10}^{5} x_{11}}{x_{12}}, \frac{x_{13} x_{14}}{x_{15}}, \frac{x_{10} x_{16}}{x_{17}} ; x\right) .
$$

The resulting critical function is:

$$
C(\hat{a}, \hat{x})=C\left(a_{6}, x\right)=-324 a_{6}^{5} x_{2} x_{5} x_{8} x_{10}^{5} x_{14} x_{17}^{4}\left(4 x_{11} x_{16}+5 x_{11} x_{17}-x_{12} x_{16}\right) .
$$

It is straightforward to find a positive rational vector $\left(a_{6}^{*}, x^{*}\right)$ such that $C\left(a_{6}^{*}, x^{*}\right)$ vanishes. For instance, one can simply choose

$$
a_{6}^{*}=1 \text { and } x^{*}=(1,1,1,1,1,1,1,1,1,1,1,9,1,1,1,1,1) .
$$


Plugging $u^{*}$ into $\phi(u)$, we compute:

$$
a^{*}=\left(3,6,6, \frac{1}{9}, 3,1,1,1,1, \frac{1}{9}, 1,1\right) .
$$

Also, by plugging $x^{*}$ into the conservation laws, we obtain:

$$
c^{*}=(2,2,2,10,2,2) \text {. }
$$

Then $x^{*}$ is a degenerate positive steady state for $\left(a^{*}, c^{*}\right)$. In other words, we have verified the first hypothesis of Theorem 6.5. It is straightforward to check the validity of the remaining three hypotheses of this theorem. Thus, by Theorem 6.5, there exists a small positive number $\delta$ such that for any $0<\delta^{\prime}<\delta$

$$
\left(a_{1}^{*}, \ldots, a_{3}^{*}, a_{4}^{*}+\delta^{\prime}, a_{5}^{*}, \ldots, a_{12}^{*}, c^{*}\right) \quad \text { or } \quad\left(a_{1}^{*}, \ldots, a_{3}^{*}, a_{4}^{*}-\delta^{\prime}, a_{5}^{*}, \ldots, a_{12}^{*}, c^{*}\right)
$$

generates multistationarity. Indeed, one can check that the system

$$
h_{c, a}\left(a^{* *}, c^{*}, x\right)=0, \quad \text { where } a^{* *}=\left(a_{1}^{*}, \ldots, a_{3}^{*}, a_{4}^{*}-\frac{1}{1000}, a_{5}^{*}, \ldots, a_{12}^{*}\right),
$$

has two distinct positive steady states, which are approximately equal to:

$x^{(1)} \approx(0.96,1.01,0.98,0.96,1.01,0.98,0.96,1.01,0.98,0.96,1.14,8.85,0.96,1.01,0.98,1.01,0.98)$, and

$x^{(2)} \approx(1.02,0.98,1.01,1.02,0.98,1.01,1.02,0.98,1.01,1.02,0.87,9.12,1.02,0.98,1.01,0.98,1.01)$.

Finally, any $\kappa^{*} \in \mathbb{R}_{>0}^{12}$ for which $\bar{a}\left(\kappa^{*}\right)=a^{* *}$, as in (35), yields a witness to multistationarity. One such $\kappa^{*}$ is:

$$
\kappa^{*}=\left(3,3, \frac{5999}{1000}, \frac{5999}{1000}, 6,6,1, \frac{1}{9}, 3,3,1,1\right) .
$$

\section{Discussion}

Steady-state parametrizations have been shown in recent years to be very useful for analyzing chemical reaction networks and more specifically their capacity for multistationarity. Our first main results are in this vein, discerning multistationarity and finding witnesses to multistationarity for networks with steady-state parametrizations (Theorem 3.12), including linearly binomial networks (Theorem 4.6). Furthermore, we characterize a class of MESSI networks that are linearly binomial, which provides an interesting range of application for our results. In a complementary direction, we also showed how to obtain witnesses to multistationarity when a network's steady-state equations admit a triangular form and a degenerate steady state exists (Theorem 6.5).

Hence, the main contribution of our work is a suite of new tools for tackling the important but difficult problem of assessing and analyzing multistationarity. Moreover, our results can decide multistationarity for networks from biology that previously could not be handled systematically.

Finally, our work used novel approaches that we expect to be useful in the future. For instance, we used steady-state parametrizations in which the rate constants depend on the species variables, thereby simplifying the subsequent analyses. We also analyzed reaction networks based on results on specialization of Gröbner bases, adapting a general technique used in several applications. We expect similar algebraic techniques to allow us in the future to go beyond multistationarity to study topics such as stability of steady states and Hopf bifurcations. 
Acknowledgements. The authors thank Frank Sottile for helpful discussions, Alan Rendall for pointing us to the Calvin Cycle model, and Carsten Conradi for helpful discussions on the ERK network. The authors also thank three conscientious referees whose comments helped improve our work. AD and MPM were partially supported by UBACYT 20020170100048BA, CONICET PIP 11220150100473 and 11220150100483, and ANPCyT PICT 2016-0398, Argentina. AS partially supported by the NSF (DMS-1513364 and DMS-1752672) and the Simons Foundation (\#521874). XT was partially supported by the NSF (DMS-1752672).

\section{REFERENCES}

[1] Murad Banaji and Casian Pantea. Some results on injectivity and multistationarity in chemical reaction networks. SIAM J. Appl. Dyn. Syst., 15(2):807-869, 2016.

[2] Eberhard Becker, Maria Grazia Marinari, Teo Mora, and Carlo Traverso. The shape of the Shape Lemma. In Proceedings of ISSAC '94, pages 129-133. ACM New York, 1994.

[3] Frederic Bihan, Alicia Dickenstein, and Magali Giaroli. Lower bounds for positive roots and regions of multistationarity in chemical reaction networks. Preprint, arXiv:1807.05157.

[4] Carsten Conradi, Elisenda Feliu, Maya Mincheva, and Carsten Wiuf. Identifying parameter regions for multistationarity. PLoS Comput. Biol., 13(10):e1005751, 2017.

[5] Carsten Conradi and Anne Shiu. Dynamics of post-translational modification systems: Recent progress and future challenges. Biophys. J., 114(3):507-515, 2018.

[6] David Cox, Jon Little, and Donal O'Shea. Using Algebraic Geometry, volume 185. Springer Science \& Business Media, 2005.

[7] David Cox, Jon Little, and Donal O'Shea. Ideals, varieties, and algorithms: An introduction to computational algebraic geometry and commutative algebra. Springer-Verlag, 2007.

[8] Gheorghe Craciun and Martin Feinberg. Multiple equilibria in complex chemical reaction networks. I. The injectivity property. SIAM J. Appl. Math., 65(5):1526-1546, 2005.

[9] Gheorghe Craciun and Martin Feinberg. Multiple equilibria in complex chemical reaction networks: Semiopen mass action systems. SIAM J. Appl. Math., 70(6):1859-1877, 2010.

[10] Gheorghe Craciun, J. William Helton, and Ruth J. Williams. Homotopy methods for counting reaction network equilibria. Math. Biosci., 216(2):140-149, 2008.

[11] Alicia Dickenstein. Biochemical reaction networks: An invitation for algebraic geometers. In Mathematical Congress of the Americas, volume 656, pages 65-83. American Mathematical Soc., 2016.

[12] German Enciso. Fixed points and convergence in monotone systems under positive or negative feedback. Int. J. Control, 87(2):301-311, 2014.

[13] Elisenda Feliu. Injectivity, multiple zeros and multistationarity in reaction networks. Proc. R. Soc. A, 471(2173), 2014.

[14] Elisenda Feliu and Carsten Wiuf. Enzyme-sharing as a cause of multi-stationarity in signalling systems. J. $R$. Soc. Interface, 9(71):1224-1232, 2012.

[15] Elisenda Feliu and Carsten Wiuf. Simplifying biochemical models with intermediate species. J. R. Soc. Interface, 10:20130484, 2013.

[16] Elisenda Feliu and Carsten Wiuf. Variable elimination in post-translational modification reaction networks with mass-action kinetics. J. Math. Biol., 66(1-2):281-310, 2013.

[17] Bryan Félix, Anne Shiu, and Zev Woodstock. Analyzing multistationarity in chemical reaction networks using the determinant optimization method. Appl. Math. Comput., 287-288:60-73, 2016.

[18] Israel Gelfand, Mikhail Kapranov, and Andrei Zelevinsky. Discriminants, resultants and multidimensional determinants. Birkhäuser Boston, 1994.

[19] Magali Giaroli, Frederic Bihan, and Alicia Dickenstein. Regions of multistationarity in cascades of GoldbeterKoshland loops. Preprint, arXiv: 1807.08400.

[20] Marc Giusti, Joos Heintz, Jose Enrique Morais, Jacques Morgenstern, and Luis Miguel Pardo. Straight-line programs in geometric elimination theory. Journal of Pure and Applied Algebra, 124(1):101-146, 1998. 
[21] Marc Giusti, Grégoire Lecerf, and Bruno Salvy. A Gröbner free alternative for polynomial system solving. J. Complexity, 17:154-211, 2001.

[22] Gilles Gnacadja. Reachability, persistence, and constructive chemical reaction networks (part iii): a mathematical formalism for binary enzymatic networks and application to persistence. J. Math. Chem., 49(10):2158-2176, 2011.

[23] Sergio Grimbs, Anne Arnold, Aneta Koseska, Jürgen Kurths, Joachim Selbig, and Zoran Nikoloski. Spatiotemporal dynamics of the Calvin cycle: Multistationarity and symmetry breaking instabilities. BioSystems, 103:212223, 2011.

[24] Katharina Holstein, Dietrich Flockerzi, and Carsten Conradi. Multistationarity in sequential distributed multisite phosphorylation networks. Bull. Math. Biol., 75(11):2028-2058, 2013.

[25] Matthew D. Johnston. Translated chemical reaction networks. Bull. Math. Biol., 76(6):1081-1116, 2014.

[26] Badal Joshi. Complete characterization by multistationarity of fully open networks with one non-flow reaction. Appl. Math. Comput., 219:6931-6945, 2013.

[27] Badal Joshi and Anne Shiu. A survey of methods for deciding whether a reaction network is multistationary. Math. Model. Nat. Phenom., special issue on "Chemical dynamics", 10(5):47-67, 2015.

[28] Badal Joshi and Anne Shiu. Which small reaction networks are multistationary? SIAM J. Appl. Dyn. Syst., 16(2):802-833, 2017.

[29] Deepak Kapur, Yao Sun, and Dingkang Wang. A new algorithm for computing comprehensive Gröbner systems. In ISSAC'10 Proceedings of the 35th International Symposium on Symbolic and Algebraic Computation, pages 29-36, 2010.

[30] Matthew Johnston, Stefan Müller, and Casian Pantea. A deficiency-based approach to parametrizing positive equilibria of biochemical reaction systems. Preprint, arXiv:1805.09295.

[31] Inom Mirzaev and Jeremy Gunawardena. Laplacian dynamics on general graphs. Bull. Math. Biol., 75(11):211849, 2013.

[32] Stefan Müller, Elisenda Feliu, Georg Regensburger, Carsten Conradi, Anne Shiu, and Alicia Dickenstein. Sign conditions for injectivity of generalized polynomial maps with applications to chemical reaction networks and real algebraic geometry. Found. Comput. Math., 16(1):69-97, 2016.

[33] Stefan Müller, Josef Hofbauer, and Georg Regensburger. On the bijectivity of families of exponential/generalized polynomial maps. Preprint, arXiv:1804.01851.

[34] Maple 17 (2013) Maplesoft, a division of Waterloo Maple Inc., Waterloo, Ontario.

[35] Mercedes Pérez Millán and Alicia Dickenstein. The structure of MESSI biological systems. SIAM J. Appl. Dyn. Syst., 17(2):1650-1682, 2018.

[36] Mercedes Pérez Millán, Alicia Dickenstein, Anne Shiu, and Carsten Conradi. Chemical reaction systems with toric steady states. Bull. Math. Biol., 74(5):1027-1065, 2012.

[37] Boris Y. Rubinstein, Henry H. Mattingly, Alexander M. Berezhkovskii, and Stanislav Y. Shvartsman. Long-term dynamics of multisite phosphorylation. Mol. Biol. Cell, 27(14):2331-2340, 2016.

[38] AmirHosein Sadeghimanesh and Elisenda Feliu. The multistationarity structure of networks with intermediates and a binomial core network. Preprint, arXiv: 1808.07548.

[39] Guy Shinar and Martin Feinberg. Concordant chemical reaction networks. Math. Biosci., 240(2):92-113, 2012.

[40] Anne Shiu. The smallest multistationary mass-preserving chemical reaction network. Lect. Notes Comput. Sc., 5147:172-184, 2008.

[41] Anne Shiu and Timo de Wolff. Nondegenerate multistationarity in small reaction networks. Preprint, arXiv: 1802.00306, 2018.

[42] Anne Shiu and Bernd Sturmfels. Siphons in chemical reaction networks. Bull. Math. Biol., 72(6):1448-1463, 2010.

[43] Matthew Thomson and Jeremy Gunawardena. The rational parameterisation theorem for multisite posttranslational modification systems. J. Theoret. Biol., 261(4):626-636, 2009.

[44] William T. Tutte. The dissection of equilateral triangles into equilateral triangles. Math. Proc. Cambridge, 44(4):463-482, 1948.

[45] Liming Wang and Eduardo D. Sontag. On the number of steady states in a multiple futile cycle. J. Math. Biol., 57(1):29-52, 2008.

[46] Carsten Wiuf and Elisenda Feliu. Power-law kinetics and determinant criteria for the preclusion of multistationarity in networks of interacting species. SIAM J. Appl. Dyn. Syst., 12:1685-1721, 2013. 


\section{Appendix A. Proof of Theorem 5.3}

We now prove Theorem 5.3. We also illustrate the proof in Example A.1. We assume the reader is familiar with the notion of the Laplacian $\mathcal{L}(G)$ of a digraph $G$ and its main properties. One important observation is that mass-action kinetics associated with a digraph $G$ with vertices labeled by variables $x_{1}, \ldots, x_{s}$ equals $\dot{x}=\mathcal{L}(G) x$. Another important observation is that when $G$ is strongly connected, the kernel of $\mathcal{L}(G)$ has dimension one and there is a known generator $\rho(G)$ with positive entries described as follows. Recall that an $i$-tree $T$ of a digraph is a spanning tree where the $i$-th vertex is its unique sink (equivalently, the $i$-th is the only vertex of the tree with no edges leaving from it) and we call $k^{T}$ the product of the labels of all the edges of $T$. Then, the $i$-th coordinate of $\rho(G)$ equals

$$
\rho_{i}(G)=\sum_{\text {Tan } i-\text { tree }} k^{T}
$$

We refer the reader to [31, 44] for a detailed account.

Proof of Theorem 5.3. Recall that in a MESSI network there are two types of species: intermediate and core. Our proof proceeds by performing (invertible) linear operations on the steady-state equations, which in the end yield (equivalent) binomial equations.

We begin by operating on the steady-state equations of intermediate species. Given a core complex $y$, we consider the following set of intermediate complexes: $I_{y}=\left\{y^{\prime}\right.$ intermediate $: y \rightarrow \circ$ $\left.y^{\prime}\right\}$. Following the reasoning in [15], we build a labeled directed graph denoted by $G_{y}$, with node set $I_{y} \cup\{y\}$, and labeled directed edges as in $G$, except that any reaction of the form $y^{\prime} \stackrel{\kappa}{\rightarrow} y^{\prime \prime}$, where $y^{\prime} \in I_{y}$ and $y^{\prime \prime}$ is any core complex, is replaced by $y^{\prime} \stackrel{\kappa}{\rightarrow} y$, with the same rate constant (if there are several core complexes to which $y^{\prime}$ reacts, the edges are collapsed and the label equals the sum of the labels of the corresponding collapsed edges). Note that, as all intermediate complexes in MESSI networks react via intermediates to some core complex, the graph $G_{y}$ is strongly connected.

Number the species in $I_{y}$ and denote by $x_{1}, \ldots, x_{n_{y}}$ the corresponding concentrations. Then, the mass-action ODEs corresponding to them in the given network, are given by $f_{\ell}=\dot{x}_{\ell}=\left(\mathcal{L}\left(G_{y}\right)\right)_{\ell} \mathbf{x}^{\top}$, where $\left(\mathcal{L}\left(G_{y}\right)\right)_{\ell}$ is the $\ell$ th row of the Laplacian $\mathcal{L}\left(G_{y}\right)$ of $G_{y}, \mathbf{x}=\left(x_{1}, \ldots, x_{n_{y}}, m(y)\right)$, and $m(y)$ is the monomial associated with the complex $y$. Call $\rho_{\ell}=\rho\left(G_{y}\right)_{\ell}$ for $1 \leq \ell \leq n_{y}+1$. From the Matrix-Tree Theorem we know that, up to sign, the determinant of the first $n_{y} \times n_{y}$ principal minor of $\mathcal{L}\left(G_{y}\right)$ equals $\rho_{n_{y}+1} \neq 0$. Call $A$ the $\left(n_{y}+1\right) \times\left(n_{y}+1\right)$ block matrix

$$
A=\left(\begin{array}{c|c}
A_{1} & 0 \\
\hline 0 & 1
\end{array}\right)
$$

where $A_{1}$ is the $n_{y} \times n_{y}$ is such that $A \cdot \mathcal{L}\left(G_{y}\right)$ has the form:

$$
A \cdot \mathcal{L}\left(G_{y}\right)=\left(\begin{array}{ccc|c}
1 & & 0 & -\alpha_{1} \\
& \ddots & & \vdots \\
0 & & 1 & -\alpha_{n_{y}} \\
\hline * & \ldots & * & *
\end{array}\right) .
$$

Such a matrix $A$ exists since the $n_{y} \times n_{y}$ first principal minor of $\mathcal{L}\left(G_{y}\right)$ is invertible. Observe that $\operatorname{ker}\left(\mathcal{L}\left(G_{y}\right)\right)$ is generated by $\left(\rho_{1}, \rho_{2}, \ldots, \rho_{n_{y}}, \rho_{n_{y}+1}\right)$, so $\rho_{\ell}-\alpha_{\ell} \rho_{n_{y}+1}=0$, for $1 \leq \ell \leq n_{y}$, and then $\alpha_{\ell}=\frac{\rho_{\ell}}{\rho_{n_{y}+1}}\left(\right.$ and $\left.\alpha_{\ell} \neq 0\right)$. By multiplying $\mathcal{L}\left(G_{y}\right)$ on the left by $A$, which is equivalent to operating 
linearly on the original equations $f_{\ell}=0$, we deduce the binomial equations $x_{\ell}-\alpha_{\ell} m(y)=0$, for the intermediate species $x_{\ell}$ at steady state, for $1 \leq \ell \leq n_{y}$. Note that, as $\rho_{n_{y}+1} \neq 0$ for all $\kappa \in \mathbb{R}_{>0}^{m}$, these operations are well defined for all $\kappa \in \mathbb{R}_{>0}^{m}$.

We can afterwards substitute the steady-state value of the intermediate species $x_{\ell} \in I_{y}$ into the original steady-state equations, for core species, of $G$. We moreover show that this substitution can be achieved via linear operations. Indeed, for a core species $X_{k}$, write the corresponding ODE as:

$$
f_{k}=\dot{x}_{k}=p_{k}+\sum_{\ell=1}^{n_{y}} \kappa_{\ell} x_{\ell},
$$

where $p_{k}$ is a polynomial that does not depend on $x_{1}, \ldots, x_{n_{y}}$ and $\kappa_{\ell} \geq 0$ is positive precisely when $X_{\ell}$ reacts with rate constant $\kappa_{\ell}$ to a core complex that involves $X_{k}$. We now subtract a linear combination of the intermediate-species binomials $\left(x_{\ell}-\alpha_{\ell} m(y)\right)$ :

$$
f_{k}-\sum_{\ell=1}^{n_{y}} \kappa_{\ell}\left(x_{\ell}-\alpha_{\ell} m(y)\right)=p_{k}+\sum_{\ell=1}^{n_{y}}\left(\kappa_{\ell} \alpha_{\ell}\right) m(y),
$$

and so we replace $f_{k}=0$ by $p_{k}+\sum_{\ell=1}^{n_{y}}\left(\kappa_{\ell} \alpha_{\ell}\right) m(y)=0$, where all the intermediates in $I_{y}$ have been eliminated by performing linear operations on the original steady-state equations (for core species) and the new binomials (for the intermediates).

A key observation pertaining to how we obtained binomial equations for all intermediate species is that, by condition $(\mathcal{C})$, the set of intermediate complexes can be written as the disjoint union of sets $I_{y}$ for a certain (finite) number of core complexes $y$. By the natural bijection between intermediate complexes and intermediate species, we can then obtain the corresponding binomials by operating linearly on the original equations. We also, as described above, eliminated all the intermediate species from the core-species equations by linear operations. We will denote this procedure as follows. First assume, without loss of generality, that the intermediate species are the last $s-n$ species. Then we can assert that there exists an invertible matrix $M_{1}=M_{1}(\kappa) \in \mathbb{Q}(\kappa)^{s \times s}$ which is well defined for all $\kappa \in \mathbb{R}_{>0}^{m}$ such that $M_{1}\left(f_{1}, \ldots, f_{s}\right)^{\top}=\left(\tilde{f}_{1}, \ldots, \tilde{f}_{n}, \tilde{h}_{n+1}, \ldots, \tilde{h}_{s}\right)^{\top}$, where $\tilde{f}_{1}, \ldots, \tilde{f}_{n}$ do not depend on the intermediate-species concentrations $x_{n+1}, \ldots, x_{s}$, and $\tilde{h}_{n+\ell}$ is the binomial for the intermediate species $x_{n+\ell}(1 \leq \ell \leq s-n)$ and its form is $x_{n+\ell}-\alpha_{n+\ell} m(y)=0$.

Before we continue operating on the core-species equations (the $\tilde{f}_{i}$ 's are not binomials), we describe the map $\boldsymbol{\tau}=\boldsymbol{\tau}(\kappa)$ mentioned in (34). For each $X_{i}+X_{j} \rightarrow_{\circ} X_{\ell}+X_{m}$ in $G$, the reaction constant $\tau$ in $G_{1}$ which gives the label $X_{i}+X_{j} \stackrel{\tau}{\longrightarrow} X_{\ell}+X_{m}$ has the form

$$
\tau=\kappa+\sum_{k=1}^{s-n} \kappa_{k} \alpha_{k}
$$

where $\kappa \geq 0$ is positive when $X_{i}+X_{j} \stackrel{\kappa}{\longrightarrow} X_{\ell}+X_{m}$ in $G$ (and $\kappa=0$ otherwise), and $\kappa_{k} \geq 0$ is positive if there is a reaction from the intermediate species $X_{n+k} \stackrel{\kappa_{k}}{\longrightarrow} X_{\ell}+X_{m}$ and $X_{i}+X_{j} \rightarrow_{\circ} X_{n+k}$ in $G$ (and $\kappa_{k}=0$ otherwise). As we pointed out in Remark 5.2, the steady states of $G$ are in one-to-one correspondence with those of $G_{1}$ and, in fact, the polynomials $\tilde{f}_{i}$ can be read from the digraph $G_{1}$ (see Theorem 3.2 in [15]).

What we show now is that we can operate linearly on the core-species equations $\tilde{f}_{i}=0$ (for $1 \leq i \leq n)$ in order to obtain equivalent binomial equations. To avoid unnecessary notation, we 
will assume in what follows that the partition of $\mathscr{S}$ is minimal. Recall that a vertex in a directed graph has outdegree zero if it is not the tail of any directed edge. Let us define subsets of indices based on the graph $G_{E}$ :

$$
\begin{aligned}
& L_{0}=\left\{\beta \geq 1: \text { outdegree of } \mathscr{S}^{(\beta)} \text { is } 0\right\} \\
& L_{k}=\left\{\beta \geq 1 \text { : for any edge } \mathscr{S}^{(\beta)} \rightarrow \mathscr{S}^{(\gamma)} \text { in } G_{E} \text { it holds that } \gamma \in L_{t}, \text { with } t<k\right\} \backslash \bigcup_{t=0}^{k-1} L_{t}, k \geq 1 .
\end{aligned}
$$

As $\mathscr{S}$ is finite and there are no directed cycles in $G_{E}$, there must exist a subset $\mathscr{S}^{(\beta)}$ with $1 \leq \beta \leq m$ such that its outdegree in $G_{E}$ is zero. This means that $L_{0} \neq \emptyset$.

Consider $\alpha \in L_{0}$. By the assumption of minimality of the partition, there is a connected component of $G_{2}^{\circ}$, which we denote by $H_{\alpha}$, with vertices the species in $\mathscr{S}^{(\alpha)}$. Let $\widetilde{H}_{\alpha}$ be the corresponding underlying undirected graph. As $\widetilde{H}_{\alpha}$ is a tree, consider $X_{i}$, a leaf of the tree (this is, a vertex of degree one). $X_{i}$ is only connected to one vertex $X_{j}$, so $\tilde{f}_{i}$ is already a binomial of the form

$$
\tilde{f}_{i}=\tau_{j i} x_{j} x_{\ell}-\tau_{i j} x_{i} x_{h}
$$

for some species $X_{\ell} \in \mathscr{S}^{(\beta)}, X_{h} \in \mathscr{S}^{(\gamma)}, \beta, \gamma$ in levels strictly greater than 0. Moreover, $\tilde{f}_{j}$ is

$$
\tilde{f}_{j}=p_{j}+\tau_{i j} x_{i} x_{h}-\tau_{j i} x_{j} x_{\ell}=p_{j}-\tilde{f}_{i},
$$

with $p_{j}$ a polynomial that does not depend on $x_{i}$. Then, $\tilde{f}_{j}+\tilde{f}_{i}=p_{j}$. And we can replace $\tilde{f}_{j}$ with $p_{j}$. As the associated digraph $G_{E}$ has no directed cycles, all the reactions are enzymatic. This means that the reactions that correspond to $\tau_{i j}$ and $\tau_{j i}$ in $G_{1}$ are $X_{i}+X_{h} \stackrel{\tau_{i j}}{\longrightarrow} X_{j}+X_{h}$ and $X_{j}+X_{\ell} \stackrel{\tau_{j i}}{\longrightarrow} X_{i}+X_{\ell}$, respectively, and none of these reactions affect either $\tilde{f}_{h}$ or $\tilde{f}_{\ell}$. Moreover, as $X_{i} \in \mathscr{S}^{(\alpha)}$ with $\alpha \in L_{0}$ and $\mathscr{S}^{(\alpha)}$ has outdegree zero in $G_{E}, x_{i}$ only appears in $\tilde{f}_{i}$ and $\tilde{f}_{j}$. We have then eliminated by linear operations the variable $x_{i}$ from all the equations other than $\tilde{f}_{i}$. And this can be done for all the species whose vertices are leaves of $\widetilde{H}_{\alpha}$. We can then erase all the leaves from $\widetilde{H}_{\alpha}$ and, by an inductive argument we see that we can operate linearly, with integer coefficients, on $\tilde{f}_{1}, \ldots, \tilde{f}_{n}$ to obtain binomials for the species in $\mathscr{S}^{(\alpha)}$. This argument holds for any $\alpha \in L_{0}$. As the species in the $\mathscr{S}^{(\alpha)}$ 's for $\alpha \in L_{0}$ do not appear in any label of the $L_{i}$ 's for $i \geq 1$ and all the reactions in all the $H_{\alpha}$ 's with $\alpha \in L_{0}$ do not affect the equations of those species that appear on its labels, by an inductive argument we can complete the proof to obtain binomial equations by operating linearly on the equations $\tilde{f}_{j}=0$ for the species in $\mathscr{S}^{(\alpha)}$ for $\alpha \in L_{i}, i \geq 1$.

We have then proved that there exists a matrix with integer entries $\widetilde{M}_{2} \in \mathbb{Q}^{n \times n}$, and an invertible block matrix $M_{2} \in \mathbb{Q}^{s \times s}$ of the form

$$
M_{2}=\left(\begin{array}{c|c}
\widetilde{M}_{2} & 0 \\
\hline 0 & I d_{s-n}
\end{array}\right),
$$

such that $M_{2}\left(\tilde{f}_{1}, \ldots, \tilde{f}_{n}, \tilde{h}_{n+1}, \ldots, \tilde{h}_{s}\right)^{\top}=\left(\tilde{h}_{1}, \ldots, \tilde{h}_{s}\right)^{\top}$, where $\tilde{h}_{1}, \ldots, \tilde{h}_{s}$ are binomials.

We have so far that there are invertible matrices $M_{1} \in \mathbb{Q}(\kappa)^{s \times s}$ and $M_{2} \in \mathbb{Q}^{s \times s}$, with $M_{1}$ well defined for all $\kappa \in \mathbb{R}_{>0}^{m}$, such that $M_{2} M_{1}\left(f_{1}, \ldots, f_{s}\right)^{\top}=\left(\tilde{h}_{1}, \ldots, \tilde{h}_{s}\right)^{\top}$, with $\operatorname{det}\left(M_{2} M_{1}\right) \neq 0$. If $f_{j_{1}}, \ldots, f_{j_{s-d}}$ is a basis of the $\mathbb{Q}(\kappa)$-linear subspace generated by $f_{1}, \ldots, f_{s}$ there must exist a set of $s-d$ binomials $\left\{\bar{h}_{j_{1}}, \ldots, \bar{h}_{j_{s-d}}\right\} \subseteq\left\{\tilde{h}_{1}, \ldots, \tilde{h}_{s}\right\}$ and an invertible matrix $M \in \mathbb{Q}(\kappa)^{(s-d) \times(s-d)}$, which 
is well defined for all $\kappa \in \mathbb{R}_{>0}^{m}$, such that $M\left(f_{j_{1}}, \ldots, f_{j_{s-d}}\right)^{\top}=\left(\bar{h}_{j_{1}}, \ldots, \bar{h}_{j_{s-d}}\right)^{\top}$, as we wanted to prove.

Example A.1. Consider the following network:

$$
\begin{gathered}
\mathrm{S}_{0}+\mathrm{E} \underset{\kappa_{2}}{\stackrel{\kappa_{1}}{\rightleftharpoons}} \mathrm{ES}_{0} \stackrel{\kappa_{3}}{\longrightarrow} \mathrm{S}_{1}+\mathrm{E} \stackrel{\kappa_{4}}{\longrightarrow} \mathrm{S}_{2}+\mathrm{E} \stackrel{\kappa_{5}}{\longrightarrow} \mathrm{S}_{3}+\mathrm{E} \\
\mathrm{S}_{3} \stackrel{\kappa_{6}}{\longrightarrow} \mathrm{S}_{2} \stackrel{\kappa_{7}}{\longrightarrow} \mathrm{S}_{1} \stackrel{\kappa_{8}}{\longrightarrow} \mathrm{S}_{0},
\end{gathered}
$$

which has $s=6$ species:

$$
X_{1}=\mathrm{S}_{0}, \quad X_{2}=\mathrm{S}_{1}, \quad X_{3}=\mathrm{S}_{2}, \quad X_{4}=\mathrm{S}_{3}, \quad X_{5}=\mathrm{E}, \quad X_{6}=\mathrm{ES}_{0} .
$$

There are 2 conservation laws:

$$
\begin{aligned}
x_{1}+x_{2}+x_{3}+x_{4}+x_{6} & =c_{1} \\
x_{5}+x_{6} & =c_{2} .
\end{aligned}
$$

The equations $f_{c, \kappa}(x)$ are

$$
\begin{array}{ll}
f_{c, \kappa, 1}=x_{1}+x_{2}+x_{3}+x_{4}+x_{6}-c_{1}, & f_{c, \kappa, 2}=\kappa_{3} x_{6}-\kappa_{4} x_{2} x_{5}+\kappa_{7} x_{3}-\kappa_{8} x_{2}, \\
f_{c, \kappa, 3}=\kappa_{4} x_{2} x_{5}-\kappa_{5} x_{3} x_{5}+\kappa_{6} x_{4}-\kappa_{7} x_{3}, & f_{c, \kappa, 4}=\kappa_{5} x_{3} x_{5}-\kappa_{6} x_{4}, \\
f_{c, \kappa, 5}=x_{5}+x_{6}-c_{2}, & f_{c, \kappa, 6}=\kappa_{1} x_{1} x_{5}-\left(\kappa_{2}+\kappa_{3}\right) x_{6} .
\end{array}
$$

The matrix $M_{1}(\kappa)$ is the product of two matrices: the first one multiplied by $\left(f_{1}, \ldots, f_{6}\right)^{\top}$ gives binomials for the intermediate species equations; the second one eliminates the intermediate species from the core species equations.

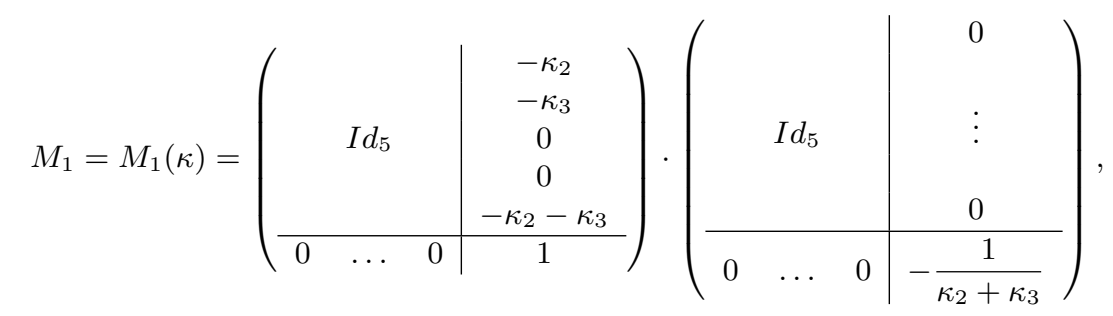

where $I d_{5}$ is the $5 \times 5$ identity matrix. This leads to:

$$
\begin{aligned}
M_{1}\left(f_{1}, \ldots, f_{6}\right)^{\top}= & \left(\kappa_{8} x_{2}-\frac{\kappa_{1} \kappa_{3}}{\kappa_{2}+\kappa_{3}} x_{1} x_{5},-\kappa_{4} x_{2} x_{5}+\kappa_{7} x_{3}-\kappa_{8} x_{2}+\frac{\kappa_{1} \kappa_{3}}{\kappa_{2}+\kappa_{3}} x_{1} x_{5},\right. \\
& \left.f_{3}, f_{4}, 0,-\frac{\kappa_{1}}{\kappa_{2}+\kappa_{3}} x_{1} x_{5}+x_{6}\right)^{\top}=\left(\tilde{f}_{1}, \ldots, \tilde{f}_{5}, \tilde{h}_{6}\right) .
\end{aligned}
$$

The corresponding digraph $G_{2}^{\circ}$ defined in 55.2 is:

$$
\mathrm{S}_{0} \underset{\kappa_{8}}{\stackrel{\frac{\kappa_{1}}{\kappa_{2}+\kappa_{3}} \times 5}{\rightleftharpoons}} \mathrm{S}_{1} \underset{\kappa_{7}}{\stackrel{\kappa_{4} \mathrm{x}_{5}}{\rightleftharpoons}} \mathrm{S}_{2} \underset{\kappa_{6}}{\stackrel{\kappa_{5} \mathrm{x}_{5}}{\rightleftharpoons}} \mathrm{S}_{3}
$$

and the underlying undirected graph is a tree with leaves $S_{0}$ and $S_{3}$. Then $\tilde{f}_{1}$ and $\tilde{f}_{4}$ are already binomials, and $p_{2}=\tilde{f}_{2}-\tilde{f}_{1}=-\kappa_{4} x_{2} x_{5}+\kappa_{7} x_{3}$ (from (37)), and $p_{3}=\tilde{f}_{3}+\tilde{f}_{4}=\left(\kappa_{4} x_{2} x_{5}-\kappa_{5} x_{3} x_{5}+\right.$ $\left.\kappa_{6} x_{4}-\kappa_{7} x_{3}\right)+\left(\kappa_{5} x_{3} x_{5}-\kappa_{6} x_{4}\right)=\kappa_{4} x_{2} x_{5}-\kappa_{7} x_{3}$, which are binomials. The matrix $M_{2}$ is

$$
M_{2}=\left(\begin{array}{cccccc}
1 & 0 & 0 & 0 & 0 & 0 \\
1 & 1 & 0 & 0 & 0 & 0 \\
0 & 0 & 1 & 1 & 0 & 0 \\
0 & 0 & 0 & 1 & 0 & 0 \\
0 & 0 & 0 & 0 & 1 & 0 \\
0 & 0 & 0 & 0 & 0 & 1
\end{array}\right)
$$


and $M_{2}\left(\tilde{f}_{1}, \ldots, \tilde{f}_{5}, \tilde{h}_{6}\right)^{\top}=\left(\tilde{h}_{1}, \ldots, \tilde{h}_{6}\right)^{\top}$.

In order to obtain $h_{c, \kappa, 2}, h_{c, \kappa, 3}, h_{c, \kappa, 4}, h_{c, \kappa, 6}$ we need to build $f_{1}$ and $f_{5}$ from $f_{c, \kappa, 2}, f_{c, \kappa, 3}, f_{c, \kappa, 4}$, $f_{c, \kappa, 6}$, multiply by the product $M_{2} M_{1}$, and then pick 4 linearly independent binomials:

$$
M^{\prime}=\left(\begin{array}{llllll}
1 & 0 & 0 & 0 & 0 & 0 \\
0 & 1 & 0 & 0 & 0 & 0 \\
0 & 0 & 0 & 1 & 0 & 0 \\
0 & 0 & 0 & 0 & 0 & 1
\end{array}\right) M_{2} M_{1}\left(\begin{array}{rrrr}
-1 & -1 & -1 & -1 \\
1 & 0 & 0 & 0 \\
0 & 1 & 0 & 0 \\
0 & 0 & 1 & 0 \\
0 & 0 & 0 & -1 \\
0 & 0 & 0 & 1
\end{array}\right)=\left(\begin{array}{rrrr}
-1 & -1 & -1 & -\frac{\kappa_{3}}{\kappa_{2}+\kappa_{3}} \\
0 & -1 & -1 & 0 \\
0 & 0 & 1 & 0 \\
0 & 0 & 0 & -\frac{1}{\kappa_{2}+\kappa_{3}}
\end{array}\right),
$$

which leads to $M^{\prime}\left(f_{c, \kappa, 2}, f_{c, \kappa, 3}, f_{c, \kappa, 4}, f_{c, \kappa, 6}\right)^{\top}=\left(\kappa_{8} x_{2}-\frac{\kappa_{1} \kappa_{3}}{\kappa_{2}+\kappa_{3}} x_{1} x_{5},-\kappa_{4} x_{2} x_{5}+\kappa_{7} x_{3}, \kappa_{5} x_{3} x_{5}-\right.$ $\left.\kappa_{6} x_{4},-\frac{\kappa_{1}}{\kappa_{2}+\kappa_{3}} x_{1} x_{5}+x_{6}\right)^{\top}$. Notice that, as $M_{1}(\kappa)$ is well defined for all $\kappa \in \mathbb{R}_{>0}^{8}$, then $M^{\prime}$ is also well defined for all $\kappa \in \mathbb{R}_{>0}^{8}$.

We now choose the matrix $M=M(\kappa)$ :

$$
M=\left(\begin{array}{rrrr}
\frac{1}{\kappa_{8}} & 0 & 0 & 0 \\
0 & \frac{1}{\kappa_{7}} & 0 & 0 \\
0 & 0 & -\frac{1}{\kappa_{6}} & 0 \\
0 & 0 & 0 & 1
\end{array}\right) M^{\prime}
$$

and the effective parameters $\bar{a}_{1}=\frac{\kappa_{1} \kappa_{3}}{\kappa_{8}\left(\kappa_{2}+\kappa_{3}\right)}, \bar{a}_{2}=\frac{\kappa_{4}}{\kappa_{7}}, \bar{a}_{3}=\frac{\kappa_{5}}{\kappa_{6}}$, and $\bar{a}_{4}=\frac{\kappa_{1}}{\kappa_{2}+\kappa_{3}}$. Then, the reparametrization map $\bar{a}$ is surjective, $M(\kappa)$ is well defined for all $\kappa \in \mathbb{R}_{>0}^{8}$, and $\operatorname{det}(M)=$ $\left(-\frac{1}{\kappa_{6} \kappa_{7} \kappa_{8}}\right) \cdot\left(-\frac{1}{\kappa_{2}+\kappa_{3}}\right)>0$.

\section{Appendix B. Proof of Lemma 6.4}

In this appendix, we prove Lemma 6.4.

Proof of Lemma 6.4. Let $\varepsilon>0$. By hypothesis, $f(a, z)$ and $\left(a^{*}, z^{*}\right) \in \mathbb{R}^{n+1}$ satisfy:

(I) $f\left(a^{*}, z^{*}\right)=0$

(II) $\frac{\partial f}{\partial z}\left(a^{*}, z^{*}\right)=0$,

(III) $\frac{\partial^{2} f}{\partial z^{2}}\left(a^{*}, z^{*}\right) \neq 0$, and

(IV) $\frac{\partial f}{\partial a_{\ell}}\left(a^{*}, z^{*}\right) \neq 0$.

By assumptions (I) and (IV), the implicit function theorem applies. So, there exists a function

$$
\tilde{\beta}: B_{\varepsilon^{\prime}}\left(a_{1}, \ldots, a_{\ell-1}, a_{\ell+1}, \ldots, a_{n}, z\right) \rightarrow \mathbb{R}
$$

defined on a ball of some radius $\varepsilon^{\prime} \leq \varepsilon$ in $\mathbb{R}^{n}$ such that $\tilde{\beta}\left(a_{1}^{*}, \ldots, a_{\ell-1}^{*}, a_{\ell+1}^{*}, \ldots, a_{n}^{*}, z^{*}\right)=a_{\ell}^{*}$ and, near the point of interest $\left(a^{*}, z^{*}\right)$, the $f=0$ locus is the graph of $\tilde{\beta}$.

Call $\beta:\left(z^{*}-\epsilon^{\prime}, z^{*}+\epsilon^{\prime}\right) \rightarrow \mathbb{R}$ the restriction $\beta(z)=\tilde{\beta}\left(a_{1}^{*}, \ldots, a_{\ell-1}^{*}, a_{\ell+1}^{*}, \ldots, a_{n}^{*}, z\right)$, and call $\hat{\beta}(z)=\left(a_{1}^{*}, \ldots, a_{l-1}^{*}, \beta(z), a_{\ell+1}^{*}, \ldots, a_{n}^{*}\right)$; then near $\left(a^{*}, z^{*}\right)$ we have

$$
f(\hat{\beta}(z), z)=0 \quad \text { for } z \in\left(z^{*}-\varepsilon^{\prime}, z^{*}+\varepsilon^{\prime}\right) .
$$

Take the derivative of equation (38), via the chain rule:

$$
\frac{\partial f}{\partial a_{\ell}}(\hat{\beta}(z), z) \beta^{\prime}(z)+\frac{\partial f}{\partial z}(\hat{\beta}(z), z)=0 .
$$


Evaluating this equation at $z=z^{*}$, and recalling that $\beta\left(z^{*}\right)=a_{\ell}^{*}$ and $\hat{\beta}\left(z^{*}\right)=a^{*}$, we obtain:

$$
\frac{\partial f}{\partial a_{\ell}}\left(a^{*}, z^{*}\right) \beta^{\prime}\left(z^{*}\right)+\frac{\partial f}{\partial z}\left(a^{*}, z^{*}\right)=0 .
$$

Recall that $\frac{\partial f}{\partial a_{\ell}}\left(a^{*}, z^{*}\right) \neq 0$, by hypothesis (IV), and $\frac{\partial f}{\partial z}\left(a^{*}, z^{*}\right)=0$, by (II). Thus, $\beta^{\prime}\left(z^{*}\right)=0$. Next, take another derivative, applying the chain rule to equation (39), and then evaluate at $z=z^{*}$ :

$$
\frac{\partial f}{\partial a_{\ell}}\left(a^{*}, z^{*}\right) \beta^{\prime \prime}\left(z^{*}\right)+\frac{\partial^{2} f}{\partial a_{\ell}^{2}}\left(a^{*}, z^{*}\right)\left(\beta^{\prime}\left(z^{*}\right)\right)^{2}+2 \frac{\partial^{2} f}{\partial z \partial a_{\ell}}\left(a^{*}, z^{*}\right) \beta^{\prime}\left(z^{*}\right)+\frac{\partial^{2} f}{\partial z^{2}}\left(a^{*}, z^{*}\right)=0 .
$$

Thus, we deduce from (III) and (IV) that $\beta^{\prime \prime}\left(z^{*}\right) \neq 0$. It follows that the univariate function $\beta$ has a maximum or a minimum at $z^{*}$ (depending on the sign of the second derivative). Hence, there exists a sufficiently small $\delta>0$ such that for all $\delta^{\prime} \in(0, \delta)$, either $b_{\ell}^{* *}=a_{\ell}^{*}-\delta^{\prime}$ (if $a_{\ell}^{*}$ is a local maximum) or $a_{\ell}^{* *}=a_{\ell}^{*}+\delta^{\prime}$ (if $a_{\ell}^{*}$ is a local minimum) yields $f\left(a^{* *}, z\right)=0$, for $a^{* *}=\left(a_{1}^{*}, \ldots, a_{\ell-1}^{*}, a_{\ell}^{* *}, a_{\ell+1}^{*}, \ldots, a_{n}^{*}\right)$, with two distinct real solutions within distance $\varepsilon$ of $z^{*}$.

\section{Appendix C. Proof of Theorem 6.11}

The goal of this appendix is to prove Theorem 6.11. We first recall some definitions and a result of Kapur, Sun, and Wang from the theory of comprehensive Gröbner bases [29]. For basic concepts from computational algebraic geometry, see the books [6, 7].

Let $h \in \mathbb{C}[a, x]:=\mathbb{C}\left[a_{1}, a_{2}, \ldots, a_{n}, x_{1}, x_{2}, \ldots, x_{s}\right]$. We denote by

$$
\operatorname{lpp}_{x}(h) \quad \text { and } \quad \operatorname{lc}_{x}(h),
$$

the leading monomial (or "leading power product") and leading coefficient of $h$, respectively, when $h$ is viewed in $\mathbb{C}(a)[x]$ taken with the lexicographic order $x_{s}<\cdots<x_{2}<x_{1}$. For instance, if $h=a_{1}^{2} x_{1}+x_{2}$, then $\operatorname{lpp}_{x}(h)=x_{1}$ and $\operatorname{lc}_{x}(h)=a_{1}^{2}$.

Definition C.1. [29, Definition 4.1] Given $H \subseteq \mathbb{C}[a, x]$, a subset $H^{\prime}$ of $H$ is a noncomparable subset of $H$ if

(1) for every $h \in H$, there exists $g \in H^{\prime}$ such that $\operatorname{lpp}_{x}(h)$ is a multiple of $\operatorname{lpp}_{x}(g)$, and

(2) for every $g_{1}, g_{2} \in H^{\prime}$, with $g_{1} \neq g_{2}$, the leading monomial $\operatorname{lpp}_{x}\left(g_{1}\right)$ is not a multiple of $\operatorname{lpp}_{x}\left(g_{2}\right)$, and $\operatorname{lpp}_{x}\left(g_{2}\right)$ is not a multiple of $\operatorname{lpp}_{x}\left(g_{1}\right)$.

Example C.2. Consider $H=\left\{a_{2} x_{2}^{2}-1, a_{1} x_{1}-1,\left(a_{1}+1\right) x_{1}-x_{2},\left(a_{1}+1\right) x_{2}-a_{1}\right\}$. Let $H^{\prime}=$ $\left\{a_{1} x_{1}-1,\left(a_{1}+1\right) x_{2}-a_{1}\right\}(\subseteq H)$. We verify that $H^{\prime}$ is a noncomparable subset of $H$ :

(1) Note that $\left\{\operatorname{lpp}_{x}(h) \mid h \in H\right\}=\left\{x_{2}^{2}, x_{2}, x_{1}\right\}$ and $\left\{\operatorname{lpp}_{x}(g) \mid g \in H^{\prime}\right\}=\left\{x_{2}, x_{1}\right\}$. So, every monomial in the first set is a multiple of some monomial in the second set.

(2) For the two polynomials in $H^{\prime}$, their leading monomials are, respectively, $x_{1}$ and $x_{2}$. We see that $x_{1}$ is not a multiple of $x_{2}$, and $x_{2}$ is not a multiple of $x_{1}$.

The following straightforward lemma shows that noncomparable subsets always exist and explains how (in theory) to effectively find one.

Lemma C.3 (Existence of noncomparable subsets). Let $H$ be a finite, nonempty subset of $\mathbb{C}[a, x]$. The following procedure yields a noncomparable subset of $H$ :

(1) Let $M=\left\{\operatorname{lpp}_{x}(h) \mid h \in H\right\}$, and let $D=\emptyset$. 
(2) Pick a monomial from $M$, say $m_{1}$. Let $d:=m_{1}$. Search $M$. If there exists $m \in M$ such that $m \mid d$ and $m \neq d$, then set $d:=m$. Continue searching until there is no $m$ in $M$ such that $m \mid d$ and $m \neq d$. Then add $d$ into $D$.

(3) Let $M^{\prime}:=\{m \in M: d \mid m\}$, and let $M:=M \backslash M^{\prime}$.

(4) Repeat steps 2-3 until $M$ is empty.

(5) For each $d \in D$, pick some $f_{d} \in F$ for which $\operatorname{lpp}_{x}(f)=d$. Output $F_{D}:=\left\{f_{d} \mid d \in D\right\}$.

Now consider a set of $s$ polynomials $H=\left\{h_{1}, h_{2}, \ldots, h_{s}\right\}$ in $\mathbb{C}[a, x]$. Denote by $\mathcal{I}(H)$ the ideal generated by $H$ in the polynomial ring. Denote by $V(H)$ the variety generated by $H$ (or, equivalently generated by $\mathcal{I}(H))$ in $\mathbb{C}^{n+s}$ :

$$
V(H):=\left\{(a ; x)=\left(a_{1}, a_{2}, \ldots a_{n}, x_{1}, x_{2}, \ldots, x_{s}\right) \in \mathbb{C}^{n+s} \mid h(a ; x)=0 \text { for all } h \in \mathcal{I}(H)\right\} .
$$

Below, we show that if the system $H$ is a general zero-dimensional system (Definition 6.7), then $H$ admits a triangular form (see the proof of Theorem 6.11). More specifically, we prove that any noncomparable subset of a Gröbner basis of $\mathcal{I}(H)$ with respect to the lexicographic order $a_{n}<\cdots<a_{2}<a_{1}<x_{s}<\cdots<x_{2}<x_{1}$ has the desired triangular form. The proof requires the following result, due to Kapur, Sun, and Wang [29, Theorem 4.3], which relates Gröbner bases of $\mathcal{I}(H)$ to those of the specialized ideal $\mathcal{I}\left(\left.H\right|_{b=b^{*}}\right)$ :

Proposition C.4 (Specialization of Gröbner bases [29]). Consider $H \subseteq \mathbb{C}[a, x]$, and let $\mathcal{G}$ be $a$ Gröbner basis of the ideal $\mathcal{I}(H) \subseteq \mathbb{C}[a, x]$ with respect to the lexicographic order $a_{n}<\cdots<a_{2}<$ $a_{1}<x_{s}<\cdots<x_{2}<x_{1}$. Let $\mathcal{G}_{\cap}=\mathcal{G} \cap \mathbb{C}[a]$, let $\mathcal{G}_{m}$ be a noncomparable subset of $\mathcal{G} \backslash \mathcal{G}_{\cap}$, and let $h=\Pi_{g \in \mathcal{G}_{m}} \mathrm{lc}_{x}(g)$. For any $a^{*}=\left(a_{1}^{*}, a_{1}^{*}, \ldots, a_{n}^{*}\right) \in \mathbb{C}^{n}$, if $\left.\mathcal{G}_{\cap}\right|_{a=a^{*}} \subseteq\{0\}$ and $\left.h\right|_{a=a^{*}} \neq 0$, then $\left.\mathcal{G}_{m}\right|_{a=a^{*}}$ is a Gröbner basis of the ideal $\mathcal{I}\left(\left.H\right|_{a=a^{*}}\right) \subseteq \mathbb{C}[x]$ with respect to the lexicographic order $x_{s}<\cdots<x_{2}<x_{1}$.

To prove Theorem 6.11, we also need the following lemma:

Lemma C.5. For a general zero-dimensional system $H=\left\{h_{1}, h_{2}, \ldots, h_{s}\right\} \subseteq \mathbb{C}[a, x]$, we have $\mathcal{I}(H) \cap \mathbb{C}[a]=\{0\}$.

Proof. Since $H$ is a general zero-dimensional system (Definition 6.7), there exists a proper variety $\mathcal{W} \subsetneq \mathbb{C}^{n}$ such that for every $a^{*} \in \mathbb{C}^{n} \backslash \mathcal{W}$ the specialized system $\left.H\right|_{a=a^{*}}$ has at least one complex solution. It follows that $\mathbb{C}^{n} \backslash \mathcal{W} \subseteq \pi(V(H))$, where $\pi: \mathbb{C}^{n+s} \rightarrow \mathbb{C}^{n}$ denotes the standard projection given by $(a, x) \mapsto a$. Thus,

$$
\overline{\mathbb{C}^{n} \backslash \mathcal{W}} \subseteq \overline{\pi(V(H))} \subseteq \mathbb{C}^{n} .
$$

Note that $\overline{\mathbb{C}^{n} \backslash \mathcal{W}}=\mathbb{C}^{n}\left(\right.$ as $\left.\mathcal{W} \subsetneq \mathbb{C}^{n}\right)$, so $\overline{\pi(V(H)}=\mathbb{C}^{n}$. By [7, pg. 193, Thm. 3], we know that $\overline{\pi(V(H))}=V(\mathcal{I}(H) \cap \mathbb{C}[a])$. So, $V(\mathcal{I}(H) \cap \mathbb{C}[a])=\mathbb{C}^{n}$. The only ideal that generates the variety $\mathbb{C}^{n}$ is the zero ideal. So, $\mathcal{I}(H) \cap \mathbb{C}[a]=\{0\}$.

Proof of Theorem 6.11. Let $H=\left\{h_{1}, h_{2}, \ldots, h_{s}\right\}$. Let $\mathcal{G}_{m}$ be a noncomparable subset of $\mathcal{G}$ (which exists by Lemma C.3). By Lemma C.5, we have:

$$
\mathcal{G}_{m} \cap \mathbb{C}[a] \subseteq \mathcal{I}(H) \cap \mathbb{C}[a]=\{0\} .
$$

In fact, $0 \notin \mathcal{G}_{m}$ (by the definition of noncomparable subset and because $\mathcal{G} \neq\{0\}$ ), so $\mathcal{G}_{m} \cap \mathbb{C}[a]=\emptyset$. So, by Proposition C.4 for every $a^{*} \in \mathbb{C}^{n} \backslash V(h)$, where $h=\Pi_{g \in \mathcal{G}_{m}} \mathrm{lc}_{x}(g)$, the set $\left.\mathcal{G}_{m}\right|_{a=a^{*}}$ is a Gröbner basis of $\mathcal{I}\left(\left.H\right|_{a=a^{*}}\right) \subseteq \mathbb{C}[x]$ with respect to $x_{s}<\cdots<x_{1}$. 
(1) We show that a subset $\left\{g_{1}, g_{2}, \ldots, g_{s}\right\}$ of $\mathcal{G}_{m}$ has the required triangular form. As $H$ is a general zero-dimensional system, let $\mathcal{W}$ be the variety in $\mathbb{C}^{n}$ such that $H$ satisfies the hypotheses (A1)-(A3) in Definition 6.7. Let $c^{*} \in \mathbb{C}^{n} \backslash(\mathcal{W} \cup V(h))$. Then by (A1) in Definition 6.7, we know that $V\left(\left.\mathcal{G}_{m}\right|_{a=c^{*}}\right)=V\left(\left.H\right|_{a=c^{*}}\right)$ is a nonempty finite set in $\mathbb{C}^{s}$. Hence, by [7, pg. 234, Thm. 6 (i) and (iii)], for $i \in\{1,2, \ldots, s\}$, there exists $g_{i} \in \mathcal{G}_{m}$ such that the leading monomial of $\left.g_{i}\right|_{a=c^{*}}$ has the form $x_{i}^{N_{i}}$, where $N_{i}$ is a non-negative integer. In particular, $\left.g_{s}\right|_{a=c^{*}} \in \mathbb{C}\left[x_{s}\right]$.

It follows that $\operatorname{lpp}_{x}\left(g_{i}\right)=x_{i}^{N_{i}}$, because $c^{*} \notin V(h)$ and so $\left.\operatorname{lc}_{x}\left(g_{i}\right)\right|_{a=c^{*}} \neq 0$. Hence, if we show that $N_{2}=N_{3}=\cdots=N_{s}=1$, then, by the definition of the lexicographic order, $g_{1}, g_{2}, \ldots, g_{s}$ have the forms shown in Theorem 6.11 (1).

Hence, to finish proving (1), we need only show that $N_{2}=N_{3}=\cdots=N_{s}=1$. Let $N:=$ $\left|V\left(\left.H\right|_{a=c^{*}}\right)\right|$. Then every $x^{*} \in V\left(\left.H\right|_{a=c^{*}}\right)$ has a distinct $x_{s^{-}}$-coordinate (by (A2) in Definition 6.7), and every such coordinate is a root of $\left.g_{s}\right|_{a=c^{*}} \in \mathbb{C}\left[x_{s}\right]$. Hence,

$$
N_{s}=\operatorname{deg}\left(\left.g_{s}\right|_{a=c^{*}}\right) \geq N \text {. }
$$

Next, by (A3) in Definition 6.7 and [7, pg. 235, Prop. 8(ii)], we know that

$$
N=N_{1} N_{2} \cdots N_{s} \text {. }
$$

So, by (40) and (41), we have $N_{s}=N$ and $N_{2}=N_{3}=\cdots=N_{s-1}=1$.

(2) Consider the following claim:

Claim: $\mathcal{G}_{m}=\left\{g_{1}, g_{2}, \ldots, g_{s}\right\}$.

This claim implies that $h=Q_{s, N} Q_{1} Q_{2} \cdots Q_{s-1}$ and so, by what we saw earlier, for every $a^{*} \in$ $\mathbb{C}^{n} \backslash V\left(Q_{s, N} Q_{1} Q_{2} \cdots Q_{s-1}\right)$, the set $\left\{\left.g_{1}\right|_{a=a^{*}},\left.g_{2}\right|_{a=a^{*}}, \ldots,\left.g_{s}\right|_{a=a^{*}}\right\}$ is a Gröbner basis of the ideal $\mathcal{I}\left(\left.H\right|_{a=a^{*}}\right)$ with respect to $x_{s}<\cdots<x_{1}$. So, to complete the proof, we need only prove the Claim.

First, the containment $\mathcal{G}_{m} \supseteq\left\{g_{1}, g_{2}, \ldots g_{s}\right\}$ follows from the fact that the $g_{i}$ 's were selected from $\mathcal{G}_{m}$. Next, we show the containment $\mathcal{G}_{m} \subseteq\left\{g_{1}, g_{2}, \ldots, g_{s}\right\}$ by proving the following equality by induction on $i$ :

$$
\mathcal{G}_{m} \cap \mathbb{C}\left[a, x_{i}, x_{i+1} \ldots, x_{s}\right] \subseteq\left\{g_{i}, g_{i+1}, \ldots, g_{s}\right\}, \quad \text { for all } i \in\{1,2, \ldots, s\} .
$$

For $i=s$, assume that $g \in \mathcal{G}_{m} \cap \mathbb{C}\left[a, x_{s}\right]$. Then $\operatorname{lpp}_{x}(g)=x_{s}^{\tilde{N}}$ for some $\tilde{N} \geq 0$, and also recall that $\operatorname{lpp}_{x}\left(g_{s}\right)=x_{s}^{N}$ for some $N>0$. However, $\mathcal{G}_{m}$ is noncomparable, so $g=g_{s}$.

For the inductive step, assume that the containment (42) holds for all $i \in\{j, j+1, \ldots, s\}$ (for some $j \leq s)$. For $i=j-1$, let $g \in \mathcal{G}_{m} \cap \mathbb{C}\left[a_{1}, a_{2}, \ldots, a_{n}, x_{j-1}, x_{j}, \ldots, x_{s}\right]$, and write $\operatorname{lpp}_{x}(g)=$ $x_{s}^{a_{s}} \cdots x_{j}^{a_{j}} x_{j-1}^{a_{j-1}}$. If $a_{j-1}=0$, then $g \in \mathcal{G}_{m} \cap \mathbb{C}\left[a_{1}, a_{2}, \ldots, a_{n}, x_{j}, x_{j+1}, \ldots, x_{s}\right]$, so by the induction hypothesis, $g \in\left\{g_{j}, g_{j+1}, \ldots, g_{s}\right\}$. If $a_{j-1}>0$, then $\operatorname{lpp}_{x}\left(g_{j-1}\right)=x_{j-1} \mid \operatorname{lpp}_{x}(g)$. Hence, $g=g_{j-1}$ (because $\mathcal{G}_{m}$ is noncomparable).

A. D. and M. P. M: Departamento de Matemática, FCEN, Universidad de Buenos Aires e IMAS (UBAConicet), Ciudad Universitaria, Pab.I, 1428 Buenos Aires, Argentina, A. S. and X. T.: Department of Mathematics, Texas A\&M University, College Station TX 77843, USA.

E-mail address: alidick@dm.uba.ar,mpmillan@dm.uba.ar, annejls@math.tamu.edu, xiaoxian@math.tamu.edu 